Portland State University

PDXScholar

6-20-1972

\title{
Complex Systems and the Price-Resource Directive Coordination Procedure
}

Chamberlain Lambros Foes

Portland State University

Follow this and additional works at: https://pdxscholar.library.pdx.edu/open_access_etds

Part of the Systems Science Commons

Let us know how access to this document benefits you.

\section{Recommended Citation}

Foes, Chamberlain Lambros, "Complex Systems and the Price-Resource Directive Coordination Procedure" (1972). Dissertations and Theses. Paper 831.

https://doi.org/10.15760/etd.831

This Dissertation is brought to you for free and open access. It has been accepted for inclusion in Dissertations and Theses by an authorized administrator of PDXScholar. Please contact us if we can make this document more accessible: pdxscholar@pdx.edu. 


\title{
COMPLEX SYSTEMS AND THE PRICE-RESOURCE \\ DIRECTIVE COORDINATION PROCEDURE
}

by

CHAMBERLAIN IAMBROS FOES

\author{
A thesis submitted in partial fulfillment of the \\ requirements for the degree of \\ DOCTOR OF PHILOSOPHY \\ in \\ SYSTEMS SCIENCE
}

\section{Portland State University 1972}

(C) 1972 Chamberlain Lambros Foes 
AN ABSTRACT OF THE PH.D. DISSERTATION OF Chamberlain Lambros Foes for the degree of Doctor of Philosophy in Systems Science presented June 20,1972 .

Title: Complex Systems and the Price-Resource Directive Coordination Procedure.

In this thesis, the problem considered is that of linear static optimization of a large system which is composed of a finite number of subsystems, each characterized by its own constraint matrix and objective function. The total system is itself constrained by resource availabilities and other factors, and its objective function is the mathematical linear sum of the objective function of the subsystems. The total system constraints couple together all the subsystems. The total system is first reformulated as a two-level problem by decoupling the total system constraints utilizing an arbitrary partition of the total system resources and other factors according to the number of subsystems. At the upper level we have a so-called central problem having as an objective function the sum of the optima of the subsystems achieved for any given partition and constrained by the total available resources and other factors which can be partitioned. At the lower level we have the subproblems which are small-dimension linear programming problems parameterized on the right-hand side of the part of the constraints which resulted from the decoupling of the total system constraints.

The resources vector of each subproblem's set of constraints contains the system common resources allocated to it by the central 
problem. Different allocations of these resources to each subsystem create multiparametric optimization problems for which we have solution methods. The subsystem solutions become functions of the central system allocation policies. Therefore, the major concern for optimization of the whole system is the discovery of the optimum allocation policy.

The method that we introduce finds the optimum allocation policy in a finite number of different allocation iterations. The major steps in the development are the discovery that the minimal (in case of multiple solutions) shadow prices of the subsystems are equal at optimality to the central system shadow prices, and that a coordination of the subsystems for the purpose of achieving optimality of the total system can be organized by utilizing the concave relationships governing the subsystem shadow prices versus the resources allocated to these subsystems.

The method offers significant computational and conceptual advantages over present decomposition techniques, since it disposes with the solution of a central problem and the subproblems at each iteration and substitutes instead a simple coordination operation and subsystem parametric optimization at each iteration after the first, where a full solution of the subsystems takes place. 


\section{ACKNOWLEDGMENTS}

The author wishes to thank his thesis supervisor and chairman of his dissertation committee Professor Abdul Qayum for his aid, encouragement and the long hours of consultations he provided during the course of this work. Appreciation is also extended to professors S. Tauber, H. White, H. Linstone and Vice President J. Blumel for their helpful suggestions.

The graduate education of the author was made possible by the generosity of professors $\mathrm{H}$. White and $\mathrm{H}$. Linstone who provided graduate teaching assistantships. 
TABLE OF CONTENTS

PAGE

ACKNOWLEDGMENTS . . . . . . . . . . . . . . . . ii

LIST OF TABLES . . . . . . . . . . . . . . . . . . v v

LIST OF FIGURES . . . . . . . . . . . . . . . . . . vi vi

CHAPTER

I INTRODUCTION .................... 1

II COMPLEX AND IARGE-SCALE SYSTEMS . . . . . . . . . 4

Characteristics of Large-Scale Systems . . . . 6

Taxonomy of Irarge-Scale Systems ........ 10

Static Regime Large-Scale System Problem . . . . 12

III PRICE-RESOURCE DIRECTIVE COORDINATION: BASIC THEORY • 19

The Block-Angular Structured Linear System . . . 20

Properties of the Function .......... 31

Optimality Conditions for the Central

System Problem ........... 47

The Saddle Point Problem and Optimality

Development of the Central Problem

Optimality Condition

IV PRICE-RESOURCE DIRECTIVE COORDINATION: THE ALGORITHM • 64

The Direction Finding Problem . . . . . . 68

Summary of the Procedure ........... 77 
CHAPTER

PAGE

V PRICE-RESOURCE DIRECTIVE COORDINATION: APPLICATION • . 80 Decentralized Optimal Economic Planning . . . . 80

Multifurnace Aluminum Alloy Blending . . . . . 86

REFERENCES .......................... . 89

APPENDIX A . . . . . . . . . . . . . . . . 95

APPENDIX B . . . . . . . . . . . . . . . 107 


\section{LIST OF TABLES}

I Classification of Systems in Order of Complexity . . . 6

II General Classification of All Systems . . . . . . . 11

III Taxonomy of Systems by Activity Level . . . . . . 12 


\section{LIST OF FIGURES}

FIGURE

PAGE

1 Subsystem Objective Function Variation with

Change in the Resources .......... . 41

2 Subsystem Shadow Price Variation with Change

in the Resources.............. 41

3 Calculation of Resource Reallocation . . . . . 76

4 General System with Interacting Elements ....... 81 
CHAPTER I

\section{INTRODUCTION}

The rational study, analysis, design, construction and control of complex systems is characteristic of modern science and technology in general and of systems science and operations research in particular. Significant progress towards the design of analysis and optimization methods for complex systems has been achieved in the last two decades, but the emergence of an axiomatic theory for complex systems and the subsequent standardization of solution approaches has not been accomplished as of yet (1). Serious efforts towards this direction were multicentered in the last ten years, but the results are still fragmentary $(13,14,15)$. Despite the progress in the analysis of complex systems, in the last few years only a very small part of the required solutions has been obtained and that centers around the notion of decomposition, which in its general sense will also be used here.

In this thesis, the problem considered is that of linear static optimization of a large system which is composed of a finite number of subsystems, each characterized by its own constraint matrix and objective function. The total system is itself constrained by resource availabilities and other factors, and its objective function is the mathematical linear sum of the objective function of the subsystems. The total system constraints couple together all the subsystems. 
The total system is first reformulated as a two-level problem by decoupling the total system constraints utilizing an arbitrary partition of the total system resources and other factors according to the number of subsystems. At the upper level we have a so-called central problem having as an objective function the sum of the optima of the subsystems achieved for any given partition and constrained by the total available resources and other factors which can be partitioned. At the lower level we have the subproblems which are smal1-dimension linear programming problems parameterized on the right-hand side of the part of the constraints which resulted from the decoupling of the total system constraints.

The resources vector of each subproblem's set of constraints contains the system common resources allocated to it by the central problem. Different allocations of these resources to each subsystem create multiparametric optimization problems for which we have solution methods. The subsystem solutions become functions of the central system allocation policies. Therefore, the major concern for optimization of the whole system is the discovery of the optimum allocation policy.

The method that we introduce finds the optimum allocation policy in a finite number of different allocation iterations.

The major steps in the development are the discovery that the minimal (in case of multiple solutions) shadow prices of the subsystems are equal at optimality to the central system shadow prices, and that a coordination of the subsystems for the purpose of achieving optimality 
of the total system can be organized by utilizing the concave relationships governing the subsystem shadow prices versus the resources allocated to these subsystems.

The method offers significant computational and conceptual advantages over present decomposition techniques, since it disposes with the solution of a central problem and the subproblems at each iteration and substitutes instead a simple coordination operation and subsystem parametric optimization at each iteration after the first, where a full solution of the subsystems takes place.

Before the new method is presented, an effort will be made to classify some of the definitional ambiguities and characteristics surrounding complex systems in a manner that will make it possible for future extensions of the procedure to be more complex system oriented. 
CHAPTER II

COMPLEX AND LARGE-SCALE SYSTEMS

Approximately, a system is defined to be a set of interrelated elements which must be thought of as forming a certain whole. In practical terms, systems are frequently encountered whose unity of elements manifests itself by certain interactions demonstrating purposeful behavior towards the attainment of specific goals. On the technological level an example of a system could be given all the way from the micro-level of a minox camera system to the macro-level of a petrochemical plant or a space shuttle system. On the social level an organization is a system and exhibits a rather more complex behavior due to the fact that this system contains human groups, which enlarges the possibility of contradiction between the system common goals and the local aims of the subsystems and the individual elements (2). The complexity of an organization becomes more intense when, in order to implement complex endeavors and mammoth programs, it becomes a polyorganization (14)--in other words, a loosely-connected conglomerate of organizations.

The subject of system complexity is an uncharted territory and only lately efforts are being made to characterize complexity according to certain vague criteria $(3,7,11,14,16,17,18)$. Nothing approaching standardization for the classification of complex systems 
exists. This complexity in the systems arises from the multiplicity of subsystems and elements, the diverse operations performed by them, the high order of interaction and interconnection of the elements, the large amount of information to be processed and among other attributes the great variety of possible forms of system controls $(17,18)$.

A system can also be defined by its structure and behavior (6). Usually, complexity of structure is accompanied by complexity of system behavior, and the structure complexity can be charactexized by the complexity of the system behavior, which is characterized by the types of system reaction to external disturbances.

In terms of increasing complexity of behavior, the different types of actual systems as given by Fleyshman (3) are summarized in Table I. According to Fleyshman's classification of systems, we have the following main definitions.

Definition 1.1. A Simple_System is a system that does not exceed automatic systems in complexity.

Definition 1.2. A Complex System is a system more complex than automatic systems.

Definition 1.3. A Large System is a large aggregation of complex systems with comparatively weak connections among them.

A formal and strict definition of a large system does not exist as yet, although the difference between simple systems and complex ones is revealed by their qualitative characteristics as will be shown in the next section. 
TABLE I

CLASSIFICATION OF SYSTEMS IN ORDER OF COMPLEXITY

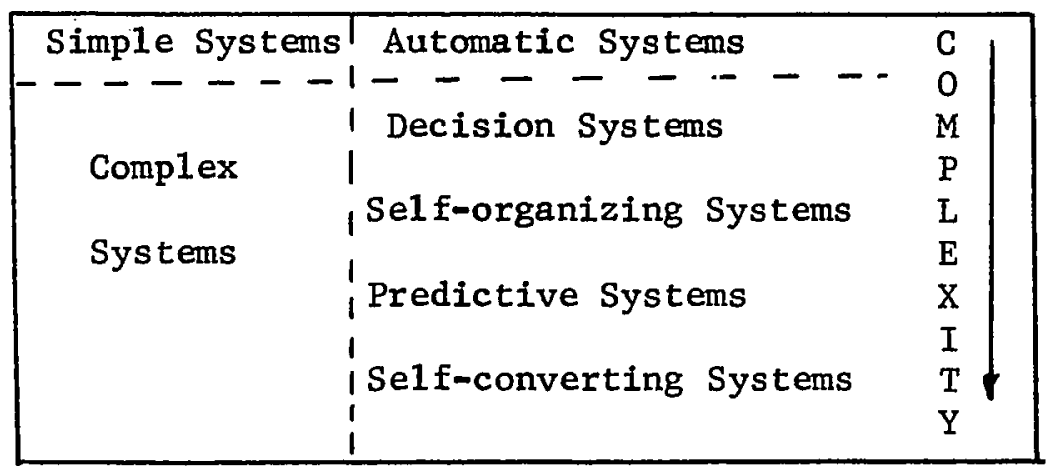

I. CHARACTERISTICS OF LARGE-SCALE SYSTEMS

In practical terms, a large-scale system is characterized by the presence of high dimensionality (4) determined by the number of variables or the number and complexity of structural equations describing the system or the complexity of the system performance criterion functional or a combination of part or all of the above. Usually, the factors that must be taken into account may number in the millions and classical operations research methods that may be quite tractable for thousands of variables run on their boundary of limitations when confronted with millions. System size has an exponential effect on the time needed for computation, so that entirely new approaches must be found for the solution of large-scale system problems. Some of the new approaches that have been developed, as are presented in the subsequent chapters of this study, utilize another major characteristic of large-scale systems, their hierarchical structure.

A number of authors agree that a hierarchical structure is the most characteristic feature of large-scale systems (7, p. 59), and in 
large organizations a hierarchical chain of command is nearly always present (5). Hierarchy exists in large-scale systems because they are composed of higher forms of complex systems which are capable of autonomous action and, in practice, delegation of authority and control by the highest force in the system ensures system harmony. If there is no central force in the large-scale system, dissolution will result and the subsequent creation of independent systems might set the stage for cross-purpose behavior of these systems in which case the overall objective of the large-scale system cannot be accomplished.

The presence of hierarchy does not imply the absence of some conflict of interest between the higher and lower levels of the largescale system (9). On the contrary, the mere presence of hierarchy creates interleve1 conflict which suboptimizes the performance behavior of the whole system. Subsequently, the minimization of this interleve1 conflict by some method of coordination of the different levels could bring about an optimization of the performance criterion for the total large-scale system. This is the crux of the methods of large-scale mathematical optimization.

Essentially, in cybernetic terminology (10), a method of coordination can be found by the establishment of a central governor and subsystem subgovernors which continuously can compare some behavioral aspects of the large-scale system against a standard and then engage in automatic feedback of corrective action to bring about a continuous optimization of the large-scale system performance criterion. If such a coordination method can be found, the large-scale system will become 
self-regulating within its physiological limits. In other words, it will become a homeostatic system. The word ecosystem used to be homeostatic before the advent of strong interference by man.

At the present time, the goal of homeostatic man-made systems is far from being achieved, some of the reasons being the presence in large-scale systems of a number of othe $r$ complex characteristics. Openness, which is the high degree of interaction of the largescale system with the dynamic external environment, is a characteristic which might make the design and construction of a large-scale system obsolete, by the time of completion. This can occur because the environment itself undergoes changes during that time. Furthermore, the introduction of the system itself may change the environment.

Another important characteristic of large-scale systems is the non-deterministic behavior (11). This mainly occurs because of the incompleteness and imprecision of the original information about the structure, state, and behavior of the system, and the uncertainty of future events.

Present methods for the analysis of even very sma11-scale scochastic systems result in problems of very high dimensionality in order to account for alternative possible realizations of the imperfectly known parameters (20). Methods for the analysis of large-scale stochastic problems do not even exist.

Problems of dimensionality arise also out of the characteristic change in the course of time of large-scale systems. This occurs because of the need to replicate variables and constraints to account for the great number of time periods (21). 
From the practical standpoint, another important characteristic of large-scale systems is the lack of hard data. Especially in the socio-economic areas only the crudest data wist. Efforts to collect data for the analysis and design of socio-economic systems run the risk of influencing adversely the system environment. The classical example of "Project Camelot" in Chile stands out as a sad commentary of these efforts (22).

From the mathematical standpoint, large-scale systems are characterized by nonlinearity and discontinuity in their interactions (12). Most modern methods for the analysis of complex systems assume monotonicity, continuity, convexity or other well-behaved characteristics for the performance functional and the system structure, and actually the most workable algorithms are to be found for linear systems.

Another disturbing characteristic of large-scale systems is the high degree of interdependence between subsystems in the same leve1.

In a rudimentary form, all these large-scale system characteristics are present even for simple systems. Examples are the hierarchical structure of atoms, the stochastic existence of an electron, and the changeability of bodies, associated with their formation and aging. However, all these properties appear in complex systems in a qualitatively new and more intensive form. For example, from the comparison of a simple system--a stone--and a complex one--a man--we can see in the latter case the openness being associated with the exchange of materials and ideas and the stochasticity being associated with complex psychic behavior. 


\section{TAXONOMY OF IARGE-SCALE SYSTEMS}

Diverse disciplines employ diverse classifications for the taxonomy of large-scale systems, but the classification which is general enough for all disciplines and should be enfranchised by them is the one due to cybernetic theory.

Cybernetic theory derived most of its postulates by diligent study of the behavior patterns of living organisms. Every stgtem was found capable of being characterized by its behavior pattern which regulates the system by imposing rules, giving learning mechanisms, etc. For practical purposes of modeling this behavior pattern divides the systems into two categories (24):

a) Isomorphic Systems

b) Homomorphio Systems

In brief, isomorphic systems are those that can be modeled element by element, in one-to-one mapping. Modeling that involves many-to-one transformations is called homomorphic. More precise definitions, in a mathematical form, are given by K1ir and Valach (24).

A good model of a system is a homomorph, because a homomorphic mapping preserves the structural relationships that are chosen to be preserved by the analyst, and this is enough for purposes of analysis and design.

A further classification implicit in cybernetic theory is given in Table II. 
TABLE II

GENERAL CLASSIFICATION OF ALL SYSTEMS

- Simple nonliving systems

- Simple living systems

- Comp1ex non1iving systems

- Complex living systems

The great concentration of effort has been a11 along in the analysis, design and optimization of complex nonliving systems.

It seems that extensive studies must be undertaken to correlate the characteristics of the complex living systems with those of the complex nonliving systems. This type of studies might be able to produce a body of basic theory that will give a better understanding for the solution of complex nonliving systems problems.

Another taxonomy of systems according to activity levels is given in Table IV. This table is based on (12).

The only classifications which have been standardized for largescale systems almost in the whole of technical 1iterature pertain to the optimization of large-scale systems.

The main definitional link between systems and their optimization is regime, and there are two regimes:

A. Static Regime system characterized by linear or nonlinear algebraic or transcendental equations describing it's structure and linear or nonlinear algebraic or transcendental functions describing its performance functiona1. Optimization of this static regime system is called Mathematical Programming. 
B. Dynamic Regime system characterized by linear or nonlinear ordinary or partial differential equations describing its structure and an integral describing its performance functional. Optimization of this dynamic regime system is called optimal control.

In this study the effort will be placed in the mathematical programing methods for large-scale systems. Methods for the optimal control of large-scale systems are to a great extent extensions of the mathematical programing methods as are implemented today, although methods particular to optimal control are continuously being developed (25).

TABLE III

TAXONOMY OF SYSTEMS BY ACTIVITY LEVEL

$\left.\begin{array}{|lll|}\hline \multicolumn{1}{|c|}{\text { Level }} & \multicolumn{2}{c|}{\text { System Description }} \\ \text { Pperational Systems } & \text { Sub-subsystems } & \text { Single Goal } \\ \text { Tactical Systems } & \text { Subsystems } & \text { Single Goal }\end{array}\right]$ Microsystems

III. THE STATIC REGIME IARGE-SCALE SYSTEM PROBLEM

"From its very inception, it was envisioned that linear programming would be applied to very large, detailed models of economic and military systems" (26). This statement by Dantzig in 1967 shows that after the War the analysts became aware that the piecemeal approach to the solution of operations research problems was basically an exercise in sub- 
optimization. They realized that the optimization of each subsystem did not produce an optimal system. Of course, the fundamental assumption underlying their approach was linearity, which can only be approximated for large-scale systems. The fact remains though that up to the present time most of the socio-economic and technical systems have been studied under the linearity assumption. Therefore, it is much easier to study the optimization of large-scale linear systems, where a wealth of information exists, than plunge into the unknown world of non-linearity, discontinuity and stockasticity.

The decision to work first on linear systems was a mixed blessing. On one hand great progress was achieved in the optimization of largescale deterministic linear systems. On the other hand, the analysts lost themselves working in wel1-ordered systems to be applied in a not too well-ordered world. In view of the present accumulating society problems with all their implications as complex systems, Alice of Wonderland fame found a home in the modern analysts laboratories. But then, Alice also visited George Boole's home in his time. The above remarks were made to emphasize that all the methods for large-scale optimization developed so far are nothing but a drop in the ocean of the required methods yet to come to enable real solution to complex systems problems. This will become more clear in the course of studying the various assumptions made for the development of the presert large-scale system optimization methods.

In later chapters, a new method is presented for the solution of the large-scale problem. This too is pervaded with assumptions as all the other methods are. The main point is that as long as there is deep 
understanding of the processes dominating complex systems gradually

a way will be found to lift one by one all these assumptions.

The static regime large-scale system optimization problem can be given as:

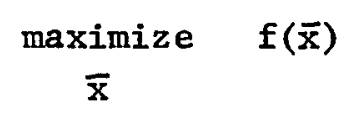

subject to

$$
\overline{\mathrm{x}} \in \mathrm{S}
$$

where

$$
\begin{aligned}
& s=S \cap s_{1} \cap \ldots \cap s_{m} \\
& s_{0} \subseteq R^{n} \\
& s_{i}=\left\{\bar{x} \mid g_{i}(\bar{x}) \leqslant b_{i}, i=1, \ldots, r\right\} \\
& s_{i}=\left\{\bar{x} \mid g_{i}(\bar{x})=b_{i}, i=r+1, \ldots, m\right\} \\
& \bar{x}=n-v e c t o r \text { of real variables } \\
& f(\bar{x})=\text { real-valued function defined on } s_{0} \\
& g_{i}(\bar{x})=r e a l-v a l u e d \text { function defined on } s_{0}
\end{aligned}
$$


The function $f(\bar{x})$ is the system performance criterion function, usually called the objective function. In engineering design problems the function $f(\bar{x})$ is the one which indicates the "best" design to be chosen (27), in economic planning it might be among a number of things, a welfare function or a preference function (28), in business it might be a profit or cost function (29). In general, the objective function can be given many interpretations and the design of a "good" objective function is a problem by itself (30).

The sets $s_{i}$ for $i=0, i, \ldots, m$ are the feasibility regions from which the objective function receives its vector $\vec{x}$. These feasibility regions are constructed by the function inequalities and equalities $g_{i}(\bar{x}) \leqslant 0$ for $i=1, \ldots, r$ and $g_{i}(\bar{x})=0$ for $i=r+1, \ldots, m$. These functions represent the structure of the system with its inputs and limitations. The vector $\bar{x}$ is the vector of the sought intensities of activity which will maximize the objective function. Of course, instead of maximization, minimization can be sought since:

$$
\underset{\bar{x}}{\operatorname{maximize}} \mathrm{f}(\overline{\mathrm{x}})=\underset{\bar{x}}{\operatorname{minimize}}-\mathrm{f}(\overline{\mathrm{x}})
$$

The basic assumption underlying the solution approaches for the large-scale static system $(1.1)-(1.2)$ is that the functions $f(\bar{x})$, $g_{i}(\bar{x}), i=1, \ldots, m$ are additively separable. In other words, problem $(1.1)-(1.2)$ can be written as:

maximize

$$
\sum_{l=1}^{\operatorname{lik}} f_{l}\left(\bar{x}_{l}\right)
$$


subject to

$$
\bar{x}_{e} \in s_{e}, \ell=1, \ldots, k
$$

where:

$$
\begin{aligned}
& s_{l}=s_{o} \cap s_{l i} \\
& s_{0} \subseteq R^{n} \\
& s_{\ell i}=\left\{\bar{x}_{e} \mid g_{e i}\left(\bar{x}_{l}\right) \leqslant b_{l i}, i=1, \ldots, r\right\} \\
& s_{\ell i}=\left\{\bar{x}_{e} \mid g_{e i}\left(\bar{x}_{l}\right)=b\right.
\end{aligned}
$$

If in reality the problem (1.1)-(1.2) can be written as problem (1.3)-(1.4) then the large-scale system can be considered completely separable in which case its solution can be simply achieved by solving $k$ different mathematical programming problems and suming their optimal solutions. But if the large-scale system is completely separable, meaning that there are no interlinking variables or constraints, it is not a system but a collection of independent systems.

As it was mentioned previously, a large-scale system has interdependent subsystems, in other words there are interlinking variables or coupling constraints which unite the large-scale system for a purposeful behavior. Therefore, the large-scale system is completely separable only in some of the constraints. The other "uniting" constraints are not additively separable because they unite and dominate the whole 
system. Therefore, problem (1.1)-(1.2) can be actually written in non-set notation and incorporating the two types of constraints into one as:

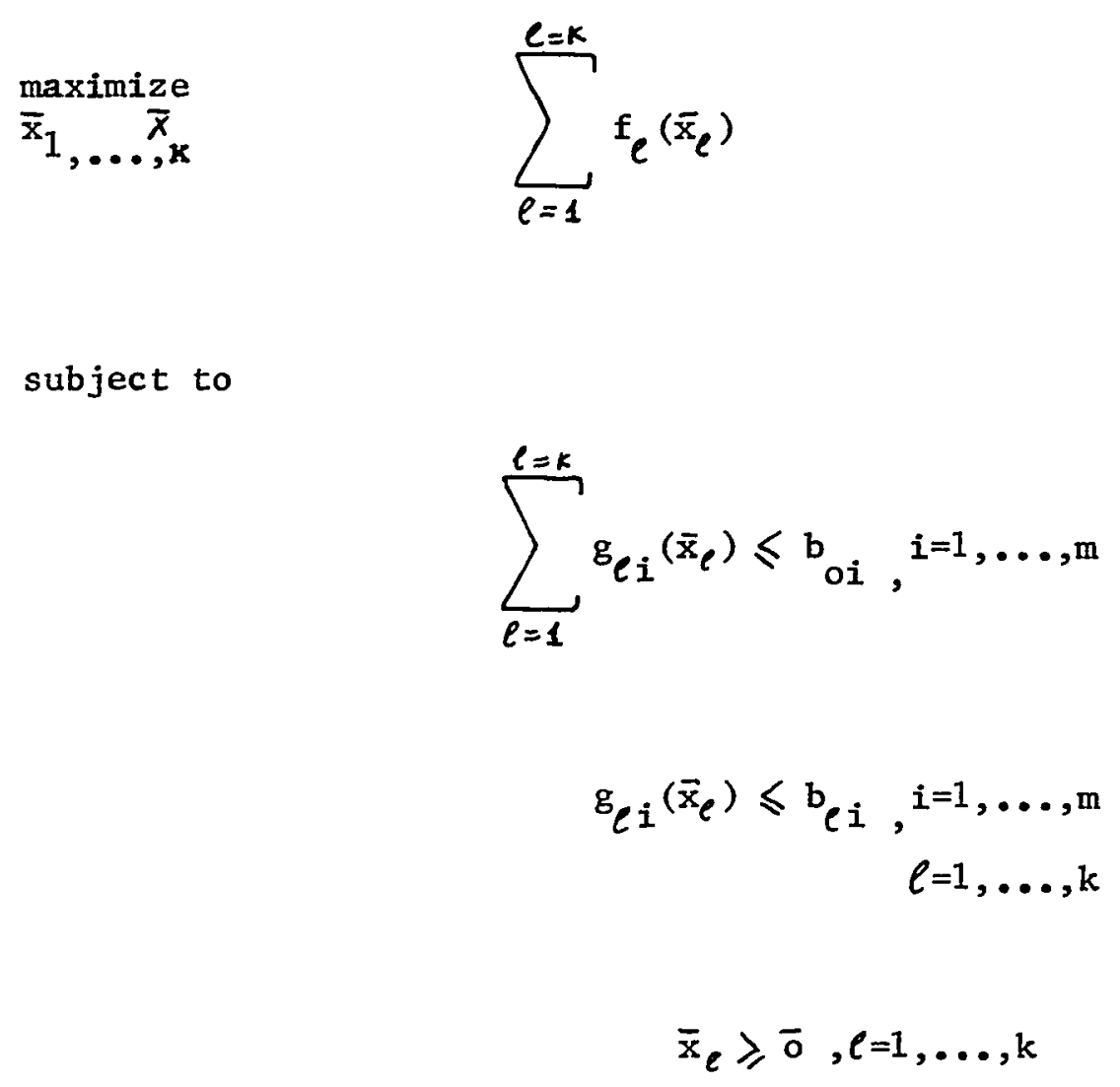

The assumption of additive separability is fundamental in the approaches for the solution of complex systems. The question that arises is that if really a complex system can be additively separated into subsystems. If it can, the implicetioñs axe that ine subsystein envelopes have been completely indentified and the large-scale system streams are al1 charted. That this cannot be true in real life systems in most cases could be proven by numerable examples. But, only through this assump ion progress has been made. To eliminate the separability 
assumption, completely novel optimization methods have to be developed and as of the present time there is not even a beginning in sight. Based on the additive separability principle two basic trends of development can be distinguished in the theory of large-scale systems optimization $(5,7,31)$. The first one is the analytic trend which attempts to decompose or break down the original large-scale problem into a number of independent simpler and smaller sub-problems, and simultaneously attempts to provide some means for coordinating them. Since a hierarchical structure is a basic characteristic of most large-scale systems the analytic trend is the one that provided most of the algorithons for optimization. Decomposition generates immediately a multi-level hierarchical structure and the exploitation of the properties of hierarchical structures provided the opening for the solution of the large-scale system problem.

The second trend is the synthetic trend which aims at aggregating a multiplicity of interconnected subsystems with known performance properties into a large system with the desired characteristics.

The methods of aggregation are not sufficiently developed and tested or formalized to permit serious comparative analysis and evaluations. Some procedures are given in Wismer (32), and some interesting work is presented by Ven and Erlike (33). 
CHAPTER III

PRICE-RESOURCE DIRECTIVE COORDINATION: BASIC THEORY

This chapter develops the theory upon which the Price-Resource Directive Coordination Procedure is based. The name of this procedure stems from the fact that the optimal dual variables or shadow prices and the right-hand side of the constraints vector, usually called resources vector, of the subsystems are both used to iteratively improve the allocation of the central resources to the subsystems. The procedure is not called a decomposition procedure because, after the actual reformulation of the total system into a two-level system, unlike the other methods described in $(7,62,66)$, there is no Master problem or Central system to be solved. Instead, a simple coordination mechanism is used to improve the partition of the central resources vector in such a manner, that after a finite number of "coordination meetings," the optimum resource allocation to the subsystems is determined.

The reformation of the linear program characterizing the total system is done following procedures developed for stochastic programming (61), and later rationalized for decomposition under the name of projection by Geoffrion (62). This type of problem manipulation, as was indicated in Chapter II, was also used by Zschau (58), and Silverman (50) under the name of primal decomposition. The details of 
this reformulation will be explained in the next section.

Some of the results presented in this chapter are not new but the proofs are. In particular, some of the results on the properties of the objective function of the Central Problem were proposed by Wets (49), but new detailed proofs consistent with the later developments are worked out here.

A very important contribution is the development of the important connection between the dual variables of a linear program and the directional derivatives of the objective function of this program, without utilizing the concept of subgradients as developed by Rockafellar (59), and Bazaraa (53). This contribution plus some of the results on the properties of the objective function of the Central System problem led to the development of a set of optimality conditions for nondifferentiable functions.

These optimality conditions constitute the basis of the PriceResource Directive Coordination procedure which will be developed in detail in the next chapter.

\section{THE BLOCK-ANGULAR STRUCTURED LINEAR SYSTEM}

The canonical form of the linear programming problem is

$$
\text { P.1 }\left[\begin{array}{r}
\text { maximize } z=\bar{c}^{\prime} \bar{x} \\
\text { subject to } A \bar{x} \leqslant \bar{b} \\
\bar{x}=\bar{o}
\end{array}\right.
$$

where $\vec{c}$ and $\bar{x}$ are $n-e l e m e n t$ vectors, $\bar{b}$ is an m-element vector and $A$ is an $\mathrm{m} \times \mathrm{n}$ matrix. This matrix tends to be structured in a block-angular 
form for large-scale systems (34), and if it does not, it can be transformed to the block-angular form according to procedures developeci by Weil and Kettler (35). Therefore the relations (3.1), (3.2) and (3.3) can be written as:

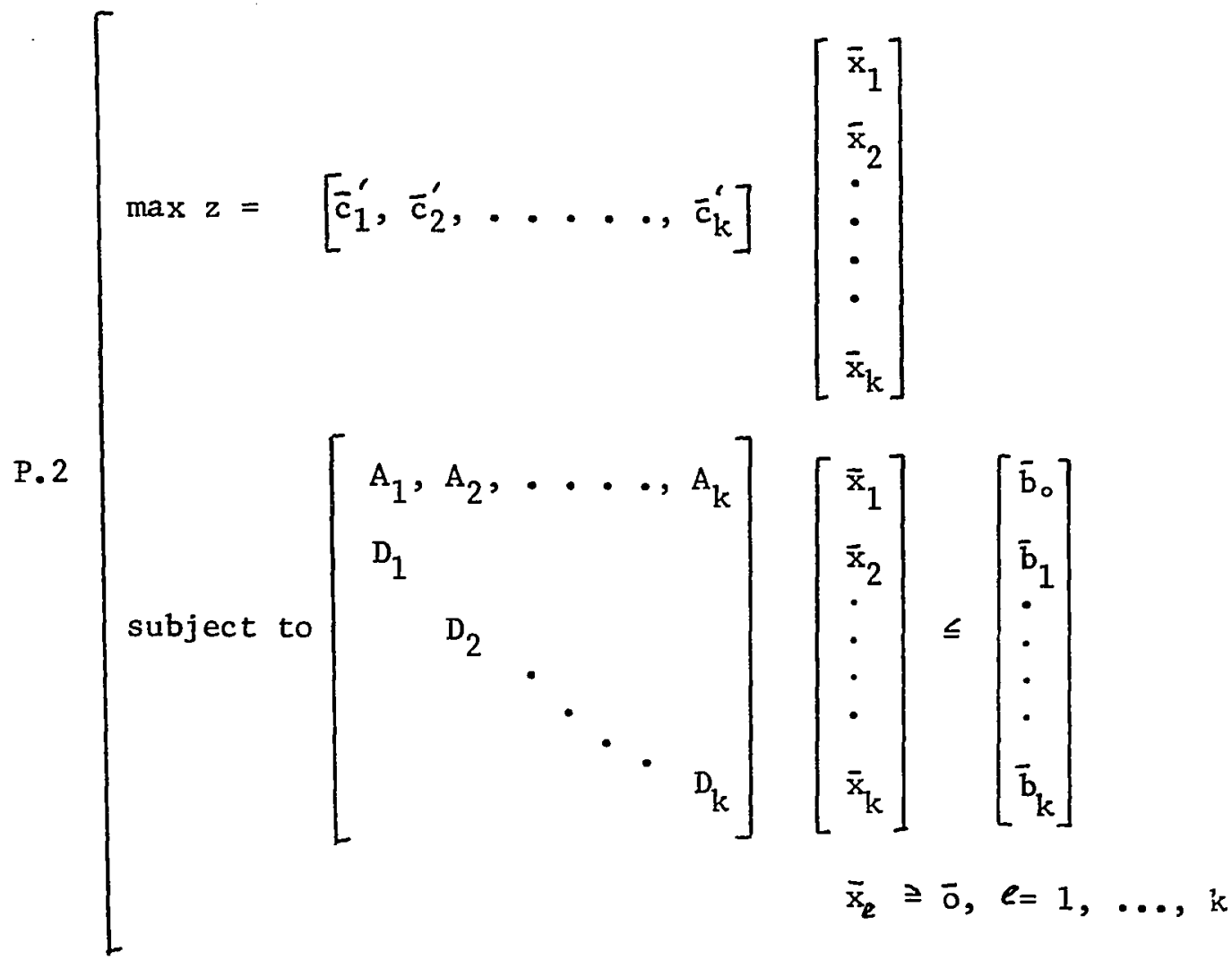

where:

$$
\begin{aligned}
& A_{e}=m_{0} x n_{e} \text { matrix } \\
& \mathrm{D}_{e}=\mathrm{m}_{e} \mathrm{xn}_{e} \text { matrix } \\
& \bar{c}_{l}^{\prime}=n_{l}-\text { element row vector } \quad \ell=1, \ldots, k \\
& \bar{x}_{c}=n_{e}-\text { element column vector } \\
& \bar{b}_{e}=m_{e}-\text { element column vector } \\
& \vec{b}_{0}=m_{0}-\text { element column vector }
\end{aligned}
$$


Therefore, for the problem P.2 the dimensions are:

$$
\begin{aligned}
& \text { number of constraints }=\mathrm{m}_{0}+\sum_{l=1}^{\ell=k} \mathrm{~m}_{l} \\
& \text { number of variables }=\sum_{\ell=1}^{e=k} \mathrm{n}_{e}
\end{aligned}
$$

The problem P.2 can be also written as:

$$
\begin{aligned}
& {\left[\operatorname{maximize} z=\sum_{l=1}^{l=k} \vec{c}_{e}^{\prime} \bar{x}_{e}\right.} \\
& \mathrm{D}_{e} \overline{\mathrm{x}}_{e} \leqslant \overline{\mathrm{b}}_{e}, \ell=1, \ldots, \mathrm{k} \\
& \bar{x}_{e} \geqslant \overline{0}, \ell=1, \ldots, k
\end{aligned}
$$

The objective function (3.10) and the system constraints (3.11) and (3.12) are linearly separable in the variables. This property of problem P. 3 makes possible the attempt to achieve a global (overa1I) optimum for problem P.3 in a multilevel fashion by coordinating the individual optimization of the sybsystems which result from the separability of problem P.3 after the partitioning of the vector $\bar{b}_{0}$. The development of the subsystems starts with this partitioning. 
he vector $\bar{b}_{0}$ is partitioned into $k$ arbitrary partitions such that

$$
\sum_{l=1}^{l=x} \bar{v}_{l} \leq \bar{b}_{0}
$$

where $\vec{v}_{e}$ is an $m_{0}$-element column vector.

Then the problem P.3 can be written as:

$P .4\left[\begin{array}{l}\operatorname{maximize} z_{e}=\bar{c}_{e}^{\prime} \bar{x}_{e} \\ \text { subject to } A_{e} \bar{x}_{e} \leq \bar{v}_{e} \quad \text { for } \ell=1, \ldots, k \\ D_{e} \bar{x}_{e} \leq \bar{b}_{e} \\ \bar{x}_{e} \geq \bar{o}\end{array}\right.$

The problem P.4 is completely separable and it can be considered as the decomposed equivalent of P. 3 since for each $\ell$ an independent linear programming problem can be solved giving rise to $k$ optimization subproblems.

The main consideration here is to find the optimum partition of the vector $\vec{b}_{0}$ such that the optimal solutions of the $k$ subproblems of P.4 sum up to the optimal solution of the problem P.3.

The Price-Resource Directive Coordination, to be developed, involves the iterative determination of the $m_{0}$-element vectors $\bar{v}_{1}, \ldots, \bar{v}_{k}$ such that the optimum partition can be achieved and henceforth the optimum for the overall system can be arrived at through the solutions of the subproblems. 
The optimum of problem P.4 depends on the partition of the vector $\bar{b}_{0}$. Each subproblem $\ell$, for $\ell=1, \ldots, k$ receives a vector $\bar{v}_{\ell}$ and according to that it optimizes itself. Therefore, the optimum of each subproblem is a function of the assigned vector $\vec{v}_{e}$, denoted by $\phi_{e}\left(\bar{v}_{e}\right)$, and thus the optimum partition of the vector $\bar{b}_{0}$ will be achieved when the sum of these functions is at a maximum subject to the feasibility constraint (3.14). This permits a reformation of $\mathrm{P} .4$ which is as follows:

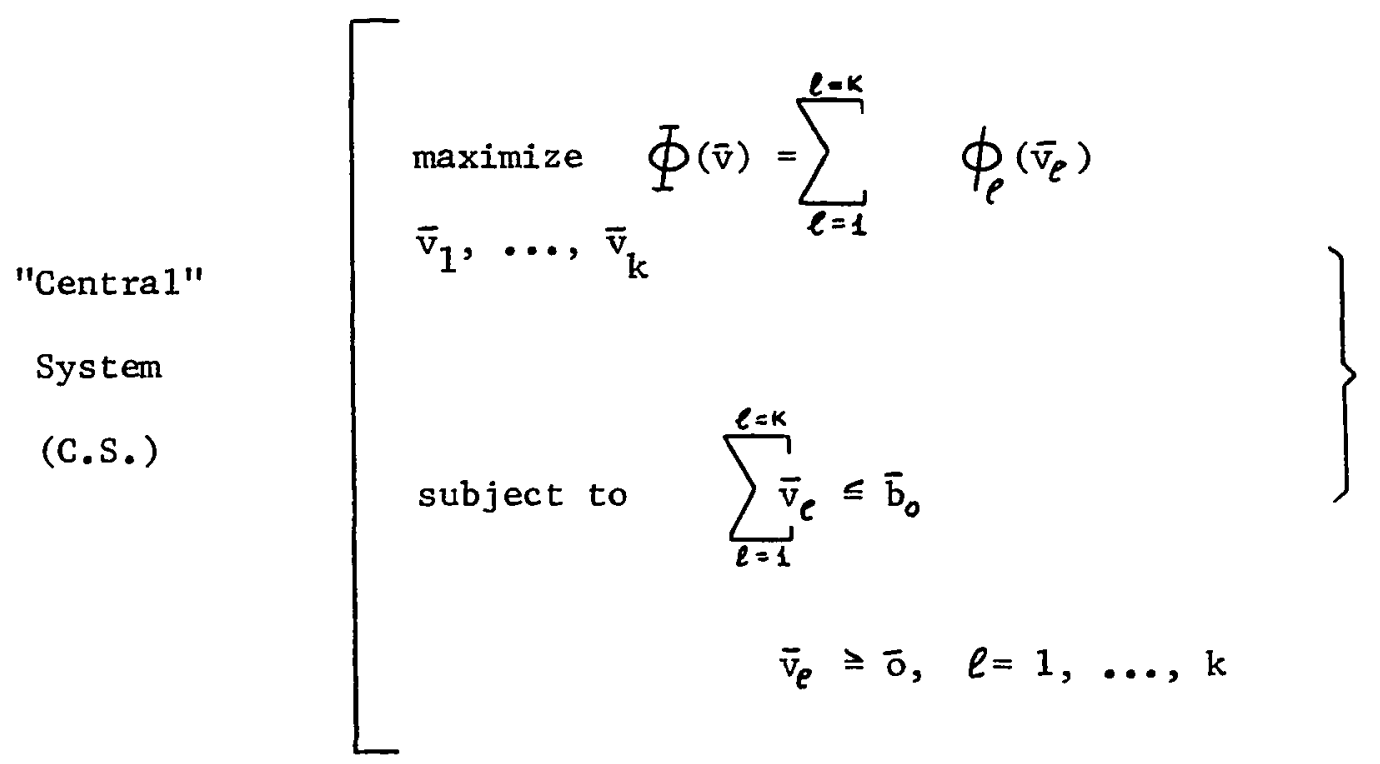

where in primal form

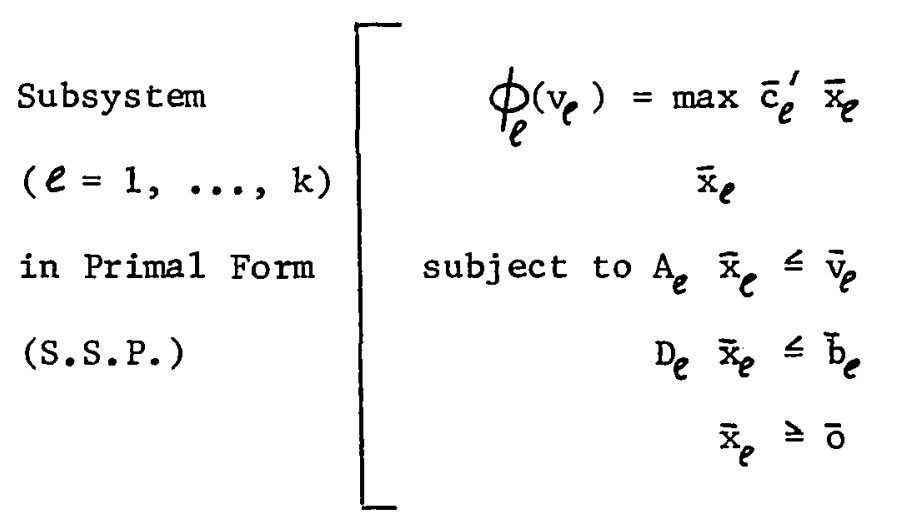


or in dual form

Subsysiem

$(e=1, \ldots, k)$

in Dual Form

(S.S.D.)

$$
\phi_{e}\left(\bar{v}_{e}\right)=\min _{\bar{w}_{e 1}, \bar{v}_{e}^{\prime} \bar{w}_{e l}}+\bar{b}_{e} \bar{w}_{e 2}
$$

subject to

$$
\begin{aligned}
{\left[A_{e}^{\prime}, D_{e}^{\prime}\right] } & {\left[\begin{array}{c}
\bar{v}_{e 1} \\
\bar{w}_{e 2}
\end{array}\right] \geqslant \bar{c}_{e} } \\
& \bar{w}_{e 1}, \bar{w}_{e 2} \geqslant \overline{0}
\end{aligned}
$$

This recasting of the initial problem follows a procedure which Geoffrior ( 36 , p. 30) calls problem manipulation and defines it as "the restatement of a given problem in an alternative form that is essentially equivalent but more amenable to solution."

Among the many benefits of this type of system decomposition into a Central system (C.S.) and Subsystems (S.S.) is the obvious one of the substantial reduction of the dimensionality of the problems requiring solution. The dimensions of the C.S. problem are:

$$
\begin{aligned}
& \text { number of constraints }=\mathrm{m}_{0} \\
& \text { number of variables }=\sum_{\ell=1}^{\ell=k} \mathrm{~m}_{0}=\mathrm{km}_{0}
\end{aligned}
$$

The dimensions of the S.S. problem $\ell$ are:

$$
\begin{aligned}
& \text { number of constraints }=\mathrm{m}_{0}+\mathrm{m}_{\ell} \\
& \text { number of variables }=\mathrm{n}_{\ell}
\end{aligned}
$$


Comparing (3.21) and (3.21a) with (3.8) we can see that depending on the number of subsystems there is a great reduction in the number of constraints which results in less computer storage requirements and greater speed of problem solution execution.

The equivalence of the C.S. problem and problem P.2 is formalized by the following theorem.

Theorem 3.1. The C.S. problem and the P.2 problem are equivalent in the sense that (i) the optimal objective values for C.S. and P.2 are equal, (ii) P.2 is unbounded if and only if the C.S. is unbounded, and (iii) if the C.S. has an optimal solution $\bar{v}^{0}=\left(\bar{v}_{j}^{0}, \ldots, \bar{v}_{k}^{0}\right)$ with $\bar{x}_{\rho}^{0}$ for $\ell=1, \ldots, k$ optimal in the S.S.'s, then $\bar{x}^{\circ}=\left(\bar{x}_{1}^{0}, \ldots, \bar{x}_{k}^{0}\right)$ is optimal in P.2.

Proof: The proof by contradiction is immediate.

The constraint sets (3.18) and (3.20) are the feasibility sets for the primal and dual variables $\overline{\mathrm{x}}_{e}, \overline{\mathrm{w}}_{\ell}$. Formally these can be given as follows:

$$
\begin{aligned}
& \mathrm{x}_{e}=\left[\begin{array}{l|l}
\overline{\mathrm{x}}_{e} \mid & \mathrm{A}_{e} \overline{\mathrm{x}}_{e} \leq \overline{\mathrm{v}}_{e}, \mathrm{D}_{e} \overline{\mathrm{x}}_{e} \leq \overline{\mathrm{b}}_{e}, \overline{\mathrm{x}}_{e} \geq \overline{\mathrm{o}}
\end{array}\right] \\
& \mathrm{w}_{e}=\left[\begin{array}{l|l}
\overline{\mathrm{w}}_{e} \mid & \left.\left(\mathrm{A}_{e}^{\prime}, \mathrm{D}_{e}^{\prime}\right) \overline{\mathrm{w}}_{e} \geq \overline{\mathrm{c}}_{e}, \overline{\mathrm{w}}_{e} \geq \overline{\mathrm{o}}, \overline{\mathrm{w}}_{e}=\left(\overline{\mathrm{w}}_{e 1}, \overline{\mathrm{w}}_{e 2}\right)\right]
\end{array}\right.
\end{aligned}
$$

-r.tained within the set $W_{l}$ is the set of the optimal dual variables $\overrightarrow{\mathrm{w}}_{\rho}^{0}$, which result by allocating a vector $\overrightarrow{\mathrm{v}}_{\rho}$ to the dual subsystem. This set is denoted by

$$
\mathrm{w}_{e}\left(\bar{v}_{e}\right) \longrightarrow \mathrm{w}_{e}
$$

Since the optimal dual variables define the function $\phi_{e}\left(\bar{v}_{e}\right)$, for each given $\bar{v}_{\ell}$, as it can be seen from (3.17) we have 


$$
\begin{aligned}
& \mathrm{w}_{e}\left(\overline{\mathrm{v}}_{e}\right)=\left[\overline{\mathrm{w}}_{e}^{0}\left(\overline{\mathrm{v}}_{e}\right) \mid \overline{\mathrm{w}}_{e}\left(\overline{\mathrm{v}}_{e}\right) \in \mathrm{w}_{e},\right. \\
& \left.\phi_{l}\left(\bar{v}_{e}\right)=\bar{w}_{e 1}^{0}\left(\bar{v}_{e}\right) \bar{v}_{e}+\bar{w}_{e 2}^{0}\left(\bar{v}_{l}\right) \bar{b}_{l} \text {, given } \bar{v}_{l} \in v_{l}\right]
\end{aligned}
$$

where

$$
\begin{aligned}
& \bar{w}_{e}^{0}\left(\bar{v}_{e}\right)=\left(\bar{w}_{e 1}^{0}\left(\bar{v}_{l}\right), \bar{w}_{e 2}^{0}\left(\bar{v}_{l}\right)\right) \\
& v_{e}=\left[\bar{v}_{e} \mid \bar{v}_{e} \leq \bar{b}_{0}, \bar{v}_{e} \geq \bar{o},\right. \\
& \text { solution exists for } \left.\ell^{t h}=\text { subsystem given } \vec{v}_{\ell}\right]
\end{aligned}
$$

The set $x_{e}$ and $w_{e}$ are expressed as the intersection of finitely many closed half spaces of the spaces $R^{n_{l}}$ and $R^{m_{0}+m_{l}}$. Therefore by definition they are polyhedral convex sets. The set $\mathrm{w}_{l}\left(\bar{v}_{e}\right)$ for each given vector $\bar{v}_{e}$ is also convex since it is the set of the optimal solution of some finite system of inequalities and equalities expressing the set $\mathrm{W}_{e}$.

The set $V_{e}$ is convex according to the following theorem.

Theorem 3.2. The set $\mathrm{V}_{C}$, as given by $(3.26)$, for which the S.S. problem has a solution is a convex set.

Proof: To prove that $\mathrm{V}_{e}$ is convex it must be shown that for given $\bar{v}_{e 1}, \bar{v}_{e 2} \in v_{l}$ and $0 \leq p \leq 1$ the relation

$$
\bar{v}_{e p}=(1-p) \bar{v}_{e 1}+p \bar{v}_{e z} \in v_{l}
$$

is also true, which means that at least one feasible solution should exist for $\bar{v}_{e_{p}}$. Since by assumption on the set $v_{e}$ a solution exists for the $\ell \stackrel{\text { sh }}{=} \mathrm{S} . \mathrm{S}$. problem, then the following relations taken from (3.18) are valid. 


$$
\begin{aligned}
& A_{e} \bar{x}_{e 1} \leqslant \bar{v}_{e 1}, D_{e} \bar{x}_{e 1} \leq \bar{b}_{e}, \bar{x}_{e 1} \geq \overline{0} \\
& A_{e} \bar{x}_{e 2} \leq \bar{v}_{e 2}, D_{e} \bar{x}_{e 2} \leqslant \bar{b}_{e}, \bar{x}_{e 2} \geq \overline{0}
\end{aligned}
$$

Now, if (3.28) is multiplied by $(1-p)$ and $(3.29)$ by $p$ and the results are added, we have

$$
\begin{aligned}
& A_{e}\left((1-p) \bar{x}_{e 1}+p \bar{x}_{e 2}\right) \leq(1-p) \bar{v}_{e 1}+p \bar{v}_{e 2} \\
& D_{e}\left((1-p) \bar{x}_{e 1}+p \bar{x}_{e 2}\right) \leq \bar{b}_{e} \\
& (1-p) \bar{x}_{e 1}+p \bar{x}_{e 2} \geq \overline{0}
\end{aligned}
$$

Since $(1-p) \bar{x}_{e 1}+p \bar{x}_{e 2} \geq \bar{o}$ we have a feasible solution for $(1-p) \bar{v}_{e 1}+p \bar{v}_{e 2}$ and therefore $(1-p) \bar{v}_{e 1}+p \bar{v}_{e 2} \in v_{e}$, which proves the theorem.

Definition 3.1. A set is closed if given a sequence $\bar{v}^{k} \in S$ such that

$$
\operatorname{limit}_{k \rightarrow \infty} \overline{\mathrm{v}}^{k}=\overline{\mathrm{v}}^{\infty}
$$

then

$$
\bar{v}^{\infty} \in \quad s
$$

Definition 3.2. The c1osure $\hat{S}$ of a set $S$ consists of a11 the limits of the converging sequences of $S$. The closure set $\hat{S}$ is a closed set. If a set $S$ is closed, then

$$
S=\hat{S}
$$


Examples: The set $s_{1}=\left[\begin{array}{l|l}\bar{x} & \bar{a}_{i}^{\prime} \bar{x} \leqslant b_{i}\end{array}\right] C R^{n}$ is closed, and $\mathrm{S}_{1}=\hat{\mathrm{S}}_{1}$.

The set $\mathrm{s}_{2}=\left[\mathrm{x} \mid \overline{\mathrm{a}}_{i}^{\prime} \overline{\mathrm{x}}<\mathrm{b}_{i}\right] \mathrm{C} \mathrm{R}^{\mathrm{n}}$ is not closed. Then its closure is the set $s_{1}$ and therefore $s_{1}=\hat{s}_{2}$ or $\mathrm{s}_{1}=\hat{\mathrm{s}}_{1}=\hat{\mathrm{s}}_{2}$.

According to the definitions above the sets $x_{\rho}, W_{l}, W_{l}\left(\vec{v}_{e}\right)$ for a given $\bar{v}_{e}$, and $v_{e}$ are all closed and $x_{e}=\hat{x}_{e}, w_{e}=\hat{W}_{e}, w_{e}\left(v_{e}\right)=$ $\hat{\mathrm{W}}_{e}\left(\mathrm{v}_{e}\right), \mathrm{v}_{e}=\hat{\mathrm{v}}_{e}$.

Also, the sets $x_{e}$ and $w_{e}$ are bounded from below since $\bar{x}_{e} \geq \bar{o}$, $\bar{w}_{e} \geqslant \bar{o}$ and the sets $W_{e}\left(v_{e}\right)$ and $v_{e}$ are bounded from above and below since $\bar{o} \leq \bar{v}_{e} \leq \bar{b}_{o}$.

Now, we can study the set of all the optimal dual variables for the whole range of the vector $\vec{v}_{e}$, which is $\overline{0} \leq \bar{v}_{e} \leq \bar{b}_{0}$. This set we denote by

$$
P=\bigcap_{i=1}^{i=r} w_{e}\left(\bar{v}_{e}^{i}\right), \bar{v}_{e}^{r}=\bar{b}_{0} \text { and } \bar{v}_{e}^{1}=\text { null vector }
$$

It is well known that the intersection of convex sets is a convex set (69) and henceforth $P$ is a convex set, and since each set $W_{e}\left(\bar{v}_{e}\right)$ is closed the set $P$ is closed. The following theorem will also prove that each set $W_{e}\left(\bar{v}_{e}\right)$ could be the closure set for a convergence sequence of the vector $\bar{v}_{e}$.

Theorem 3.3. By the closure of $\mathrm{P}$

$$
\begin{aligned}
& \lim \bar{w}_{e}^{0}\left(\bar{v}_{e}^{1}\right)=\bar{w}_{e}^{0}\left(\bar{v}_{e}^{t}\right) \\
& \bar{v}_{e}^{1} \longrightarrow \bar{v}_{l}^{t}
\end{aligned}
$$


then

$$
\overline{\mathrm{w}}_{e}^{0}\left(\bar{v}_{e}^{t}\right) \in \mathrm{w}_{e}\left(\overline{\mathrm{v}}_{e}^{t}\right)
$$

where

$1 \leq t \leq r$

Proof:

$$
\begin{aligned}
\phi\left(\bar{v}_{l}^{1}\right)= & \bar{w}_{l 1}^{0}\left(\bar{v}_{l}^{1}\right)^{\prime} \bar{v}_{l}^{1}+\bar{w}_{e 2}^{0}\left(\bar{v}_{l}^{1}\right)^{\prime} \bar{b}_{l} \\
= & \bar{w}_{e 1}^{0}\left(\bar{v}_{l}^{1}\right)^{\prime} \bar{v}_{l}^{1}+\bar{w}_{e 2}^{0}\left(\bar{v}_{l}^{1}\right)^{\prime} \bar{b}_{l}+\bar{w}_{e 1}^{0}\left(\bar{v}_{l}^{1}\right)^{\prime} \bar{v}_{l}^{t}- \\
& \bar{w}_{l 1}^{0}\left(\bar{v}_{l}^{1}\right)^{\prime} \bar{v}_{l}^{t} \\
= & \bar{w}_{e 1}^{0}\left(\bar{v}_{l}^{1}\right)^{\prime} \bar{v}_{l}^{t}+\bar{w}_{e 2}^{0}\left(\bar{v}_{l}^{1}\right)^{\prime} \bar{b}_{l}+\bar{w}_{e_{1}}^{0}\left(\bar{v}_{l}^{1}\right)^{\prime}\left(\bar{v}_{l}^{1}-\bar{v}_{l}^{t}\right)
\end{aligned}
$$

then

$$
\begin{aligned}
& \text { limit } \phi\left(\bar{v}_{e}^{1}\right)=\bar{w}_{e_{1}}^{0}\left(\bar{v}_{l}^{t}\right)^{\prime} \bar{v}_{l}^{t}+\bar{w}_{e 2}^{0}\left(\bar{v}_{l}^{t}\right)^{\prime} \bar{b}_{e} \\
& \vec{v}_{e}^{1} \longrightarrow \bar{v}_{e}^{t} \\
& =\phi\left(\bar{v}_{e}^{t}\right)
\end{aligned}
$$

which proves that the limit points of a set $w_{e}\left(\bar{v}_{e}\right)$ are elements of another set contained in $P$ and thus

$$
\overline{\mathrm{w}}^{0}\left(\bar{v}_{e}^{t}\right) \in \mathrm{w}_{e}\left(\overline{\mathrm{v}}_{e}^{t}\right)
$$

For the solution of the Central system problem it is paramount to study the behaviour and the properties of its objective function $\Phi$. This is the case because the explicit determination of this function is impossible in any real sense, since it entails the solution of each subsystem for each conceivable combination of the elements of 
the vector $\bar{v}_{e}$ ranging from $\bar{o}$ to $\vec{b}_{0}$. The properties of this function depend on the identification of the properties of the function $\phi_{l}\left(\vec{v}_{l}\right)$ for each subsystem. This problem will be dealt with in the next section.

\section{PROPERTIES OF THE FUNCTION $\Phi$}

The properties of the function $\phi_{e}\left(\bar{v}_{l}\right)$, upon which those of $\Phi$ depend, can be identified by a postoptimization analysis (37) of the solution of any one of the $k$ subsystems, as given in relations $(3.17)-(3.20)$ and subsequently by a study of the results of this analysis.

Assume that for a given arbitrary partition of $\vec{b}_{0}$, the vector $\bar{v}_{e}$ was allocated to the $\ell$ th subsystem which was then solved by the simplex method of linear programming. Thus the basic optimum vector $\overline{\mathrm{x}}_{\mathrm{Be}}^{0}$ was found to be

$$
\begin{aligned}
\bar{x}_{B C}^{0} & =B^{-1}\left[\begin{array}{c}
\bar{v}_{e} \\
\bar{b}_{l}
\end{array}\right] \\
& =\left[\begin{array}{c:c}
B_{e 1}^{-1} & B_{e 2}^{-1}
\end{array}\right]\left[\begin{array}{c}
\bar{v}_{e} \\
\bar{b}_{e}
\end{array}\right]
\end{aligned}
$$

where $\mathrm{B}^{-1}$ is the optimal basis inverse and $\mathrm{B}_{i}^{-1}, \mathrm{~B}_{2}^{-1}$ are its partition for $\bar{v}_{e}$ and $\bar{b}_{e}$ respectively. 
The optimal value of the objective function was found to be

$$
\begin{aligned}
\phi_{l}\left(\bar{v}_{e}\right) & =\bar{c}_{B e}^{\prime}\left[B_{1}^{-1}: B_{2}^{-1}\right]\left[\begin{array}{c}
\bar{v}_{l} \\
\bar{b}_{l}
\end{array}\right] \\
& =\bar{c}_{B e}^{\prime}\left[B_{1}^{-1} \bar{v}_{l}+B_{2}^{-1} \bar{b}_{l}\right] \\
& =\left(\bar{c}_{B e}^{\prime} B_{1}^{-1}\right)_{l}+\left(\bar{c}_{B e}^{\prime} B_{2}^{-1}\right) \bar{b}_{l} \\
& =\bar{w}_{l 1}^{0} \bar{v}_{l}+\bar{w}_{e 2}^{0} \bar{b}_{e}
\end{aligned}
$$

where $\bar{w}_{l 1}^{0}, \bar{w}_{e 2}^{0}$ are the vectors of the partitioned vector of the optimal dual variables (38), in other words $\bar{w}_{e 1}^{0}, \bar{w}_{e 2}^{0}=\bar{w}_{e}^{0}$. Now, if the vector $\bar{v}_{e}$ allocated to the eth subsystem is increased (decreased) by a vector $\bar{d}_{e}$ such that $\bar{v}_{e}+\bar{d}_{e} \leqslant \bar{b}_{0}$, the following basic optimum vector will result: 


$$
\begin{aligned}
& \left(\bar{x}_{B C}^{0}\right)_{\text {new }}=\left[\begin{array}{ll}
B_{e 1}^{-1} & B_{C 2}^{-1}
\end{array}\right]\left[\begin{array}{l}
\bar{v}_{C}+\bar{d}_{C} \\
\bar{b}_{e}
\end{array}\right] \\
& =B_{e l}^{-1}\left(\bar{v}_{e}+\bar{d}_{e}\right)+B_{e 2}^{-1} \bar{b}_{e} \\
& =\left(B_{e 1}^{-1} \bar{v}_{e}+B_{e 2}^{-1} \bar{b}_{e}\right)+B_{e 1}^{-1} \bar{d}_{e} \\
& =\bar{x}_{B e}^{0}+B_{e l}^{-1} \bar{d}_{e}
\end{aligned}
$$

Then the optimal value of the objective function becomes

$$
\begin{aligned}
& \oint_{l}\left(\bar{v}_{e}+\bar{d}_{e}\right)=\bar{c}_{B e}^{\prime}\left(\bar{x}_{B e}^{b}\right)_{\text {new }} \\
& =\bar{c}_{B C}^{\prime}\left[\overline{\mathbf{x}}_{B C}^{0}+B_{e 1}^{-1} \bar{d}_{e}\right] \\
& =\bar{c}_{b e}^{\prime}\left[B_{e l}^{-1} \bar{v}_{e}+B_{e l}^{-1} \bar{b}_{e}\right]+\bar{c}_{b e}^{\prime} B_{e l}^{-1} \bar{d}_{e} \\
& =\bar{w}_{e 1}^{0} \bar{v}_{e}+\bar{w}_{e 2}^{*} \bar{b}_{e}+\vec{w}_{e 1}^{*} \bar{d}_{e} \\
& =\phi(v)+\bar{w}_{e_{1}}^{0} \overline{\mathrm{d}}_{e}
\end{aligned}
$$

The optimality of the solution is not affected by the change in 
the vector $\bar{v}_{e}$ since the objective function coefficient vector $\bar{c}_{B e}$ is not altered, but the feasibility can be affected unless

$$
\left(\bar{x}_{B e}^{0}\right)_{\text {new }} \geqslant \overline{0}
$$

or by $(3.29)$

$$
\bar{x}_{\theta e}^{0}+B_{e 1}^{-1} \bar{d}_{e} \geq \overline{0}
$$

or

$$
-\mathrm{B}_{e 1}^{-1} \overline{\mathrm{d}}_{e} \leq \overline{\mathrm{x}}_{6 e}^{0}
$$

If any of the elements of the new basic optimum solution vector becomes negative, then a change in basis is required to ensure further feasibility. This necessity is carried out by some of the steps of the Dua1 Simplex Method (40).

We note from expression (3.38) that the function $\phi_{e}\left(\bar{v}_{\rho}\right)$ is linear over the convex set (3.39). We will prove that actually the function $\oint_{e}\left(\bar{v}_{e}\right)$ is finitely concave, piecewise - linear, and continuous over the set $V_{e}$ which by Theorem 3.2 was proved to be convex.

Theorem 3.4. The function $\oint_{e}\left(\bar{v}_{e}\right)$ is a finite concave continuous function on the convex set $\mathrm{V}_{e}$.

Proof: To prove the function $\phi_{e}\left(\bar{v}_{e}\right)$ concave we have to prove that

$$
\begin{gathered}
(1-p) \quad \phi_{e}\left(\bar{v}_{e 1}\right)+p \quad \phi_{e}\left(\bar{v}_{e 2}\right) \leq \\
\phi\left((1-p) \bar{v}_{e 1}+p \bar{v}_{e 2}\right)
\end{gathered}
$$


holds. Let $\overline{\mathrm{x}}_{\mathrm{el}}^{0}, \overline{\mathrm{x}}_{\mathrm{e} 2}^{0}$ be optimal solution vectors for the S.S. problem corresponding to $\bar{v}_{e 1}, \bar{v}_{e 2} \in v_{e}$. Then

$$
\begin{aligned}
& \phi_{e}\left(\bar{v}_{e 1}\right)=\bar{c}_{e}^{\prime} \bar{x}_{e 1}^{0} \\
& \phi_{e}\left(\bar{v}_{e 2}\right)=\bar{c}_{e}^{\prime} \bar{x}_{e 2}^{0}
\end{aligned}
$$

and by assumption

$$
(1-p) \bar{c}_{e}^{\prime} \bar{x}_{e 1}^{0}+p \bar{c}_{e}^{\prime} \bar{x}_{e 2} \leq \bar{c}^{\prime}\left((1-p) \bar{x}_{e_{1}}^{0}+p \vec{x}_{e 2}^{0}\right)
$$

and by (3.41), (3.42)

$$
\begin{aligned}
& (1-p) \quad \oint_{e}\left(\bar{v}_{e 1}\right)+p \\
& \vec{c}^{\prime}\left((1-p) \bar{x}_{e_{1}}^{0}+p \bar{x}_{e 2}^{0}\right)
\end{aligned} \quad \phi_{e}\left(\bar{v}_{e 2}\right) \leq
$$

By Theorem 3.2, $\bar{v}_{e p}=(1-p) \bar{v}_{e 1}+p \bar{v}_{e 2} \in v_{e}$ and $\overline{\mathrm{x}}_{e r}=(1-p) \overline{\mathrm{x}}_{e 1}^{0}+\mathrm{p} \overline{\mathrm{x}}_{e 2}^{0}$ is feasible but not necessarily optimal solution to the problem.

$$
\begin{aligned}
& \phi\left(\bar{v}_{e p}\right)=\left[\max \bar{c}_{e}^{\prime} \bar{x}_{e} \mid A_{e} \bar{x}_{e} \leq\right. \\
& \left.\bar{v}_{e p}, D_{e} \bar{x}_{e} \leqslant \bar{b}_{e}, \bar{x}_{e} \geq \bar{o}\right]
\end{aligned}
$$

whose optimal vector will be denoted by $\bar{x}_{\text {ep }}$. Therefore

$$
\max \bar{c}^{\prime} \bar{x}_{e r} \leq \max \bar{c}^{\prime} \bar{x}_{e p}=\phi_{e}\left(\bar{v}_{e p}\right)
$$

and by (3.43)

$$
(1-p) \quad \phi_{e}\left(\bar{v}_{e 1}\right)+p \quad \phi_{e}\left(\bar{v}_{e 2}\right) \leq \phi_{e}\left(\bar{v}_{e p}\right)
$$

or 


$$
\begin{gathered}
(1-p) \quad \phi_{e}\left(\bar{v}_{e 1}\right)+p \quad \phi_{e}\left(\bar{v}_{e 2}\right) \leq \\
\phi_{l}\left((1-p) \bar{v}_{e 1}+p \bar{v}_{e 2}\right)
\end{gathered}
$$

which proves concavity.

To prove that $\phi_{e}\left(\bar{v}_{e}\right)$ is finite it has to be proven that an upper bound on the $\oint_{e}\left(\bar{v}_{e}\right)$ exists for any given $\bar{v}_{e} \leq \bar{b}_{0}$. In other words it has to be proven that

$$
\phi_{e}\left(\bar{v}_{e}+\overline{\mathrm{d}}_{e}\right) \leq \phi_{l}\left(\overline{\mathrm{v}}_{e}\right)+\left(\overline{\mathrm{w}}_{e}^{0}\right) \overline{\mathrm{d}}_{e}
$$

is valid. Let

$$
\left(\bar{v}_{e}\right)=\max \bar{c}_{e}^{\prime} \bar{x}_{e}=\bar{c}_{e}^{\prime} \bar{x}_{e}^{0}=\left(\bar{w}_{e}^{0}\right) \bar{v}_{e}
$$

then if the vector $\vec{v}_{e}$ is increased by $\overrightarrow{\mathrm{d}}_{e}$ from postoptimality analysis (37) we know that the dual solution

$$
\bar{w}_{e}^{0}\left(\bar{v}_{e}+\bar{d}_{e}\right)
$$

is not necessarily optimal, therefore from duality theory (39, p. 479).

$$
\bar{c}_{e}^{\prime}\left(\bar{x}_{e}\right)_{n \in w} \leqslant \bar{w}_{e}^{0}\left(\bar{v}_{e}+\bar{d}_{e}\right)
$$

Now, subtracting (3.47) and the above we have

$$
\bar{c}_{e}\left(\bar{x}_{e}\right)_{\text {new }}-\bar{c}_{e}^{\prime} \bar{x}_{e} \leq \bar{w}_{e}^{0}\left(\bar{v}_{e}+\overline{\mathrm{d}}_{e}\right)-\left(\overline{\mathrm{w}}_{e}^{0}\right)^{\prime} \overline{\mathrm{v}}_{e}
$$

or

$$
\phi_{e}\left(\bar{v}_{e}+\overrightarrow{\mathrm{d}}_{e}\right)-\phi_{e}\left(\overline{\mathrm{v}}_{e}\right) \leq\left(\overline{\mathrm{w}}_{e}^{0}\right)^{\prime} \overline{\mathrm{d}}_{e}
$$


37

or

$$
\phi_{e}\left(\overline{\mathrm{v}}_{e}+\overline{\mathrm{d}}_{e}\right) \leq \phi_{e}\left(\overline{\mathrm{v}}_{e}\right)+\left(\overline{\mathrm{w}}_{e}^{0}\right)^{\prime} \overline{\mathrm{d}}_{e}
$$

Continuity is proven by the fact that the function $\phi_{e}\left(\bar{v}_{e}\right)$ is concave over the set $V_{\rho}$, which is convex, which implies that

$$
\phi_{e}\left(\bar{v}_{e}\right) \text { is continuous at all interior points of that set. Then }
$$

there exists a sequence of interior points $\overline{\mathrm{v}}_{e}^{k}$ such that

$\lim \overline{\mathrm{v}}_{e}^{k}=\overline{\mathrm{v}}_{e}^{\cdot}$.

$k \rightarrow \infty$

Now, by concavity we have nonincreasing slopes and therefore since

$$
\bar{v}_{e}^{k} \leqslant \bar{v}_{e}^{*}
$$

or

$$
\left(\bar{v}_{e}^{k}-\bar{v}_{e}^{\prime}\right) \leq \bar{o}
$$

we have

$$
\overline{0} \leq \bar{w}_{e}^{0}\left(\bar{v}_{e}^{*}\right) \leq \bar{w}_{e}^{0}\left(\bar{v}_{e}^{x}\right)
$$

which if it is multiplied by $\left(\bar{v}_{e}^{k}-\bar{v}_{e}^{*}\right)$, which is negative, will result in

$$
0 \geq \bar{w}_{e}^{0}\left(\bar{v}_{e}^{*}\right)^{\prime}\left(\bar{v}_{e}^{k}-\bar{v}_{e}^{0}\right) \geq \bar{w}_{e}^{0}\left(\bar{v}_{e}^{k}\right)^{\prime}\left(\bar{v}_{e}^{k}-\bar{v}_{e}^{0}\right)
$$

and since $\phi_{e}\left(\bar{v}_{e}^{*}\right)-\phi_{e}\left(\bar{v}_{e}^{k}\right) \geq 0$, we have

$$
\phi_{e}\left(\bar{v}_{e}^{*}\right)-\phi_{e}\left(\bar{v}_{e}^{k}\right) \geq \bar{w}_{e}^{0}\left(\bar{v}_{e}^{k}\right)^{\prime}\left(\bar{v}_{e}^{k}-\bar{v}_{e}^{*}\right)
$$

or 


$$
\phi_{l}\left(\bar{v}_{l}^{k}\right)-\phi_{l}\left(\bar{v}_{e}^{*}\right) \leq \bar{w}_{l}^{0}\left(\bar{v}_{l}^{k}\right)^{\prime}\left(\bar{v}_{e}^{*}-\bar{v}_{l}^{k}\right)
$$

Then if we multiply (3.50) by $\bar{v}_{e}^{k}$, we have

$$
\bar{w}_{e}^{0}\left(\bar{v}_{e}^{0}\right)^{\prime} \bar{v}_{e}^{k} \leqslant \bar{w}_{e}^{0}\left(\bar{v}_{e}^{k}\right)^{\prime} \bar{v}_{l}^{k}
$$

Now, if we add to both sides of $(3.52)$ the value $-\overline{\mathrm{w}}_{e}^{0}\left(\overline{\mathrm{v}}_{e}^{\bullet}\right)^{\prime} \overrightarrow{\mathrm{v}}_{l}^{0}$ the result is

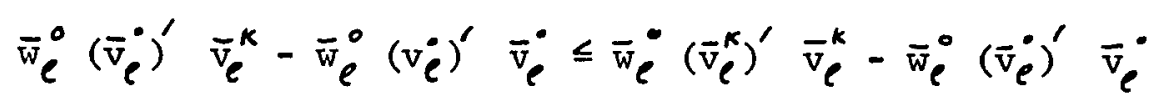

or

$$
\overline{\mathrm{w}}_{e}^{0}\left(\overline{\mathrm{v}}_{e}\right)^{\prime}\left(\overline{\mathrm{v}}_{e}^{\mathrm{k}}-\overline{\mathrm{v}}_{\mathrm{e}}^{*}\right) \leq \phi_{e}\left(\overline{\mathrm{v}}_{e}^{k}\right)-\phi_{\rho}\left(\overline{\mathrm{v}}_{\rho}^{*}\right)
$$

Combining the above result with (3.51) we have

$$
\begin{aligned}
& \bar{w}_{e}^{0}\left(\bar{v}_{e}^{*}\right)^{\prime}\left(\bar{v}_{e}^{k}-\bar{v}_{e}^{*}\right) \leq \phi_{e}\left(\bar{v}_{l}^{k}\right)-\phi_{l}\left(\bar{v}_{e}^{*}\right) \leq \\
& \bar{w}_{e}^{0}\left(\bar{v}_{e}^{k}\right)^{\prime}\left(\bar{v}_{e}^{*}-\bar{v}_{e}^{k}\right)
\end{aligned}
$$

The above result proves the continuity of the function since if

$$
\lim _{k \rightarrow \infty} \overline{\mathrm{v}}_{e}^{k}=\overline{\mathrm{v}}_{e}
$$

the relation (3.53) implies

$$
\lim _{k \rightarrow \infty} \phi_{e}\left(\bar{v}_{e}^{k}\right)=\phi_{e}\left(\bar{v}_{e}^{*}\right)
$$

which is the definition of function continuity. 
39

Theorem 3.5. The function $\phi_{e}\left(\bar{v}_{e}\right)$ is piecewise linear.

Proof: Assume an initial allocation of a vector $\bar{v}_{e_{0}}$ to the S.S. problem with $\phi_{e}\left(\bar{v}_{e o}\right)$ as optimum and then assume that an increase $\overline{\mathrm{d}}_{e}$ is given to $\overline{\mathrm{v}}_{e_{0}}$, such that

$$
\overline{\mathrm{v}}_{e 1}=\left(\overline{\mathrm{v}}_{e_{0}}+\overline{\mathrm{d}}_{e}\right) \leq \overline{\mathrm{b}}_{0}
$$

Then the relations $(3.36)-(3.39)$ can be generalized to the following expressions :

$$
\phi_{l}\left(\bar{v}_{e_{1}}\right)=\phi_{l}\left(\bar{v}_{e 0}\right)+\left(\bar{w}_{e 1}^{0}\right)_{0}^{\prime}\left(\bar{v}_{e_{1}}-\bar{v}_{e_{0}}\right)
$$

is valid over the set

$$
\left(E_{e 2}^{-1}\right) \bar{b}_{e}+\left(B_{e 1}^{-1}\right)_{0} \bar{v}_{e 0}+\left(B_{e l}^{-1}\right)_{0}\left(\bar{v}_{e 1}-\bar{v}_{e 0}\right) \geq \overline{0}
$$

Now, if $\vec{v}_{e 1}$ increases up to $\vec{b}_{0}$ and the linear program is still feasible, in other words (3.55) remains valid, then the function $\phi_{e}\left(\bar{v}_{e}\right)$, as it can be seen from (3.54), it is a linear function, with $\left(\bar{w}_{l 1}^{0}\right)_{0}$ constant, over the set $V_{l}$. But if at any time (3.55) becomes invalid then the S.S. problem needs reoptimization which generates a new basis inverse and new dual variables since

$$
\bar{w}_{e 1}^{0}=\bar{c}_{B C}^{\prime} B_{e 1}^{-1}
$$

Therefore, given a vector $\vec{v}_{e_{2}}$ which forces infeasibility and consequently reoptimization the relation (3.54) becomes

$$
\begin{aligned}
& \phi_{e}\left(\bar{v}_{e 2}\right)=\phi_{e}\left(\bar{v}_{e 0}\right)+\left(\bar{w}_{e 1}^{0}\right)_{0}^{\prime}\left(\bar{v}_{e 1}-\bar{v}_{e 0}\right)+ \\
& \left(\bar{w}_{e 1}^{0}\right)_{1}^{\prime}\left(\bar{v}_{e 2}-\bar{v}_{e 1}\right)
\end{aligned}
$$


and it is valid over the new convex set

$$
(B-1)_{1} \bar{b}_{e}+\left(B_{l 1}^{-1}\right)_{1} \bar{v}_{l 1}+\left(B_{e 1}^{-1}\right)_{1}\left(\bar{v}_{e 2}-\bar{v}_{e 2}\right) \geq \overline{0}
$$

If there are $r-1$ such points of infeasibility and reoptimization, the relation $(3.54)$ becomes

$$
\phi_{e}\left(\bar{v}_{e r}\right)=\phi_{e}\left(\bar{v}_{e 0}\right)+\sum_{q=0}^{r-1}\left(\bar{w}_{e 1}^{0}\right)_{q}^{\prime}\left(\bar{v}_{e}, q+1 \quad-v_{e, q}\right)
$$

which by definition is a piecewise-linear function.

The above theorems established that the function $\phi_{e}\left(\vec{v}_{e}\right)$ for $\ell=1, \ldots, k$ is a finitéte 2 piecewise-1inear_continuous,

function.

The slopes of the linear pieces are the dual variables or the shadow prices and they are discontinuously decreasing as the value of $\vec{v}_{e}$ is increasing. Figures 1 and 2 give pictorial representations of the function $\phi_{e}\left(\bar{v}_{e}\right)$ and the dual variable $\bar{w}_{e}$ for a system with one resource.

Now, we are ready to prove that the function $\Phi(\vec{v})$ is finite, piecewise-linear continuous and concave over the set $\mathrm{V}$ which is convex being the intersection of the sets $v_{e}$ for $l=1, \ldots, k$. 


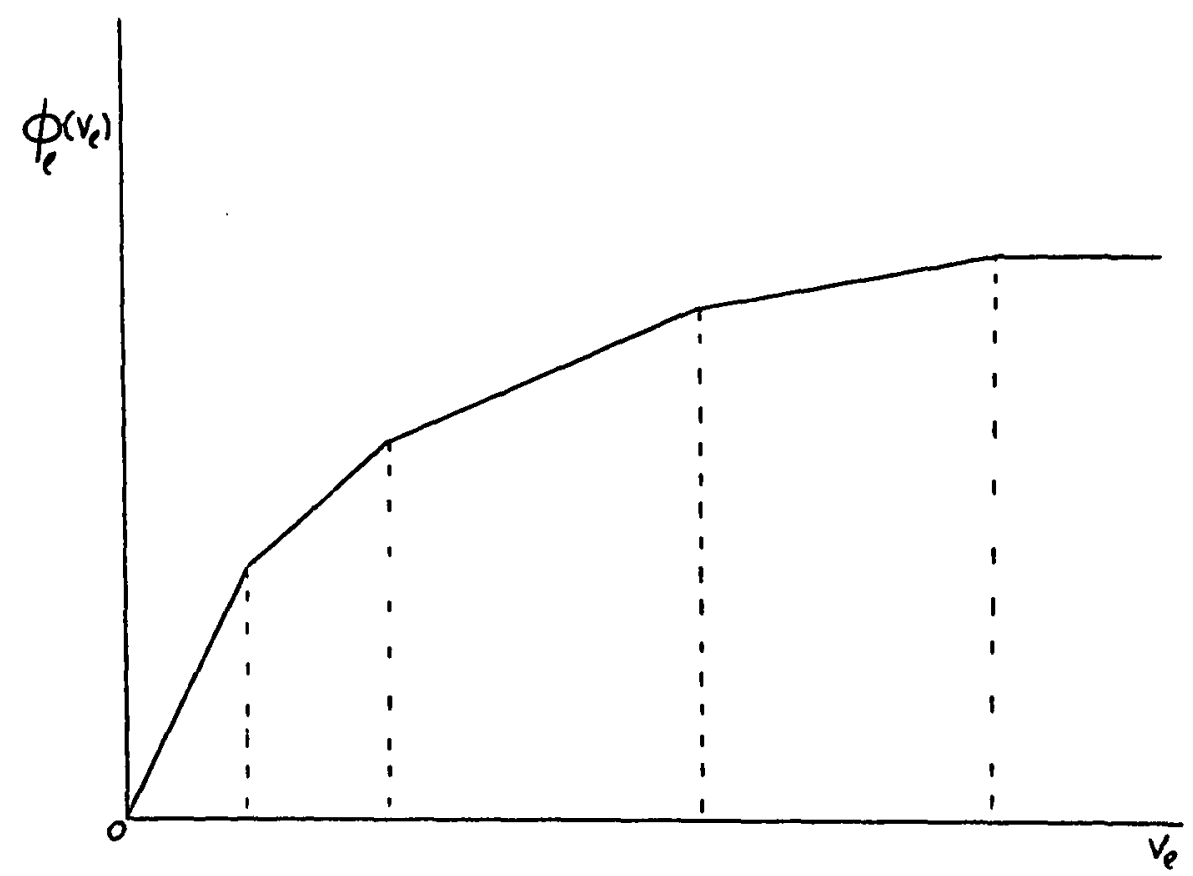

Figure 1. Subsystem objective function variation with change in the resources.

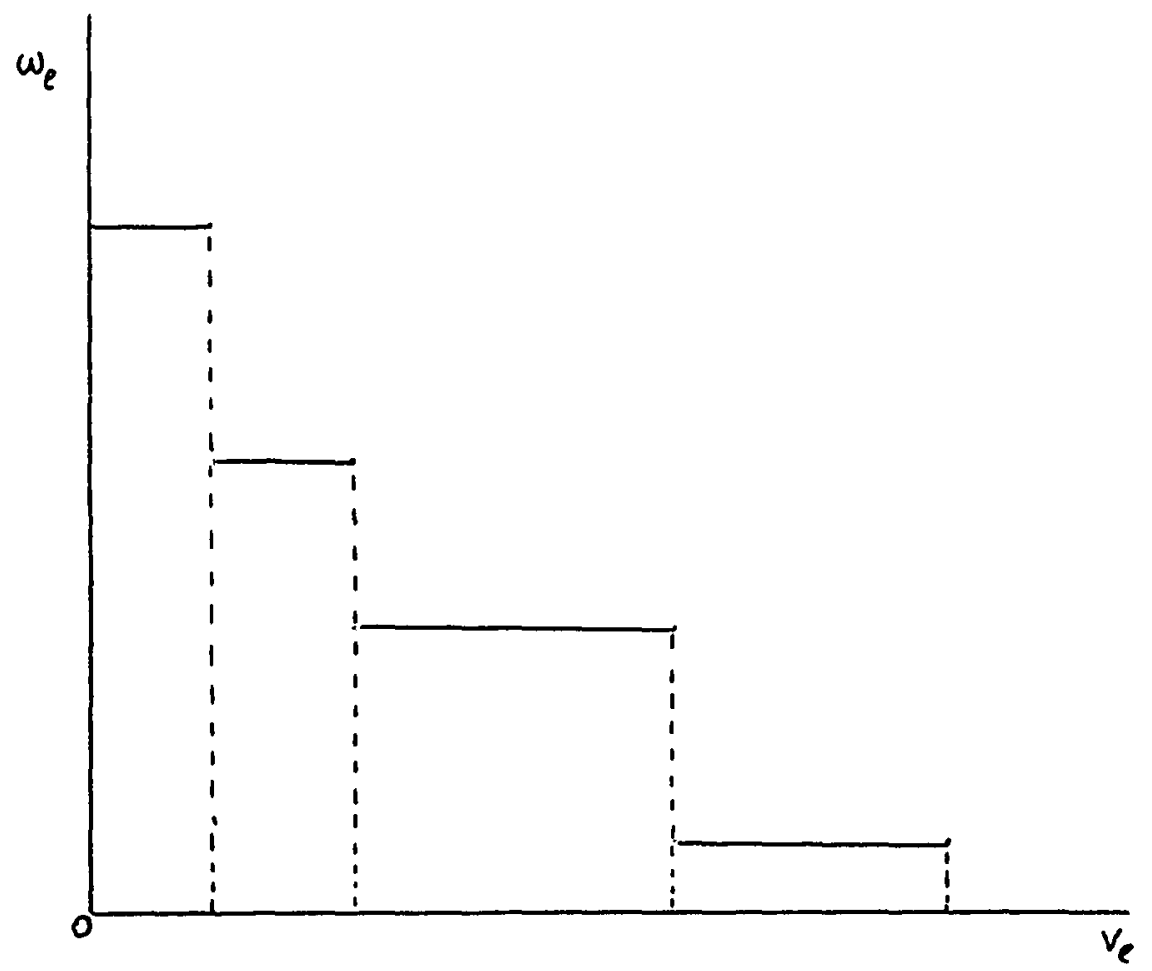

Figure 2. Subsystem shadow price variation with change in the resources. 
42

Theorem 3.6. The function $\Phi(\bar{v})=\sum_{l=1}^{\operatorname{lak}} \phi_{l}\left(\bar{v}_{l}\right)$ is piecewise-1inear.

Proof: From (3.59) of Theorem 3.5 we have

$$
\phi_{e}\left(\vec{v}_{e r}\right)=\phi_{l}\left(\bar{v}_{e 0}\right)+\sum_{q=0}^{r-1}\left(\bar{w}_{e l}^{0}\right)_{q}^{\prime}\left(\bar{v}_{e, q+1}-\bar{v}_{e, q}\right)
$$

and summing over $\ell=1, \ldots, k$

$$
\begin{aligned}
& \sum_{l=1}^{l=k} \phi_{l}\left(\bar{v}_{e r}\right)=\sum_{l=1}^{\ell=k} \phi_{l}\left(\bar{v}_{e o}\right)+\sum_{l=1}^{l=k} \sum_{q=0}^{r-1}\left(\bar{w}_{e 1}^{0}\right)_{q}^{\prime} \\
& \left(\bar{v}_{e, q+1}-\bar{v}_{e, q}\right)
\end{aligned}
$$

or

$$
\Phi\left(\bar{v}_{r}\right)=\Phi\left(\bar{v}_{0}\right)+\sum_{q=0}^{r-1} \bar{w}_{q}^{\prime}\left(\bar{v}_{q+1}-\bar{v}_{q}\right)
$$

where

$$
\bar{v}=\left[\begin{array}{c}
\bar{v}_{1} \\
\bar{v}_{2} \\
\cdot \\
\dot{\mathrm{v}}_{k}
\end{array}\right] \quad \overline{\mathrm{w}}^{\prime}=\text { sum of whole or part pieces of the dual }
$$


43

The relation (3.60) proves that the function $\Phi(\bar{v})$ is piecewise-1inear.

Theorem 3.7. The function $\Phi(\bar{v})$ is concave on the set V.

Proof: From the concavity of the function $\oint_{e}\left(\bar{v}_{l}\right)$ we have

$(1-p) \quad \phi_{l}\left(v_{e 1}\right)+p \quad \phi_{l}\left(\bar{v}_{e 2}\right) \leq \phi_{l}\left((1-p) \bar{v}_{e 1}+p \bar{v}_{e 2}\right)$

and for $\bar{v}_{e 3}=(1-p) \bar{v}_{e 1}+p \bar{v}_{e 2} \in v_{e}$

$(1-p) \quad \phi_{e}^{\left(\bar{v}_{e 1}\right)+p} \quad \phi_{e}^{\left(\bar{v}_{e 2}\right)} \leqslant \phi_{e}\left(\bar{v}_{e 3}\right)$

hence

$$
(1-p) \sum_{l=1}^{l=k} \phi_{l}\left(\bar{v}_{l 1}\right)+p \sum_{l=1}^{l=k} \phi_{l}\left(\bar{v}_{l l}\right) \leq \sum_{l=1}^{l=k} \phi_{l}\left(\bar{v}_{e 3}\right)
$$

and therefore

$$
(1-p) \quad \Phi\left(\bar{v}_{1}\right)+p \quad \Phi\left(\bar{v}_{2}\right) \leq \Phi\left(\bar{v}_{3}\right)
$$

where

$$
\bar{v}_{1}=\left[\begin{array}{c}
\bar{v}_{11} \\
\bar{v}_{21} \\
\cdot \\
\cdot \\
\bar{v}_{k 1}
\end{array}\right], \bar{v}_{2}=\left[\begin{array}{c}
\bar{v}_{12} \\
\bar{v}_{22} \\
\cdot \\
\cdot \\
\bar{v}_{k 2}
\end{array}\right] \text { and } \bar{v}_{3}=\left[\begin{array}{c}
(1-p) \bar{v}_{11}+p \bar{v}_{12} \\
(1-p) \bar{v}_{21}+p \bar{v}_{22} \\
\cdot \\
\cdot \\
(1-p) \bar{v}_{k 1}+p \bar{v}_{k 2}
\end{array}\right]
$$


Since the function $\Phi(\bar{v})$ is piecewise-linear, it becomes apparent that the gradient of $\Phi(\bar{v})$ does not exist at the points where the slopes change; in other words, the function $\Phi(\bar{v})$ is not differentiable at all points. This invalidates the so-called Gradient Method for the solution of the Central System problem. Fortunately, the directiona1 derivatives of $\Phi(\bar{v})$ and $\phi_{e}\left(\bar{v}_{e}\right)$ exist as is shown in the theorem following the definitions of a directional derivative and a direction vector.

Definition 3.3. Let the function $\Phi(\bar{v})$ be defined on the set $\mathrm{V}$ and assume $\overline{\mathrm{d}} \in \mathrm{R}^{\mathrm{km} m_{0}}$ such that $\|\overline{\mathrm{d}}\|=1$. The directiona 1 derivative of $\Phi(\bar{v})$ at $\bar{v}$ in the direction $\bar{d}$ is defined to be the 1 imit

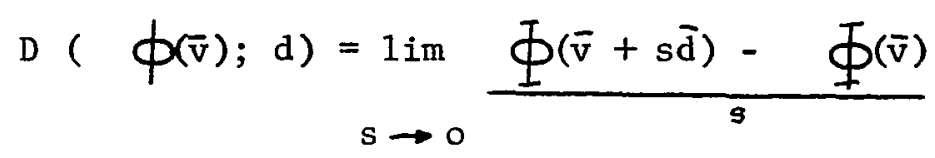

whenever it exists.

Definition 3.4. Assume $\overline{\mathrm{v}} \in \mathrm{R}^{\mathrm{km} m_{0}}, \overline{\mathrm{d}} \in \mathrm{R}^{\mathrm{km} m_{0}}, \mathrm{~S} \in \mathrm{R}$ and $0 \leq s \leq+\infty, \bar{d} \neq \overline{0}$, then a new vector can be found $\bar{v}^{*} \epsilon R^{k m_{0}}$ where

$$
\overline{\mathrm{v}}^{*}=\overline{\mathrm{v}}+\mathrm{s} \overline{\mathrm{d}}
$$

which describes a ray emanating from the point $\bar{v}$ in the direction $\bar{d}$. Any vector $\overrightarrow{\mathrm{d}} \in \mathrm{R}^{\mathrm{km}}$ can serve as a direçtion veçtor in this sense. A direction vector becomes a feasible_direction vector if for a given vector $\bar{v} \in R^{\mathrm{km}} \cap \mathrm{S}$ the new vector $\overline{\mathrm{v}}^{*}$ is also an element of the set $S$, i.e. 


$$
\bar{v}^{*}=(\bar{v}+s \bar{d}) \in R^{k m_{0}} \cap S
$$

The set of all feasible direction vectors at a given $\bar{v}$ is denoted by

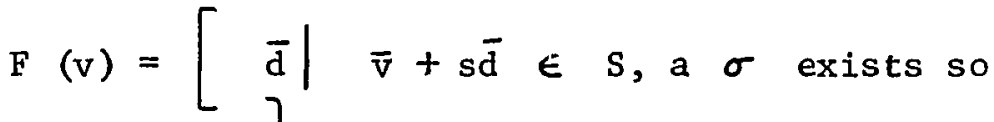

$$
\begin{aligned}
& \sigma \geq \mathrm{s} \geq 0]
\end{aligned}
$$

Verbally, $\overline{\mathrm{d}} \in \mathrm{F}(\overline{\mathrm{v}})$ if a small movement from $\overline{\mathrm{v}} \in \mathrm{S}$ in the direction $\overline{\mathrm{d}}$ produces $\overline{\mathrm{v}}^{*}=(\overline{\mathrm{v}}+\mathrm{s} \overline{\mathrm{d}}) \in \mathrm{S}$.

For the Central System problem, a feasible direction $\bar{d}$ for

$\overrightarrow{\mathrm{v}} \in \mathrm{V}$ will be called a usable fe feasible $\underline{\text { direction }}$ if

$$
D(\Phi(\bar{v}) ; d)>0
$$

holds. It can be easily seen from the definition of the directional derivative that a usable feasible direction increases the value of the objective function, i.e.

$$
\left.\Phi^{(\bar{v}}+s \bar{d}\right)>\Phi^{(\bar{v})}
$$

The following theorem proves the existence of a directional derivative for $\phi_{e}\left(\bar{v}_{e}\right)$ and $\Phi(\bar{v})$, since both of them are concave.

Theorem 3.8. The directional derivative of the finite concave function $\oint_{e}\left(\bar{v}_{e}\right)$ exists for each direction vector $\overline{\mathrm{d}}_{e}$, and it is positively homogeneous convex function of $\bar{d}_{e}$, with

$$
D_{e}\left(\phi_{e}\left(\bar{v}_{e}\right) ; \overline{0}\right)=0
$$

and 


$$
-\mathrm{D}_{e}\left(\phi_{e}\left(\overline{\mathrm{v}}_{e}\right) ;-\overline{\mathrm{d}}_{e}\right) \leq \mathrm{D}_{e}\left(\phi_{e}\left(\overline{\mathrm{v}}_{e}\right) ; \overline{\mathrm{d}}_{e}\right)
$$

The proof is given by Rockafellar (59).

Now, to summarize the results about the properties of the functions $\Phi(\bar{v})$ and $\phi_{e}\left(\bar{v}_{e}\right)$ it should be said that these functions are:

(i) concave

(ii) finite

(iii) piecewise-1inear

(iv) continuous

(v) nondifferentiable in genera1

(vi) directionally differentiable everywhere

The importance attached in finding these properties of the function $\Phi(\bar{v})$ lies in the fact that it is almost impossible to derive an explicit expression for this function. The task requires the solution of the subsystems an innumerable number of times which for all practical purposes is not attainable.

The properties listed above plus the properties of the sets of the primal and dual variables developed in this section will be used, in conjunction with some other theorems, in the development of the optimality conditions, on which the price-resource directive decomposition is based. 


\section{OPTIMALITY CONDITIONS FOR THE CENTRAL SYSTEM PROBLEM}

The problem of optimality conditions for the general nonlinear programming system with differentiable real-valued functions defined on the nonnegative orthant of Euclidean n-space was first approached and partially solved by F. John (42). The definitive treatment of this subject was done in 1951 by Kuhn and Tucker (43) and since then the optimality conditions for nonlinear programming are known as the KuhnTucker conditions. Other authors refined certain aspects of the derivation of the optimality conditions $(44,45)$, but the first real improvement in the theory occurred with the withdrawal of the differentiability assumption by Danskin (47) and Bram (48). They developed optimality conditions for the nonlinear programming problem utilizing directional derivatives. Their method was used by Zschau $(56,58)$ and Silverman $(50,51)$ to develop new resource-directive decomposition procedures. A more abstract formulation of the optimality conditions for the nonlinear programming problem assuming convexity only was given by Rockafellar (59) and an extension of this formulation replacing convexity with a weaker assumption of supportabilicy was developed by Bazaraa et. a1. (53).

In this section the necessary and sufficient conditions for an optimal solution to the Central System problem will be derived.

The Saddle Point Problem and Optimality

Consider the Central System problem written as 


$$
\text { maximize } \phi(\bar{v})=\sum_{\ell=1}^{l_{a} k} \phi_{l}\left(\bar{v}_{l}\right)
$$

subject to

$$
\bar{g}(\bar{v})=\left[\bar{b}_{0}-H \bar{v}\right] \geq \overline{0}
$$

where: $G=m_{0} \times \mathrm{km}_{0}$ matrix of the form

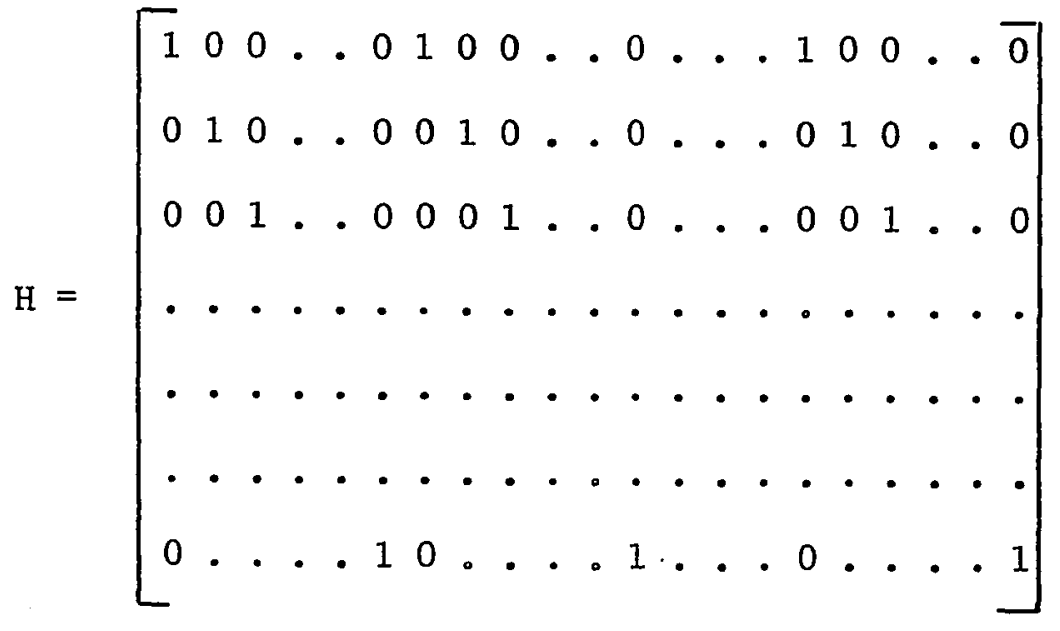

and $\quad \bar{v}=k m_{0}$ - element vector of the form

$$
\bar{v}=\left[\begin{array}{c}
\bar{v}_{1} \\
\bar{v}_{2} \\
\cdot \\
\cdot \\
\bar{v}_{k}
\end{array}\right]
$$

Associated with this problem is the real-valued function $\mathrm{L}\left(\overline{\mathrm{v}}_{1}, \ldots\right.$, $\left.\bar{v}_{k}, \bar{u}\right)$ of the $m$-vectors $\bar{v}_{l}$, for $l=1, \ldots, k$, and the $m_{0}$-vector $\bar{u}$. 
This function is called the Lagrangean Function and is defined by the following equation:

$$
L\left(\bar{v}_{1}, \ldots, \bar{v}_{k}, \bar{u}\right)=\sum_{l=1}^{l=k} \phi_{l}\left(\bar{v}_{l}\right)+\bar{u}^{\prime} \bar{g}(\bar{v})
$$

Definition 3.5. A sadd1e_point of the Lagrangean Function is defined to be a point $\left(\bar{v}_{1}^{0}, \ldots, \bar{v}_{k}^{0}, \bar{u}^{0}\right), \bar{v}_{e}^{0} \geq \bar{o}$, for $\ell=1, \ldots, k$, $\overline{\mathbf{u}}^{\circ} \geq \overline{0}$ such that

$$
\begin{aligned}
& L\left(\bar{v}_{1}, \ldots, \bar{v}_{k}, \bar{u}^{0}\right) \leq L\left(\bar{v}_{1}^{0}, \ldots, \bar{v}_{k}^{0}, \bar{u}^{0}\right) \leq \\
& L\left(\bar{v}_{1}^{0}, \ldots, \bar{v}_{k}^{0}, \bar{u}\right)
\end{aligned}
$$

or

$$
\begin{aligned}
& \Phi(\bar{v})+\left(\bar{u}^{0}\right)^{\prime} \bar{g}(\bar{v}) \leq \Phi\left(\bar{v}^{0}\right)+\left(\bar{u}^{0}\right)^{\prime} \bar{g}\left(\bar{v}^{0}\right) \leq \\
& \Phi\left(\bar{v}^{0}\right)+(\bar{u})^{\prime} \bar{g}\left(\bar{v}^{0}\right)
\end{aligned}
$$

The above expressions give the constrained saddle point of the Lagrangean Function. It will be proved that $\left(\bar{v}_{\perp}^{0}, \ldots, \bar{v}_{k}^{0}, \bar{u}^{0}\right)$ satisfies the constrained saddle point for $\bar{u}^{\circ} \geq \overline{0}, \bar{v}^{0} \in V=$ $[\bar{v} \mid \bar{g}(\bar{v}) \geq \bar{o}, \bar{v} \geq 0]$ if the following optimality conditions are met: 
C. 1

(i) $\bar{v}^{0} \operatorname{maximizes} \Phi(v)+\bar{u}^{\prime} \bar{g}(\bar{v})$ over $v$

(ii) $\left(\bar{u}^{0}\right)^{\prime} \bar{g}\left(\vec{v}^{0}\right)=0$

(iii) $\overline{\mathrm{u}}^{0} \geqslant \overline{\mathrm{o}}$

(iv) $\overline{\mathrm{g}}\left(\overline{\mathrm{v}}^{0}\right) \geq \overline{\mathrm{o}}$

The vector $\overline{\mathrm{u}}$ is called an optimal Lagrange multiplier vector, a dual vector or a "shadow price" vector.

The proof of the equivalency of the saddle point problem and the original optimization problem is given in the following theorem.

Theorem 3.9 . If $\left(\bar{v}_{1}^{0}, \ldots, \bar{v}_{k}^{0}, \vec{u}^{0}\right)$ is a saddle point of the Lagrangean Function in the domain $\vec{v} \in V, \bar{u}^{\circ} \geqslant \bar{o}$, then $\left(\bar{v}_{1}^{0}, \ldots, \bar{v}_{k}^{0}\right)=\bar{v}^{0}$ is an optimum solution of the Central System problem and meets the optimality conditions (i) - (iv).

Proof: According to the definition of a saddle point we have

$$
\begin{aligned}
& \Phi(\bar{v})+\left(\bar{u}^{0}\right)^{\prime} \bar{g}(\bar{v}) \leq \Phi\left(\bar{v}^{0}\right)+\left(\bar{u}^{0}\right)^{\prime} \bar{g}\left(\bar{v}^{0}\right) \leq \\
& \Phi\left(\bar{v}^{0}\right)+(\bar{u})^{\prime} \bar{g}\left(\bar{v}^{0}\right)
\end{aligned}
$$

The right-hand inequality holds for any $\bar{u} \supseteq \overline{0}$, therefore

$$
\bar{g}\left(\bar{v}^{0}\right) \geqslant \overline{0}
$$

and

$$
\left(\bar{u}^{0}\right)^{\prime} \bar{g}\left(\bar{v}^{0}\right)=0
$$

Then the left-hand inequality becomes

$$
\Phi(v)+\left(\bar{u}^{0}\right)^{\prime} \bar{g}(\bar{v}) \leq \Phi\left(\bar{v}^{0}\right) \text { for } \bar{v} \in v
$$


Since $V=\left[\begin{array}{l|l}\bar{v} & \bar{g}(\bar{v}) \geq \bar{o}, \bar{v} \geq \bar{o}\end{array}\right]$ and any $\bar{u} \geq \overline{0}$, then for $\bar{v}$ to be an element of $\mathrm{V}$ tre should have

$$
(\overline{\mathrm{u}})^{\prime} \overline{\mathrm{g}}(\overline{\mathrm{v}}) \geq 0
$$

then

$$
\phi(\bar{v}) \leq \phi\left(\bar{v}^{\circ}\right) \text { for all } \bar{v} \in V
$$

a result which proves that $\vec{v}^{0}$ is optimal for the central problem. The optimality conditions are met of course by the relations (3.68) $(3.70)$.

The converse of the above theorem holds only if the Central System problem meets certain regularity assumptions. These are:

(i) The objective function $\phi(\bar{v})$ is concave and the constraint set $\bar{g}(\bar{v}) \geq \bar{o}$ is convex

(ii) The constraints satisfy Slater's condition (64) which is: there must exist a vector $\bar{v}^{*}$ for which $\mathrm{g}_{i}\left(\overline{\mathrm{v}}^{*}\right)>0, \quad i=1, \ldots, \mathrm{m}_{\mathrm{o}}$

According to Theorem 3.7, $\Phi(\tilde{v})$ is concave and since the constraint set $\bar{g}(\bar{v}) \geqslant \bar{o}$ consists of linear equations it is convex. Also it can be easily shown that Slater's condition can be met by the Central System problem since the constraints are linear. Therefore the Central System problem meets all the regularity assumptions and therefore the converse theorem which follows holds.

Theorem 3.10. (Kuhn-Tucker) The vector $\bar{v}^{0}$ is optimal if and 
only if there exists a vector $\bar{u}^{0} \geq \overline{0}$ such that $\left(\bar{v}^{0}, \vec{u}^{0}\right)$ is the saddle point of the function $L(\bar{v}, \bar{u})$. The proof can be found in (63). The optimality conditions C.1 apart from clarifying some of the properties of a saddle point do not contribute to an actual method for solving the general nonlinear programming problem.

Kuhn and Tucker improved the optimality conditions by assuming first differentiability of all the functions in the system and second accepting the regularity assumptions. Their result is the following theorem.

Theorem 3.11. Assume the Central System problem is differentiable and meets the regularity assumptions, then the following conditions hold.

$$
\begin{aligned}
& \text { (i) } \nabla \Phi(v)+\sum_{i=1}^{i=m_{0}} u_{i}^{0} \quad \nabla g_{i}\left(\bar{v}^{0}\right)=0 \\
& \text { (ii) }\left(\bar{u}^{\circ}\right)^{\prime} \tilde{g}\left(\bar{v}^{0}\right)=0 \\
& \text { (iii) } \bar{u}^{0} \geq \bar{o} \\
& \text { (iv) } \bar{g}\left(\bar{v}^{0}\right) \geq \overline{0}
\end{aligned}
$$

If the constraint set contains equalities then condition (iii) is valid only for the inequalities since the dual variables of the equalities are unrestricted. Also condition (ii) applies only for the inequality constraints and condition (iv) in the case of equalities

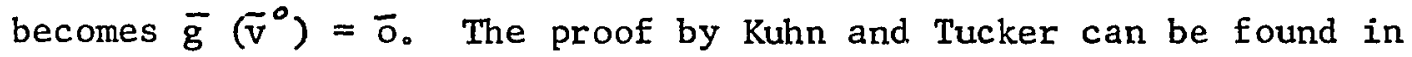
(93). 
For equality constraints the conditions C. 2 reduce the general differentiable nonlinear programming problem to that of finding the solution of a system of equations thus rendering its solution easy to achieve. But the picture is not so clear for inequality or mixed constraints, apart from the fact that they have been used as a base of development of certain algorithms and as stopping criteria.

Unfortunately, the Kuhn-Tucker conditions C.2 cannot be applied to the Central System problem since the objective function $\Phi(\bar{v})$ is not differentiable. Therefore, new optimality conditions have to be developed for the Central System problem, based on the properties of the function $\Phi(\bar{v})$.

Development of the Centra1 Problem Optimality Conditions

The optimality conditions for the Central System problem will be developed by utilizing some of the previously-proven results for the subsystems and the Central System in combination with new theorems developed in this section. In general the approach will utilize throughout direction vectors in the real space.

Theorem 3.12. The directional derivative of the function $\phi_{e}\left(\bar{v}_{e}\right)$ is equa1 to $\quad \hat{\bar{w}}_{f}^{0}\left(\bar{v}_{e}\right)^{\prime} \bar{d}_{e}$ where $\cdots \hat{\bar{w}}_{e}^{0}\left(\bar{v}_{e}\right)$ is the vector minimizing $\overline{\mathrm{w}}_{e}^{0}\left(\overline{\mathrm{v}}_{e}\right)^{\prime} \overline{\mathrm{d}}_{e}$ over the set $\mathrm{w}_{e}\left(\overline{\mathrm{v}}_{e}\right)$ for any $\overline{\mathrm{d}}_{e}$, in other words

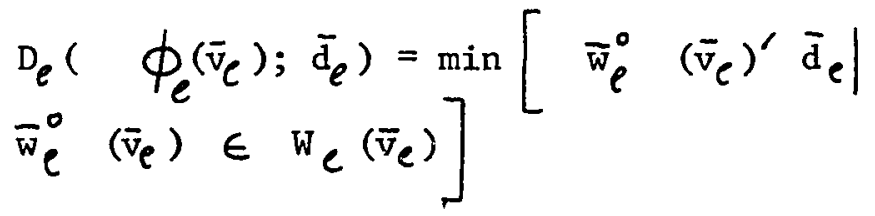

Proof: From Theorem 3.4 and relation (3.53) we have 
54

$$
\begin{aligned}
& \bar{w}_{e}^{0}\left(\bar{v}_{e}^{*}\right)^{\prime}\left(\bar{v}_{e}^{k}-\bar{v}_{e}^{*}\right) \leq \phi_{e}\left(\bar{v}_{e}^{k}\right)-\phi_{e}\left(\bar{v}_{e}^{*}\right) \leq \\
& \bar{w}^{0}\left(\bar{v}_{e}^{k}\right)^{\prime}\left(\bar{v}_{e}^{-}-\bar{v}_{e}^{k}\right)
\end{aligned}
$$

for $\bar{v}_{e}^{K} \leq \bar{v}_{e}^{\circ}$ and $\bar{w}_{e}^{0}\left(\bar{v}_{l}^{*}\right) \leq \bar{w}_{e}^{0}\left(\bar{v}_{e}^{k}\right)$

then increase the vector $\bar{v}_{e}^{k}$ as follows

$$
\overline{\mathrm{v}}_{e}^{\mathrm{k}}=\overline{\mathrm{v}}_{e}^{0}+\mathrm{s}_{e}, \mathrm{~s}>0
$$

Now, if we substitute (3.74) into (3.72) we have

$$
\begin{aligned}
& s \bar{w}^{0}\left(\bar{v}_{e}^{*}\right)^{\prime} \bar{d}_{e} \leq \phi_{e}\left(\bar{v}_{e}^{*}+s \bar{d}_{e}\right)-\phi_{e}\left(\bar{v}_{e}^{*}\right) \leq \\
& s \bar{w}^{0}\left(\bar{v}_{e}^{k}\right)^{\prime}\left(-\bar{d}_{e}\right)
\end{aligned}
$$

Now dividing (3.75) by $s>0$ we find

$$
\begin{aligned}
& \overline{\mathrm{w}}^{0}\left(\overline{\mathrm{v}}_{e}^{\cdot}\right)^{\prime} \overline{\mathrm{d}}_{e} \leq \frac{\phi_{e}\left(\overline{\mathrm{v}}_{e}^{*}+s \overline{\mathrm{d}}_{e}\right)-\phi_{e}\left(\overline{\mathrm{v}}_{e}^{*}\right)}{\mathrm{s}} \leq \\
& -\overline{\mathrm{w}}^{0}\left(\overline{\mathrm{v}}_{e}^{\mathrm{k}}\right)^{\prime} \overline{\mathrm{d}}_{e}
\end{aligned}
$$

We can select $\bar{w}^{\circ}\left(\bar{v}_{e}^{\circ}\right) \in W_{e}\left(\bar{v}_{e}^{\cdot}\right)$ such that

$$
\begin{gathered}
\min \left\{\overline{\mathrm{w}}_{e}^{0}\left(\overline{\mathrm{v}}_{e}\right)^{\prime} \overline{\mathrm{d}}_{e} \mid \overline{\mathrm{w}}_{e}{ }^{\circ}\left(\overline{\mathrm{v}}_{e}\right) \in \mathrm{w}_{e}\left(\overline{\mathrm{v}}_{e}\right)\right\}= \\
\left\{\overline{\mathrm{w}}^{\circ}\left(\overline{\mathrm{v}}_{e}\right)^{\prime} \overline{\mathrm{d}}_{e} \mid \overline{\mathrm{w}}^{0}\left(\overline{\mathrm{v}}_{e}^{-}\right) \in \mathrm{w}_{e}\left(\overline{\mathrm{v}}_{e}^{\circ}\right)\right.
\end{gathered}
$$

and thus

$$
\begin{aligned}
& \min \left(\bar{w}_{e}^{0}\left(\bar{v}_{e}\right)^{\prime} \bar{d}_{e}\right) \leq \frac{\phi_{e}\left(\bar{v}_{e}+s \bar{d}_{e}\right)-\dot{\phi}_{e}\left(\bar{v}_{e}\right)}{s} \leq \\
& -\bar{w}^{0}\left(\bar{v}_{e}^{k}\right)^{\prime} \bar{d}_{e} \mid \bar{w}_{e}^{0}\left(\bar{v}_{e}\right) \in \mathrm{w}\left(\bar{v}_{e}\right)
\end{aligned}
$$


and

$$
\begin{aligned}
& -\bar{w}_{e}^{o}\left(\bar{v}_{e}^{k}\right)^{\prime} \bar{d}_{e} \leq \max \left\{-\bar{w}_{e}^{0}\left(\bar{v}_{e}^{k}\right)^{\prime} \bar{d}_{e} \mid\right. \\
& \left.\bar{w}_{e}^{0}\left(\bar{v}_{e}^{k}\right) \in w_{e}\left(\bar{v}_{e}^{k}\right)\right\}
\end{aligned}
$$

and since

$$
\begin{aligned}
& \max \left\{-\overline{\mathrm{w}}_{e}{ }_{e}\left(\overline{\mathrm{v}}_{e}^{k}\right)^{\prime} \overline{\mathrm{d}}_{e} \mid \overline{\mathrm{w}}_{e}^{0}\left(\overline{\mathrm{v}}_{e}^{k}\right) \in \mathrm{w}_{e}\left(\overline{\mathrm{v}}_{e}^{k}\right)\right\}= \\
& \min \left\{\overline{\mathrm{w}}_{e}{ }_{e}\left(\overline{\mathrm{v}}_{e}^{k}\right)^{\prime} \overline{\mathrm{d}}_{e} \mid \overline{\mathrm{w}}_{e}{ }_{e}\left(\overline{\mathrm{v}}_{e}^{k}\right) \in \mathrm{w}_{e}\left(\overline{\mathrm{v}}_{e}^{k}\right)\right.
\end{aligned}
$$

we have

$$
\begin{aligned}
& -\overline{\mathrm{w}}_{e}^{0}\left(\overline{\mathrm{v}}_{e}^{k}\right)^{\prime} \overline{\mathrm{d}}_{e} \leq \min \left\{\overline{\mathrm{w}}_{e}^{0}\left(\overline{\mathrm{v}}_{e}^{k}\right)^{\prime} \overline{\mathrm{d}}_{e} \mid\right. \\
& \overline{\mathrm{w}}_{e}^{0}\left(\overline{\mathrm{v}}_{e}^{k}\right) \in \mathrm{w}_{e}\left(\overline{\mathrm{v}}_{e}^{k}\right)
\end{aligned}
$$

Now, let us find the effect of reducing $\vec{v}_{e}^{k}$ to $\bar{v}_{e}^{*}$

$$
\overline{\mathrm{w}}_{e}^{0}\left(\overline{\mathrm{v}}_{e}^{*}\right) \in \overline{\mathrm{w}}_{e}\left(\overline{\mathrm{v}}_{e}^{*}\right)
$$

from Theorem 3.3, for

$$
\begin{aligned}
& \lim \bar{w}_{l}^{0}\left(\bar{v}_{l}^{k}\right)=\bar{w}_{e}^{0}\left(\bar{v}_{l}^{0}\right) \\
& \bar{v}_{e}^{k} \rightarrow \bar{v}_{l}^{0}
\end{aligned}
$$

wiose limit $\bar{W}_{e}^{\circ}\left(\bar{v}^{*}\right)$ exists, since $W_{e}\left(\vec{v}_{e}^{*}\right)$ is bounded. Now since $\oint_{e}\left(\bar{v}_{e}\right)$ is concave and $\vec{v}^{k} \geq \vec{v}^{\bullet}$ we have

$$
\bar{w}_{\rho}^{0}\left(\bar{v}^{*}\right) \leq \bar{w}_{e}^{0}\left(\bar{v}^{k}\right)
$$

and when multiplied by $+\overline{\mathrm{d}}_{e}$ we have

$$
+\overline{\mathrm{w}}_{e}^{0}\left(\overline{\mathrm{v}}^{*}\right)^{\prime} \overline{\mathrm{d}}_{e} \geq+\overline{\mathrm{w}}_{e}^{0}\left(\overline{\mathrm{v}}^{\mathrm{k}}\right) \overline{\mathrm{d}}_{e},\left(\overline{\mathrm{d}}_{\bar{l}} \text { negative }\right)
$$


Now, by the same arguments as in (3.79) - (3.80) we have

$$
\begin{aligned}
& \min \left\{\bar{w}_{e}^{0}\left(\bar{v}_{e}^{*}\right)^{\prime} \overline{\mathrm{d}}_{e} \mid \overline{\mathrm{w}}_{e}^{0}\left(\overline{\mathrm{v}}_{e}^{*}\right) \in \mathrm{w}_{e}\left(\overline{\mathrm{v}}_{e}^{*}\right)\right\} \geq \\
& \min \left\{\overline{\mathrm{w}}_{e}^{0}\left(\overline{\mathrm{v}}_{e}^{k}\right)^{\prime} \overline{\mathrm{d}}_{e} \mid \overline{\mathrm{w}}_{e}^{0}\left(\overline{\mathrm{v}}_{e}^{k}\right) \in \mathrm{w}_{e}\left(\overline{\mathrm{v}}_{e}^{k}\right)\right\}
\end{aligned}
$$

Therefore, from (3.86), (3.77) and (3.78) we have

$$
\begin{aligned}
& {\left[\begin{array}{l}
\text { minimum }\left(\bar{w}_{e}^{0}\left(\bar{v}_{e}\right)^{\prime} \bar{d}_{l}\right) \\
\bar{w}_{e}^{0}\left(\bar{v}_{e}\right) \in w_{e}\left(\bar{v}_{e}\right)
\end{array}\right] \leqslant \frac{\phi_{e}\left(\bar{v}_{e}^{*}+s \bar{d}_{e}\right)-\phi_{e}\left(\bar{v}_{e}^{*}\right)}{s} \leqslant}
\end{aligned}
$$

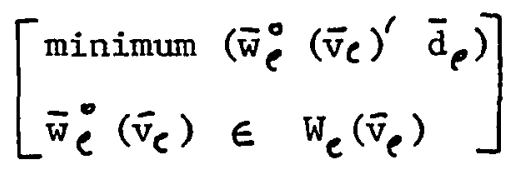

which implies

$$
\begin{aligned}
& \frac{\phi_{e}\left(\bar{v}_{e}+s \bar{d}_{e}\right)-\phi_{e}\left(\bar{v}_{e}\right)}{s}=\min \left\{\bar{w}_{e}^{0}\left(\bar{v}_{e}\right)^{\prime} \bar{d}_{e} \mid\right. \\
& \left.\bar{w}_{e}^{0}\left(\bar{v}_{e}\right) \in w_{e}\left(\bar{v}_{e}\right)\right\}
\end{aligned}
$$

and after taking the limit $S \rightarrow 0$ we have

$$
\begin{aligned}
\mathrm{D}_{e}\left(\phi_{e}\left(\overline{\mathrm{v}}_{e}\right) ; \overline{\mathrm{d}}_{e}\right)= & \operatorname{minimum}\left(\overline{\mathrm{w}}_{e}^{\circ}\left(\overline{\mathrm{v}}_{e}\right)^{\prime} \overline{\mathrm{d}}_{e}\right) \\
& \overline{\mathrm{w}}_{e}^{0}\left(\overline{\mathrm{v}}_{e}\right) \in \mathrm{w}_{e}\left(\overline{\mathrm{v}}_{e}\right)
\end{aligned}
$$

which proves the theorem.

Now, since Centra! System objective function (3.63) is concave its directional derivative exists everywhere by Theorem 3.8 . Therefore we have 


$$
\begin{aligned}
& \lim _{s \rightarrow 0} \frac{\Phi_{(\bar{v}+s \bar{d})}-\Phi(\bar{v})}{s}=\lim _{s \rightarrow 0} \frac{\sum_{l=1}^{e=k} \phi_{e}\left(\bar{v}_{e}+s \bar{d}_{e}\right)-\sum_{l=1}^{l=k} \phi_{e}^{\left(\bar{v}_{e}\right)}}{s} \\
& =\lim _{S \rightarrow 0} \frac{\sum_{l=1}^{l=k}\left(\phi_{e}\left(\bar{v}_{e}+s \bar{d}_{e}\right)-\phi_{e}\left(\bar{v}_{l}\right)\right)}{s} \\
& =\sum_{e=1}^{\ell_{2 k}} D_{e}\left(\phi_{e}\left(\bar{v}_{e}\right) ; \bar{d}_{e}\right) \\
& =\sum_{e=1}^{e=k} \bar{w}_{e}^{m}\left(\bar{v}_{e}\right)^{\prime} \bar{d}_{e} \\
& =\left(\bar{w}^{m}\right)^{\prime} \bar{d}
\end{aligned}
$$

where we went from (3.89) to (3.90) by formula (3.88) assuming that we found the minima in (3.88) and

$$
\overline{\mathrm{d}}=\left[\begin{array}{c}
\overline{\mathrm{d}}_{1} \\
\overline{\mathrm{d}}_{2} \\
\vdots \\
\overline{\mathrm{d}}_{k}
\end{array}\right]\|\overline{\mathrm{d}}\|=1, \overline{\mathrm{w}}^{m} \equiv\left[\begin{array}{c}
\overline{\mathrm{w}}_{1}^{m}\left(\overline{\mathrm{v}}_{1}\right) \\
\overline{\mathrm{w}}_{2}^{m}\left(\overline{\mathrm{v}}_{2}\right) \\
\vdots \\
\vdots \\
\overline{\mathrm{w}}_{k}^{m}\left(\overline{\mathrm{v}}_{k}\right)
\end{array}\right], \overline{\mathrm{w}}_{\mathrm{e}}^{m}\left(\overline{\mathrm{v}}_{\mathrm{e}}\right) \in \mathrm{R}^{m_{0}}
$$

where $\bar{w}_{e}^{m}\left(\bar{v}_{e}\right)^{\prime} \bar{d}_{e}=\min \left\{\bar{w}_{e}^{0}\left(\bar{v}_{e}\right)^{\prime} \bar{d}_{e} \mid \bar{w}_{e}\left(\bar{v}_{e}\right) \in w_{e}\left(\bar{v}_{e}\right)\right\}$ 
The set of all the feasible direction vectors at a given

$$
\begin{aligned}
\bar{v} \in V & =\{\bar{v} \mid \bar{g}(\bar{v}) \geq \bar{o}, \bar{v} \geq \bar{o}\} \quad \text { will be denoted by } \\
F(\bar{v}) & =\{\bar{d} \mid \bar{v}+s \bar{d} \in V, a \text { exists such that } \\
\sigma & \geq s \geq 0\}
\end{aligned}
$$

and a feasible direction will be usable if

$$
\lim _{s \rightarrow 0} \frac{\Phi(\bar{v}+s \bar{d})-\Phi(\bar{v})}{s}=\left(\bar{w}^{m}\right)^{\prime} \bar{d}>0
$$

holds.

Since by assumption $S>0$ we have for a usable direction

$$
\Phi(\bar{v}+s \bar{d}) \geq \Phi(\bar{v})
$$

Theorem 3.13. If there is no usable feasible direction at the point $\overline{\mathrm{v}}^{\mathrm{k}} \in \mathrm{V}$ then $\overline{\mathrm{v}}^{\mathrm{k}}$ is an optimum vector $\overrightarrow{\mathrm{v}}^{\circ} \in \mathrm{V}$ of the function $\phi(\bar{v})$.

Proof: Assume that there exists a $\bar{v}^{0} \in V$ such that $\bar{v}^{\bullet}>\bar{v}^{k}$ and by concavity $\left.\Phi\left(\bar{v}^{*}\right)\right\rangle \phi\left(\bar{v}^{k}\right)$ then by the right inequality of (3.72) we have

$$
\Phi\left(\bar{v}^{k}\right)-\Phi\left(\bar{v}^{*}\right)<\bar{w}^{0}\left(\bar{v}^{k}\right)^{\prime}\left(\bar{v}^{*}-\bar{v}^{k}\right)
$$

and since $\overline{\mathrm{v}}^{-}>\overrightarrow{\mathrm{v}}^{\mathrm{k}}$

$$
\left(\bar{w}^{m}\right)^{\prime}\left(\bar{v}^{\bullet}-\bar{v}^{k}\right)>0
$$

is true, and thus $\left(\bar{v}^{*}-\vec{v}^{k}\right)$ is a usable feasible direction. 
Therefore, if a usable feasible direction does not exist

$$
\left(\bar{w}^{m}\right)^{\prime}\left(\bar{v}^{*}-\bar{v}^{k}\right) \ngtr 0
$$

and $\bar{v}^{0}=\bar{v}^{*}=\bar{v}^{k}$ and therefore $\bar{v}^{k}$ maximizes $\Phi(v)$ on $v$.

Now let $\overline{\mathrm{v}}^{\bullet}=\overline{\mathrm{v}}^{0}+\overline{\mathrm{d}}, \overline{\mathrm{d}} \in F\left(\overline{\mathrm{v}}^{0}\right)$

by $(3.99)$

$$
\left(\bar{w}^{m}\right)^{\prime} \overline{\mathrm{d}} \ngtr 0
$$

or

$$
\left(\bar{w}^{m}\right)^{\prime} \overline{\mathrm{d}} \leqslant 0 \text { for a11 } \overrightarrow{\mathrm{d}} \in F\left(\overline{\mathrm{v}}^{\circ}\right)
$$

which means that if $\overrightarrow{\mathrm{v}}^{0}$ is optimal for the concave Central System problem, then (3.100) holds. Also

$$
\left(\bar{w}^{m}\right)^{\prime} \bar{d} \leq 0 \text { for a } 11 \bar{d} \in \hat{F}\left(\bar{v}^{\circ}\right)
$$

because if $\bar{d}^{-k} \in F\left(\bar{v}^{0}\right)$ by $(3.100)$

$$
\left(\bar{w}^{m}\right)^{\prime} \overline{\mathrm{d}}^{k} \leq 0 \text { for a11 k }
$$

and if the limit is taken

$$
\lim _{k \rightarrow \infty}\left(\bar{w}^{m}\right)^{\prime} \bar{d}^{k}=\left(\bar{w}^{m}\right) \quad \bar{d}^{\infty} \leqslant 0
$$

and since $\bar{d}^{-\infty}$ is any point in $\hat{F}\left(\bar{v}^{0}\right),(3.101)$ holds.

Theorem 3.14 (Farka's Lemma). The statement

$$
\bar{c}^{\prime} \bar{x} \leq 0
$$


for a11 $\bar{x}$ such that

$A \bar{x} \geq \bar{o}$

is equivalent to the statement that there exists a $\overline{\mathrm{u}} \geqslant \overline{\mathrm{o}}$ such that

$$
\bar{c}+A^{\prime} \bar{u}=\bar{o}
$$

The proof is given in Simonnard (37).

The constraint set ( 3.64 ) may be divided into two sets, the active set and the inactive set for any feasible vector $\bar{v} \in V$. These are defined as follows:

$$
\begin{aligned}
& \text { Active set } A(\bar{v})=\left\{\begin{array}{l|l}
i & g_{i}(\bar{v})=0
\end{array}\right\} \\
& \text { Inactive set } I(\bar{v})=\left\{\begin{array}{l|l}
i \mid & \left.g_{i}(\bar{v})\right\rangle
\end{array}\right\}
\end{aligned}
$$

We need this disection because only the active set needs to be considered for formulating the optimality conditions because any small movement in any direction in an inactive constraint does not violate the constraint, and therefore the inactive constraints are not needed in our analysis.

In order to formulate the optimality conditions we need to formulate the set $\hat{F}(\bar{v})$ in terms of the active Central system constraints. For that purpose we define the set

$$
\underline{O}(\bar{v})=\left\{\overline{\mathrm{d}} \mid \nabla \mathrm{g}_{i}(\overline{\mathrm{v}})^{\prime} \overline{\mathrm{d}} \geq 0, \quad i \in \mathrm{A}(\overline{\mathrm{v}})\right\} \quad \text { (3.104) }
$$

The theorem that follows proves that $\hat{F}(\bar{v}) \quad C \quad \underline{O}(\mathrm{v})$. 
Theorem 3.15. The closure set $\hat{F}(\bar{v})$ of the set $F(\bar{v})$ of all feasible directions is contained in the set $\underline{O}(\bar{v})$ of all the feasible directions expressed in terms of the active constraints, i.e. $\hat{F}(\bar{v})=\underline{O}(\bar{v})$.

Proof: Let $\overline{\mathrm{d}} \in F(\overline{\mathrm{v}})$ for $\mathrm{g}_{i}(\overline{\mathrm{v}})=0$. Then if $\nabla g_{i}(\bar{v})^{\prime} \bar{d}<0$, for small s we have

$$
g_{i}(\bar{v}+s \bar{d})<g_{i}(\bar{v})=0
$$

which shows that such $\tilde{d}$ is not feasible and therefore for feasibility

$$
\nabla g_{i}(\bar{v})^{\prime} \bar{d} \geq 0
$$

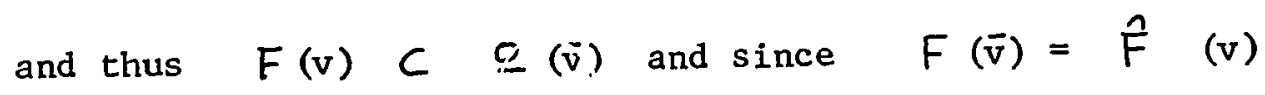

$$
\begin{aligned}
& \hat{F}(\bar{v}) \subset \underline{O}(\bar{v}) \\
& \text { Now; let } \overline{\mathrm{d}} \in \underline{O}(\overline{\mathrm{v}}) \text { for } \nabla g_{i}(\overline{\mathrm{v}})^{\prime} \overline{\mathrm{d}} \geq 0, i \in A(\overline{\mathrm{v}}) \\
& \text { and assume that } \overline{\mathrm{v}}+\mathrm{s} \overline{\mathrm{d}} \notin \mathrm{V} \text { then }
\end{aligned}
$$

$$
g_{i}(\bar{v}+s \bar{d})<0
$$

and

$$
\nabla g_{i}(\bar{v}+s \bar{d}) \bar{d}<0
$$

which clearly contradicts the assumption and therefore $\bar{v}+s \bar{d} \in \quad v$ or $\underline{Q}(\bar{v}) \subset \hat{F}(\bar{v})$ and thus

$$
\hat{F}(v)=Q(\bar{v})
$$


The existence of feasible directions in $\underline{Q}(\bar{v})$ is assured by Slater's condition that there exist a vector $\vec{v}^{*}$ such that $\bar{g}\left(\bar{v}^{r}\right)>\bar{o}$

Now we can reformulate one of the results of Theorem 3.13 as Theorem 3.16 .

Theorem 3.16. If $\overrightarrow{\mathrm{v}}^{\circ}$ is optimal for the concave Central system problem, then

$$
\left(\bar{w}^{m}\right)^{\prime} \vec{d} \leq 0
$$

for a11

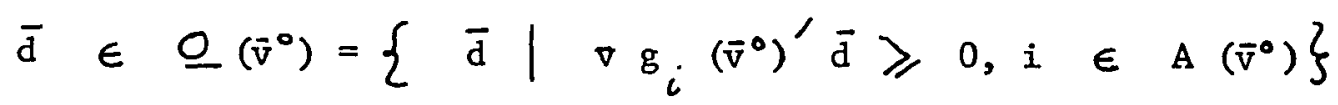

The statement of the theorem above is by Theorem 3.14 equivalent to the statement that there exist multipliers $u_{i}^{0} \geqslant 0$ such that

$$
\bar{w}^{m}+\sum_{i=1}^{i=m_{0}} \ddot{u}_{i}^{0} \nabla g_{i}\left(\bar{v}^{0}\right)=0
$$

Then the optimality conditions for the Central system problem are given by the following theorem.

Theorem 3.17. Consider the Central System problem as given by $(3.63)-(3.66)$. The following conditions define its optimality: 
$\mid \begin{aligned} & \text { (i) } \overline{\mathrm{w}}^{m}+\sum_{i=1}^{i=m_{0}} u_{i}^{0} \nabla g_{i}\left(\bar{v}^{0}\right)=0 \\ & \text { (ii) }\left(\overline{\mathrm{u}}^{0}\right)^{\prime} \overline{\mathrm{g}}\left(\overline{\mathrm{v}}^{0}\right)=0 \\ & \text { (iii) } \overline{\mathrm{u}}^{0} \geq \overline{\mathrm{o}} \\ & \text { (iv) } \overline{\mathrm{g}}\left(\overline{\mathrm{v}}^{0}\right) \Rightarrow \overline{\mathrm{o}}\end{aligned}$

Proof: Condition (iv) is the feasibility condition; (iii) is from the Farka's Lemma. Condition (ii) is the complementarity condition (3.69) of Theorem 3.9 and (i) is the result (3.107). The optimality conditions C.3 are very important because the vector $\bar{w}^{m}$ of the optimal dual variables of the subsystems is shown to be directly connected with the optimal dual variables of the Central System.

This property will be utilized in the next chapter for the development of the price-resource directive decomposition procedure or coordination method. 


\section{CHAPTER IV}

PRICE-RESOURCE DIRECTIVE COORDINATION: THE ALGORITHM

In this chapter, the basic results developed so far, and especially the optimality conditions C.3, will be expanded towards the creation of a very practical algorithm for the solution of large-scale optimization problems.

The optimality condition $\mathrm{C.} 3(i)$ can be reformulated as follows:

$$
\begin{aligned}
& \overline{\mathrm{w}}^{m}+\sum_{i=1}^{i=m_{0}} \mathrm{u}_{i}^{0} \nabla \mathrm{g}\left(\overline{\mathrm{v}}^{0}\right)=\overline{\mathrm{w}}^{m}+\sum_{i=1}^{i=m_{0}} \mathrm{u}_{i}^{0} \nabla\left[\mathrm{b}_{\alpha_{i}}-\overline{\mathrm{h}}_{i} \overline{\mathrm{v}}^{0}\right] \\
& =\vec{w}^{n}+\sum_{i=1}^{i=m_{0}} u_{i}^{0}\left(-\bar{h}_{i}\right) \\
& =\left[\begin{array}{cc}
\bar{w}_{1}^{m} & \left(\bar{v}_{1}^{0}\right) \\
\bar{w}_{2}^{m} & \left(\bar{v}_{1}^{0}\right) \\
\cdot \\
\cdot \\
\cdot \\
\bar{w}_{k}^{m}\left(\bar{v}_{k}^{0}\right)
\end{array}\right]-H^{\prime} \bar{u}^{0}
\end{aligned}
$$


which means that at the point of optimality

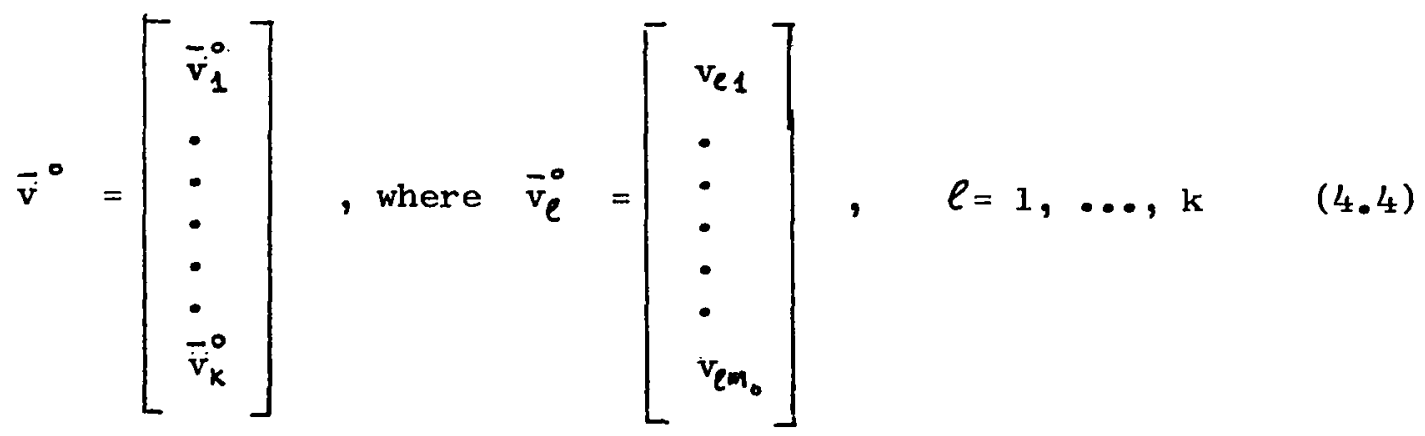

the set of the Central System optimal shadow prices

$$
\vec{u}^{0}=\left[\begin{array}{c}
u_{1}^{0} \\
: \\
\vdots \\
u_{m}^{0}
\end{array}\right]
$$

has to be equal to each set of the minimum optimal shadow prices of the subsystems. For example for subsystem $\ell$ we have

$$
\left[\begin{array}{c}
w_{l 1}^{m} \\
w_{l 2}^{m} \\
\dot{0} \\
w_{l m_{0}}^{m}
\end{array}\right]=\left[\begin{array}{c}
u_{1}^{0} \\
u_{2}^{0} \\
\vdots \\
\cdot \\
u_{m_{0}}^{0}
\end{array}\right], l=1, \ldots, k
$$


and for all the subsystems

$$
\left[\begin{array}{ll}
w_{11}^{m}\left(\bar{v}_{1}\right) \\
w_{12}^{m}\left(\bar{v}_{1}\right) \\
\vdots \\
w_{1 m_{0}}^{m}\left(\bar{v}_{1}\right)
\end{array}\right]=\left[\begin{array}{cc}
w_{21}^{m} & \left(\bar{v}_{2}\right) \\
w_{22}^{m} & \left(\bar{v}_{2}\right) \\
\vdots & \\
\cdot & \\
w_{2 m_{0}}^{m}\left(\bar{v}_{2}\right)
\end{array}\right]=\cdots=\left[\begin{array}{c}
u_{1}^{0} \\
u_{2}^{0} \\
\vdots \\
\vdots \\
u_{m_{0}}^{0}
\end{array}\right]
$$

The result (4.7) is the basis of the Price-Resource Directive Coordination Procedure.

If we assume that the dual variables of the Central System are known, and for an arbitrary partition of the central resources we acquire, after the optimization of the subsystems, sets of subsystem optimal shadow prices, then our objective would be to minimize the mean square deviation of the subsystem shadow prices from the optimal dual variable of the Central System for each resource. In other words we have the problem

$$
\operatorname{minimize} \sum_{\ell=1}^{\ell=k}\left(\bar{w}_{e}^{m}\left(\bar{v}_{e}\right)-\bar{u}^{0}\right)^{2}
$$

$\mathbf{k}$

The minimization of the mean square deviation of the dual variables of the subsystems from the dual variable vector of the Central System presents no problem, since according to the following Theorem the mean of the subsystem shadow prices equals the vector of dual variables for the Central System. 
Theorem 4.1. The mean square deviation of a set of vectors from any vector $\overrightarrow{\mathrm{u}}$ is a minimum if and only if

$\vec{u}^{0}=\sum_{\sum_{E I}{ }^{k=k}}^{\bar{w}_{e}^{m}}\left(\bar{v}_{e}\right)$

$\mathbf{k}$

Proof:

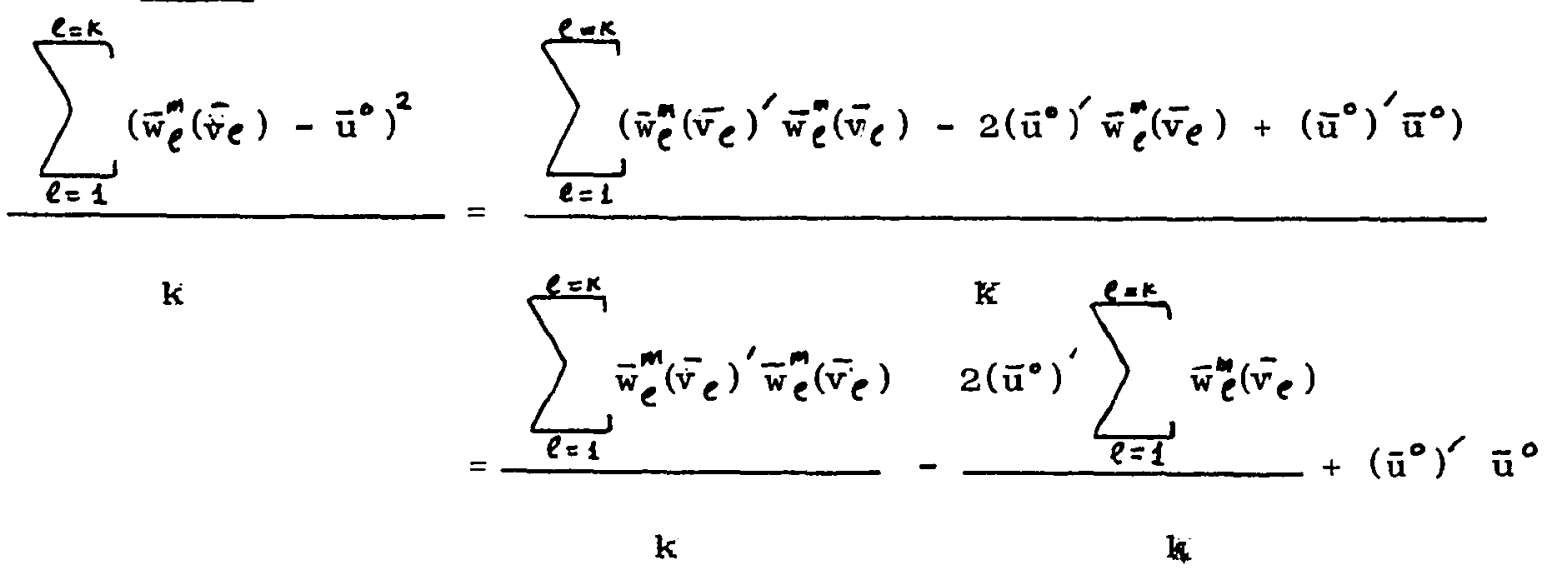

The above, as it can be seen, reaches its minimum value with respect to $\vec{u}^{0}$ when

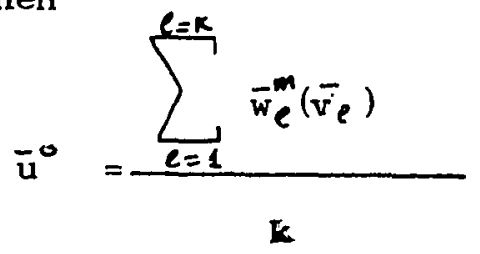

When the mean of the subsystem shadow prices for each resource is found then the deviation from the mean of each subsystem shadow price gives the necessary increase or decrease of the shadow price necessary to bring that price to equality with the dual variable of the Central System. 
As it was shown in Chapter III, the shadow price vector of each subsystem is a step function of the allocated vector of central resources to each subsystem. Therefore, in general, $\bar{w}_{e}^{m}\left(\bar{v}_{e}\right)$ depends nonlinearly on $\bar{v}_{l}$. In the case of relatively small changes in the amounts of resources the rate of change of the shadow price is zero, but we can say that for large changes in the amount of resources the rate of change of the shadow price is approximately negatively proportional to the rate of change of the resources.

From the statements above it becomes clear that the direction of increase or decrease of the amount of resources allocated to a subsystem can be determined from the direction of increase or decrease of the subsystem shadow prices in the process of bringing themselves to equality with the Central System dual variables which were assumed to be known.

I. THE DIRECTION FINDING PROBLEM

Assume an initial partition

$$
\bar{v}_{1}^{1}+\bar{v}_{2}^{1}+\bar{v}_{3}^{1}+\ldots+\bar{v}_{k}^{1} \leqslant \bar{b}_{0}
$$

of the central resources allocated to the $k$ subsystems then we have the 15 optimization problems 


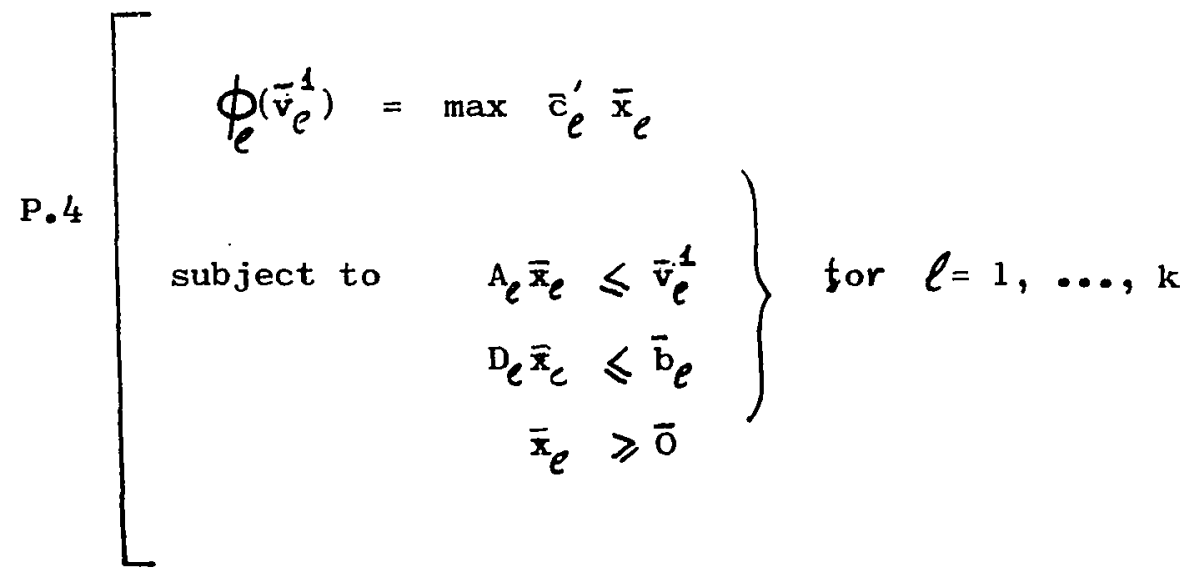

Assume that

$$
\sum_{\ell=1}^{\ell=k} \phi_{l}\left(\bar{v}_{l}^{1}\right) \neq \max \Phi^{(\bar{v})}
$$

then a reallocation is needed which will bring the sum of the optima of the subsystems closer to the required $\max (v)$. This reallocation implies an increase of allocations for some subsystems and a decrease for others. In general we will have by the Definition 3.4 for a direction vector

$$
\left[\begin{array}{c}
\bar{v}_{1}^{1} \\
\bar{v}_{2}^{1} \\
\dot{v_{k}} \\
\bar{v}_{k}^{1}
\end{array}\right]+s\left[\begin{array}{c}
\bar{d}_{1}^{1} \\
\bar{d}_{2}^{1} \\
\dot{0} \\
\bar{d}_{k}^{1}
\end{array}\right]=\left[\begin{array}{c}
\bar{v}_{1}^{2} \\
\bar{v}_{2}^{2} \\
\dot{0} \\
\bar{v}_{k}^{2}
\end{array}\right]
$$

or

$$
\bar{v}^{1}+s \bar{d}^{1}=\bar{v}^{2}
$$


where

$$
\sum_{l=1}^{l=k} \overrightarrow{\vec{v}}_{l}^{2} \leqslant \overrightarrow{\mathrm{b}}_{0}, \quad \overrightarrow{\mathrm{v}}_{e}^{2} \geqslant \overline{0}
$$

and

$$
\bar{d}^{-1}=\text { direction vector changing first partition. }
$$

Then our problem is to find the direction vector which will improve the allocation of the central resources, such that

$$
\sum_{l=1}^{l=k} \phi_{l}\left(\vec{v}_{l}\right) \longrightarrow \max \Phi(\bar{v})
$$

This direction vector has to obey the feasibility constraint (4.13) and in order to improve the allocation it has to be a usable feasible direction vector in other words by formula (3.62) it has to be such that

$$
D(\Phi(\bar{v}) ; \bar{d})>0
$$

or by $(3.91)$

$$
\left(\bar{w}^{m}\right)^{\prime} \bar{d}>0
$$

The direction problem of finding the appropriate $\vec{d}$ which will satisfy (4.16) is solved by considering the optimal dual variables 
which resulted from the solution of problem P. 4 for $\ell=1, \ldots, k$. Let the optimal dual variables of the subsystems for the initial partition of the vector $\bar{b}_{0}$ be

$$
\bar{w}_{1}\left(\bar{v}_{1}^{1}\right), \bar{w}_{2}\left(\bar{v}_{2}^{1}\right), \ldots \ldots, \bar{w}_{k}\left(\bar{v}_{k}^{1}\right)
$$

Since the method empicys successive approximations we assume that the set of optimal dual variabies for each subsystem is a singleton and therefore

$$
\bar{w}_{e}^{m}\left(\bar{v}_{l}\right)=\bar{w}_{e}\left(\bar{v}_{e}\right), \quad \ell=1, \ldots, k
$$

Now, we can use the result of Theorem 4.1 by finding the mean subsystem dual variable for each resource and then substracting each one of these dual variables from the mean to find the required change. Therefore we have

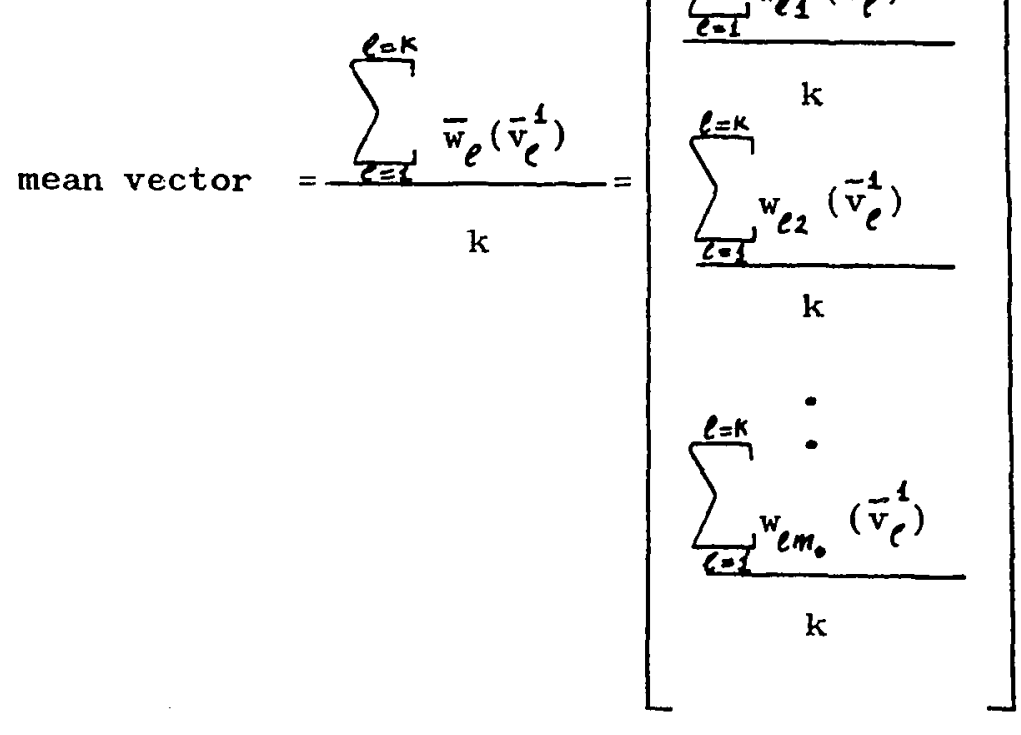


and the change vector

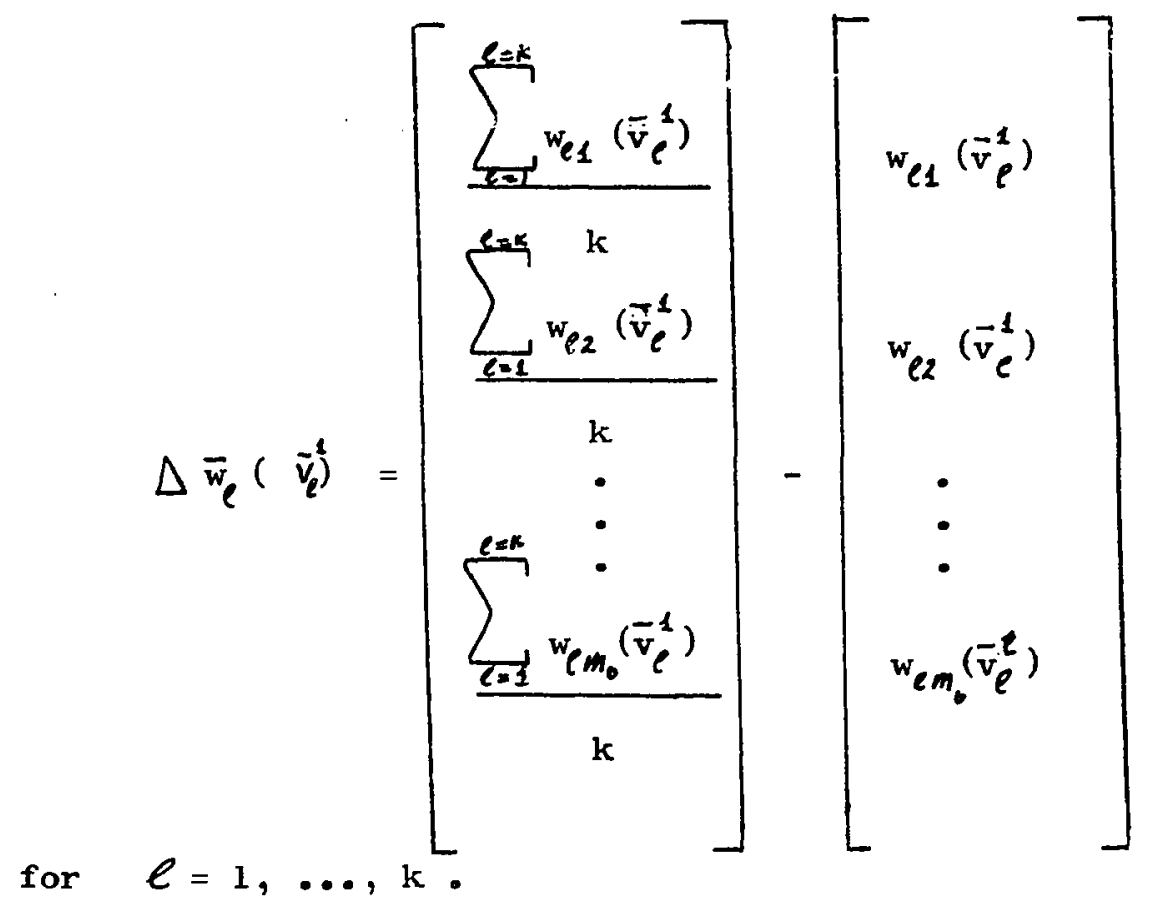

If the means of the subsystems shadow prices are all equal to zero and $\bar{w}_{e}\left(\bar{v}_{\ell}^{1}\right) \geqslant \overline{0}$ for $\ell=1, \ldots, k$ (i.e. all the subsystem constraints which are bounded by the Central System resource vector are weak inequalities), then optimality has been reached for the Central System problem by $(4.7)$, but if $\bar{w}_{e}\left(\bar{v}_{e}^{1}\right)$. is unrestricted for $e=1, \ldots, k$ (i.e. all the subsystem constraints which are bounded by the central System resource vector are equalities), then optimality has been reached only if $\bar{w}_{e}\left(\bar{v}_{e}^{1}\right)=\overline{0}$, for $e=1, \ldots, k$. Now, if the above conditions are not met then we find the change vector for the dual variables of the subsystems using relation (4.20). Then, we are ready to find the proportional change required in the allocation of the centralized resources to conform with the change in the dual variables. Of course we stop if 


$$
\Delta \bar{w}_{e}\left(\bar{v}_{l}^{1}\right)=\bar{o} \text { for all } \ell
$$

since that means that condition (4.7) has been met.

Now to find the proportional change in the allocations we have to consider the percentage change of a subsystem dual variable with respect to the mean subsystem dual variable for one resource and then multiply this ratio by the mean of the partitions for that resource. Therefore we have

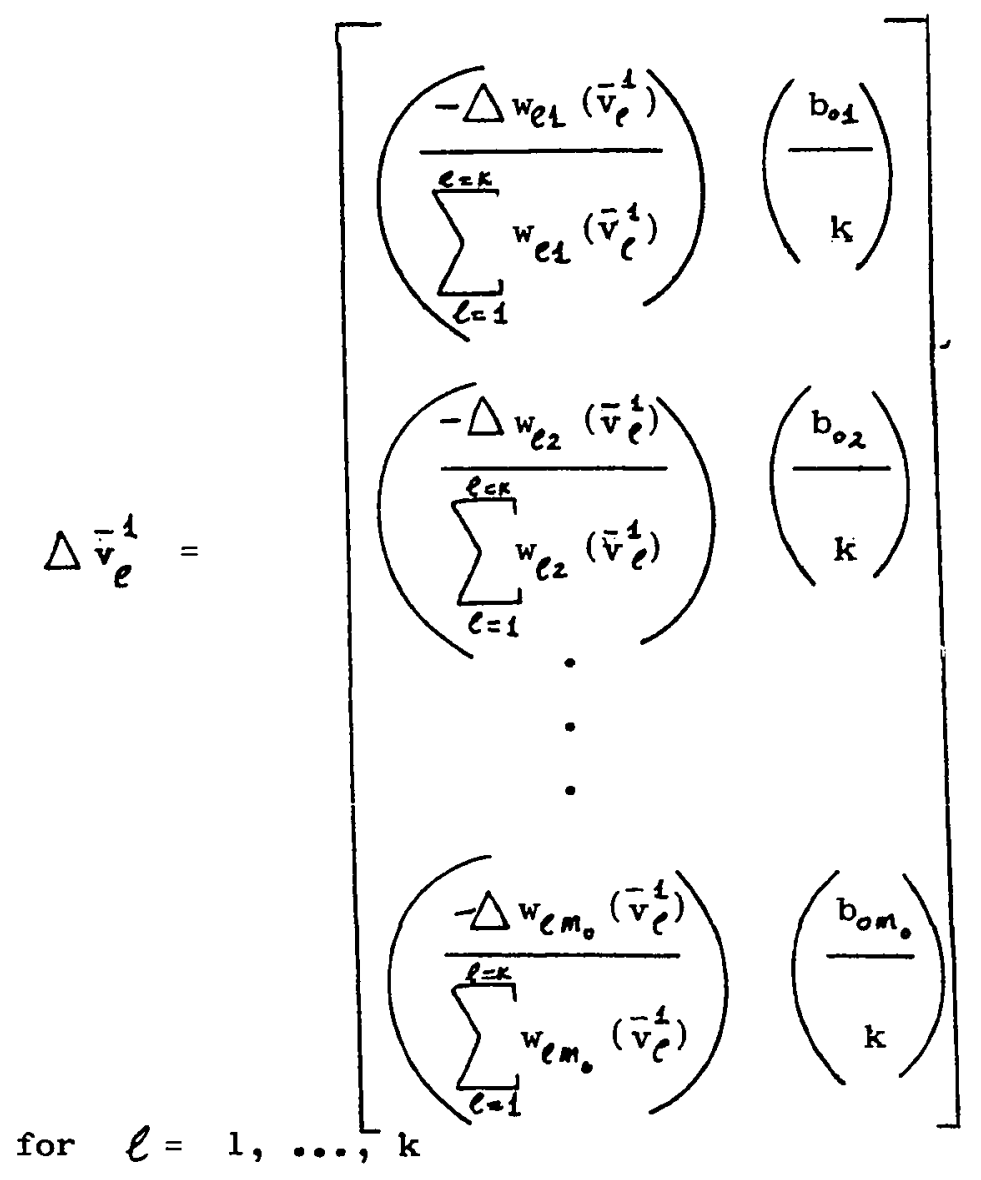

and since the proportional change found could make the allocation infeasible by assigning negative amounts of resources to a sub- 
system if

$$
v_{e 1}^{1}+\Delta v_{e 1}^{1}<0
$$

we have to normalize the changes for the same resource, in other words

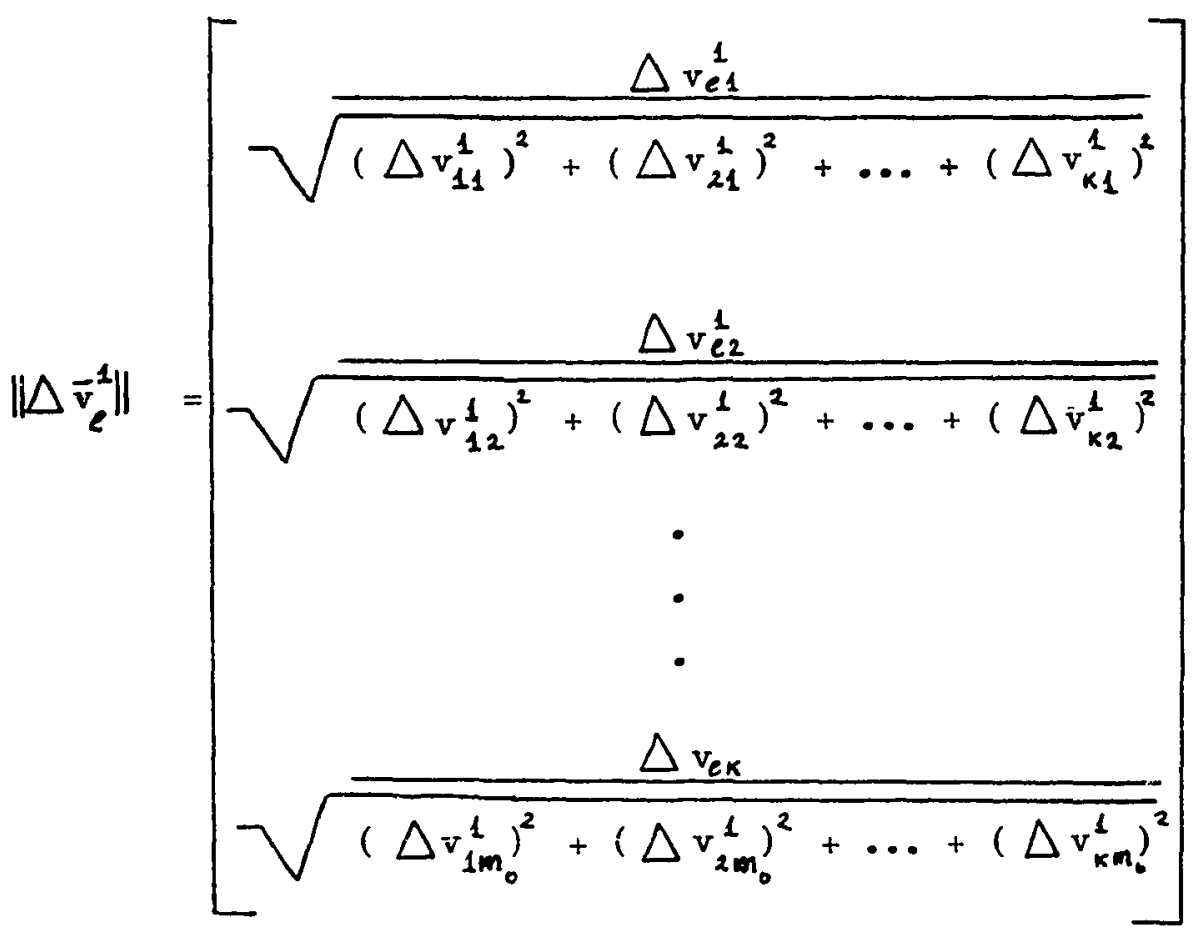

for $\ell=1, \ldots, k, \quad i=1, \ldots, \mathrm{m}_{0}$.

or in matrix form

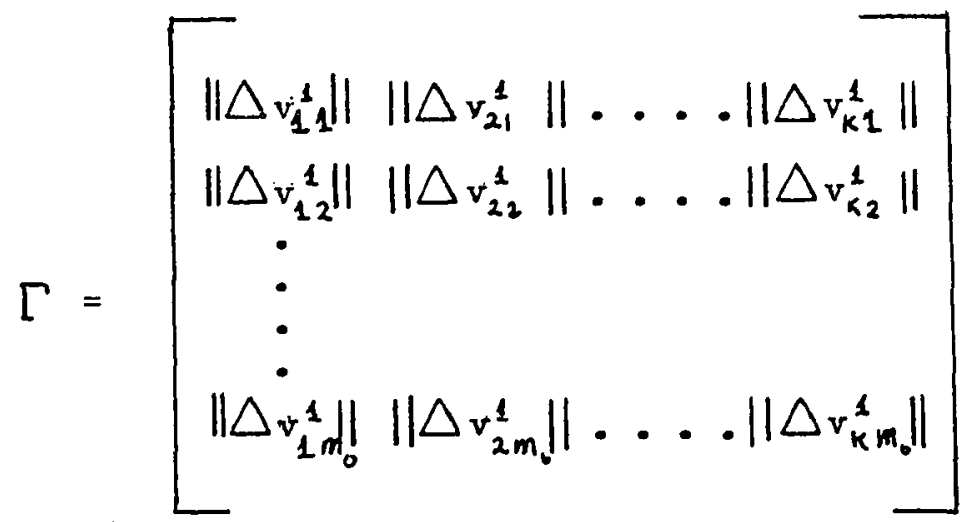


where every row is normalized.

Now in order to find for each resource the maximum change in the indicated direction by the normalized row of the above matrix we have to consider only the negative changes. The new resource allocation $v_{i}^{?}$ which becomes first zero in the relation

$$
v_{e i}^{2}=v_{e i}^{1}+t_{i}\left\|\Delta v_{l i}^{1}\right\|
$$

with an increase in $t_{\ell}$, gives the maximum $t$ that can be used for changing uniformly the allocations. We denote the maximum ${ }^{t} \dot{e}$ for each row by $t_{i}^{*}$. Therefore, the new allocation matrix will be arrived at as shown in Figure 3 .

Now, to check for optimality we form the vector

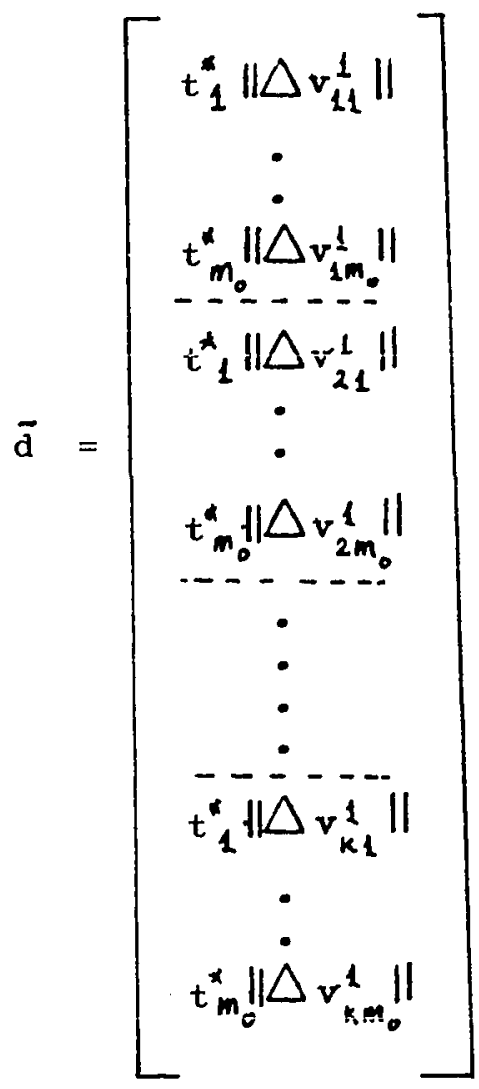




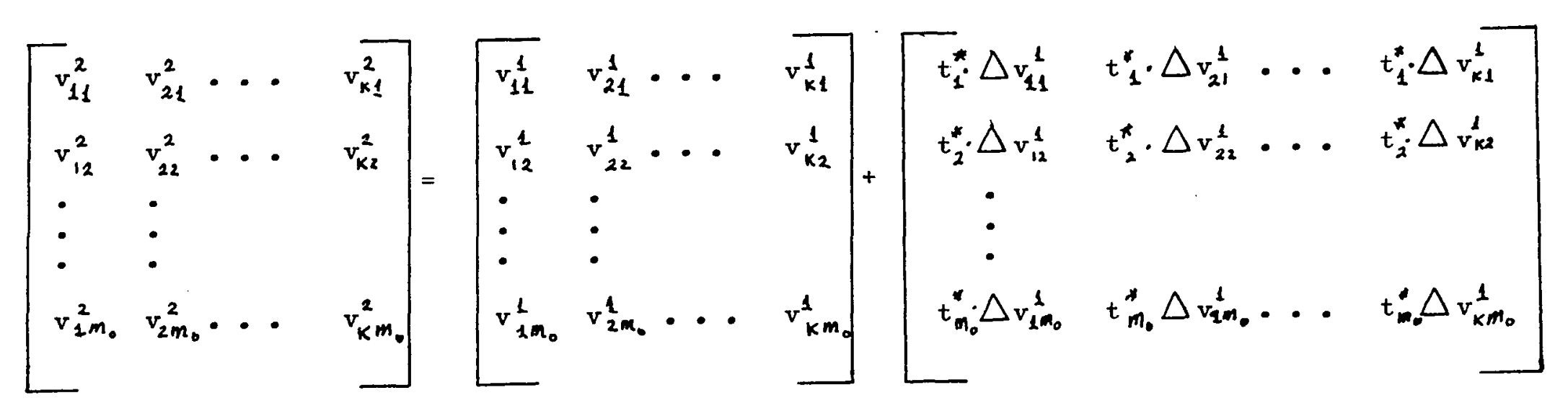

Figure 3

Calculation of resource reallocation 
and then we normalize it by

$$
\|\overline{\mathrm{d}}\|=\mathbf{1}
$$

Then by relation $(3.100)$ if

$$
\bar{w}^{\prime} \bar{d} \leqslant 0
$$

we have reached optimality.

\section{SUMMARY OF THE PROCEDURE}

Step 1. Allocate to each subsystem $e \overrightarrow{\mathrm{v}}_{\mathrm{e}}^{r}$ initial amounts of resources resulting from the arbitrary partition of the vector of central resources such that

$$
\sum_{l=1}^{l=k} \bar{v}_{l}^{r} \leqslant \bar{b}_{0}
$$

Step 2. Obtain the optimal dual variables $\vec{w}_{e}^{r}$ for $e=1, \ldots k$ by solving the subsystems.

Step 3. Obtain the mean optimal dual variable for each single resiource by the formula

$$
\text { mean } i=\frac{\sum_{\ell=I}^{\ell=k} w_{l i}\left(\bar{v}_{l}^{r}\right)}{k} \text { for } i=1, \ldots, \mathrm{m}_{0}
$$


Step 4. Obtain the changes for each optimal dual variable for each single resource by the formula

$$
\Delta w_{e i}\left(\bar{v}_{e}^{r}\right)=\text { mean } \mathbf{i}-w_{c i}\left(\bar{v}_{e}^{r}\right)
$$

for $i=1, \ldots, \mathrm{m}_{n}$, and $\ell=1, \ldots, \mathrm{k}$

Step 5. If $\bar{w}_{e}\left(\bar{v}_{e}^{r}\right) \neq \overline{0}$ go to Step 6 , otherwise check mean $i=0$, for all $i$ - If this holds, optimality has been reached and thus go to Step 13. Otherwise go to Step 7.

Step 6. Since $\bar{w}_{e}\left(\bar{v}_{e}^{r}\right)$ is unrestricted for $\ell=1, \ldots, k$ check if $\bar{w}_{\ell}\left(\bar{v}_{\ell}^{r}\right)=\overline{0}$ for $\ell=1, \ldots k$. If this holds optimality has been reached and thus go to Step 13. Otherwise go to Step 7.

Step 7. If $\Delta \bar{w}_{e}\left(\bar{v}_{e}^{r}\right)=\overline{0}$ for all $\ell$ then optimality has been reached and thus go to Step 13. Otherwise go to Step 8.

Step 8. Calculate the changes for each resource partition by the formula

$$
\Delta v_{e i}^{r}=-\left\{\frac{\Delta_{w_{e i}}\left(v_{e}^{r}\right)}{\sum_{l=1}^{l a k} w_{e i}\left(\bar{v}_{e}^{r}\right)}\right\}\left(\frac{b_{o i}}{k}\right)
$$

for $\ell=1, \ldots, k, \underset{k}{e+1} i=1, \ldots, m_{0}$

Step 9. Normalize the changes for the same resource

$$
\left\|\Delta v_{l i}^{r}\right\|=\frac{\Delta v_{e i}^{r}}{\sqrt{\left(\Delta v_{1 i}^{r}\right)^{2}+\left(\Delta v_{2 i}^{r}\right)^{2}+\ldots+\left(\Delta v_{k i}^{r}\right)^{2}}}
$$


Step 10. Calculate the maximum step $t_{i}^{*}$ by considering only the negative changes

$$
t_{i}^{*}=\max t_{i}=\min _{i}\left(\frac{v_{\ell i}^{1}}{\left|\Delta v_{\ell i}^{1}\right|}\right), \quad \ell=1, \ldots, \mathrm{k}
$$

for all $i$ for which $\triangle v_{e i}^{1}$ is negative.

Step 11. Find the new a 11ocations

$$
v_{e i}^{r+1}=v_{e i}^{r}+t_{i}^{*} \Delta v_{e i}^{r}
$$

for all $i$ and $\ell$, and go to step 12 .

Step 12. Form the vector $\bar{d}$ as shown in (4.26) and normalize it

$$
\|\bar{d}\|=1
$$

If $\quad \overline{\mathrm{w}}^{\prime} \overline{\mathrm{d}} \leqslant 0$

then optimality has been reached and therefore go to step 13. Otherwise set $r=r+1$ and go to step 2 .

Step 13. The optimum partition of $\bar{b}_{0}$ has been found and iherefore the optimum for the whole system

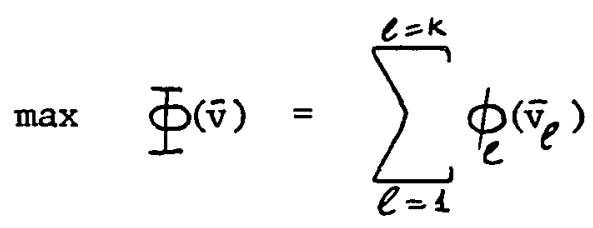

has been achieved, and therefore stop.

The procedure has been programmed and the experimental program and a numerical example are given in the Appendices. 
CHAPTER V

PRICE-RESOURCE DIRECTIVE COORDINATION: APPLICATIONS

The Price-Resource Directive Coordination procedure will be used here for the optimization of large-scale systems of the economic and technological type. The examples selected are from the economic planning, and industrial engineering fields and are such as to give good illustrations of the broad applicability of the procedure for decentralized optimal planning or control in complex systems. Also, the examples will give economic and technological content to the various steps of the procedure.

\section{DECENTRALIZED OPTIMAL ECONOMIC PLANNING}

Consider the general system $\mathrm{S}$, given in Figure 4, consisting of an arbitrary but finite number of interacting subsystems $\mathrm{s}_{\ell}(\ell=1$, $\ldots, k)$. For each subsystem $\ell$ we can define a set of inputs $I(\ell)$ arriving from the other subsystems and a set of outputs $0(\ell)$ going to the other subsystems.

With each branch leading from subsystem $\ell$ to subsystem $j$ we can associate in general a vector $\bar{a}_{e j}$ which can be called the production of subsystem $s_{\mathcal{l}}$ delivered to the subsystem $s_{j}$. Then the vector 


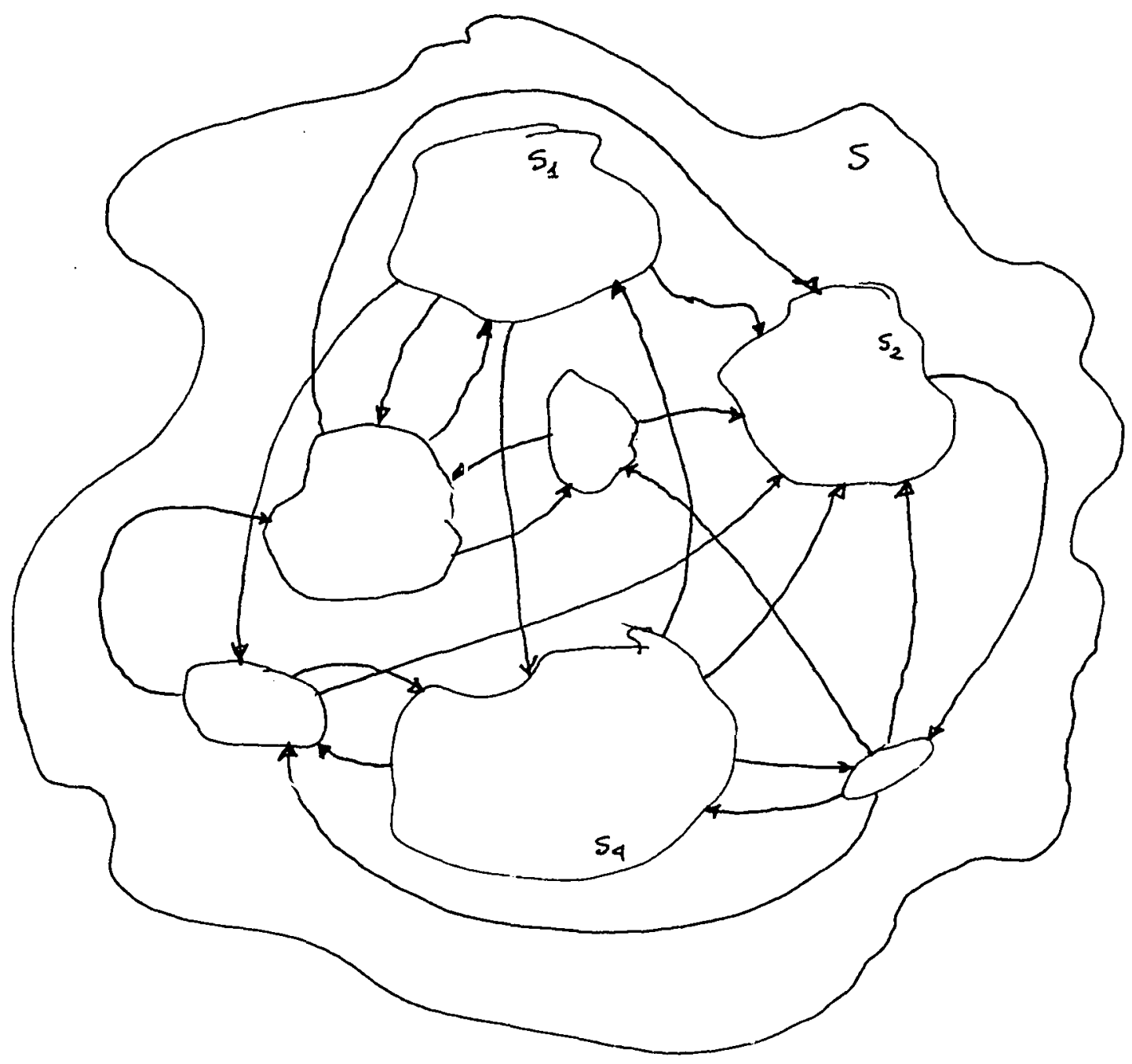

Figure 4. Genera1 system with interacting elements. 


$$
\bar{a}_{e}=\sum_{j \in O(\rho)} \bar{a}_{e j}
$$

is the total production of subsystem $\ell$.

If we assume that the production of the subsystems is delivered from subsystem to subsystem by stages and the process is finalized at a subsystem $k+1$, then the final production of the system will be

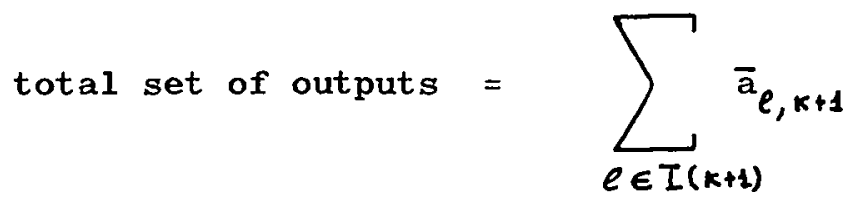

Also, we assume that the value $\bar{a}_{\ell}$ is dependent linearly on the intensity of utilization of the means of production vector $\bar{x}_{e}$, in other words

$$
\mathrm{B}_{e} \overline{\mathrm{x}}_{e}=\overline{\mathrm{a}}_{\ell} \quad(e=1, \ldots, \mathrm{k})
$$

where $\mathrm{B}_{C}$ is the output matrix.

The intensity of utilization of the means of production $\bar{x}_{e}$ of each subsystem $\ell$ depends on the delivery of production by the other subsystems of the system with which subsystem $\ell$ is interacting. Also, $\vec{x}_{e}$ depends on the resources to be found within the subsystem $C$. The dependence of $\bar{x}_{l}$ on external deliveries can be formulated mathematically as a constraint of mutual deliveries

$$
c_{e} \bar{x}_{e}=\sum_{j \in I(e)} G_{e j} \bar{a}_{j e} \quad(e=1, \ldots, k)
$$


where $C_{\mathcal{E}}$ is the cost matrix and $G_{e j}$ is the matrix of utilization of the deliveries.

The dependence of $\bar{x}_{l}$ on the eth subsystem resources can be given as

$$
\mathrm{D}_{e} \overline{\mathrm{x}}_{e}=\mathrm{b}_{e} \quad(e=1, \ldots, \mathrm{k})
$$

We also assume that the set of inputs to the whole system is given by

$$
\sum_{l=1}^{l-x} \bar{a}_{0, l}=\bar{b}_{0}
$$

where the index 0 indicates external deliveries.

Now, if the objective of the system $s$ is to maximize the value of the final production for constant prices $\mathrm{p}_{\ell}$, and the subsystems are relatively independent, in the sense that for coordination to achieve the objective, no direct information is needed about the local resources and structure of each subsystem by the system $s$, the optimization problem for the whole system $s$ can be divided into a number of extremal problems for each of the subsystems.

Let the objective function of $s$ be

$$
\max z=\sum_{l \in I(k+1)} \overline{\mathrm{p}}_{e}^{\prime} \overline{\mathrm{a}}_{e, k+1}
$$


then by relation $(5.3)$ and $(5.1)-(5.6)$ we have

$$
\max \quad \mathrm{z}=\sum_{\ell=1}^{\ell=k} \overline{\mathrm{p}}_{e}^{\prime} \mathrm{B}_{e} \overline{\mathrm{x}}_{e}
$$

subject to

$$
\begin{aligned}
\sum_{e=1}^{c=k} \bar{a}_{0, e} & =\bar{b}_{0} \\
c_{e} \bar{x}_{e} & =\sum_{j \in I(e)} G_{e, l} \bar{a}_{e, j} \\
D_{e} \bar{x}_{e} & =\bar{b}_{e} \\
\bar{x}_{e} & \geqslant \overline{0}
\end{aligned}
$$

Now, if the deliveries $\bar{a}_{0, e}$ are fixed at some level $\bar{a}_{0, e}=\bar{v}_{e} \leqslant \vec{b}_{o}$, the problem (5.8) - (5-11) decomposes into $k$ independent subproblems, whose solutions require only information available at the locel subsystem level. Therefore the subsystems have to solve their own problems according to the level of the deliveries according to

$$
\left.\phi_{l}\left(v_{l}\right)=\max \left\{\bar{p}_{l}^{\prime} B_{e} \bar{x}_{l}\right) c_{l} \bar{x}_{e}=G_{0, e} \bar{a}_{e, e}, D_{e} \bar{x}_{e}=\bar{b}_{e}, \bar{a}_{0, e}=\bar{v}_{e}\right\}
$$

From the preceding chapters we know that the achievement of the overall optimum for $s$ can be accomplished by the Price-Resource Directive Coordination procedure. In this economic system the "authorities" of the subsystems have several choices for establishing this type of coordination. One of them could be that they can select representatives 
from each subsystem and create a "coordination committee." This coordination committee will be responsible for the optimum allocation of the common resources of the whole system $s$. The only information that the committee will need in every stage of the planning process from the subsystems reduces to messages on the values of production $\phi_{l}\left(\vec{v}_{l}\right)$, corresponding to the different amounts of resources $\bar{v}_{l}$, and messages about the prices that each subsystem is willing to pay to acquire more of the common resources (the shadow prices).

The job of the committee is very easy, since according to the PriceResource Directive Coordination procedure, the only thing they have to do is agree on what consitutes $a$ fair average price and then find out the deviation of the prices offered by the subsystems. If a price is higher than the average they shift proportionally more common resources to that subsystem and reduce in the same proportion the common resources allocated to subsystems which gave prices less than the average. The information on the new allocations is transmitted to the subsystems which forces them to come up with new prices for the common resources more to the liking of the "coordination committee."

In this manner, in a finite number of coordination committee decisions, the optimal allocation of common resources is achieved. And, of course, this happens when the subsystems transmit to the committee identical prices. Then, there is no preference and the committee disolves itself. 


\section{MULTIFURNACE ALUMINUM ALLOY BLENDING}

This example explores in brief the use that can be made of the Price-Resource Coordination procedure in the aluminum alloying industry. It considers the problem of satisfying the requirements of an alloy or different alloys' specification most economically for consecutive charges of one furnace, or charges for several furnaces to be charged simultaneously.

The economical blending of an alloy is a complex process, but linear programming provides a means of examining all existing possible combinations and quickly arriving at the most economical furnace charge.

A single-furnace process encompasses one alloy and one furnace. Multifurnace processes may represent an entire alloying shop and several different alloy types.

The sources of the various alloy elements are the available raw materials and constitute the problem activities. The required minimization of the cost of blending will provide values for the intensity of utilization of the available raw materials. In our example, the activity intensities will be denoted by the vector

$$
\overline{\mathrm{x}}_{\ell}, \ell=1, \ldots, \mathrm{k}
$$

where $\ell$ stands for the appropriate furnace.

Each single-furnace operates under certain constraints. These are: (a) element specifications (E.S.) which constraint the amount of 
each ingredient metal that may be charged. The coefficients in each row of these constraints are the amounts of the element (represented by the row) in one pound of each activity (raw material), and (b) raw Materials availability constraints (R.M.A.j which are constraints on the maximum or minimum permissiblc level of utjilization of the raw materials.

The whole shop or factory which has $k$ furraces is constrained by the raw materials' availability and constraints which specify the amounts of different alloys to be blended.

The objective of the whole operation being the minimization of the cost of blending, the total system objective function consists of the per-pound costs of each raw material multiplied by the intensity of uti1ization of that raw material.

In mathematical programming modeling terms the example can be given as follows:

Minimize total system $\cos t \quad \bar{c} \bar{x}$ subject to

\begin{tabular}{|c|c|c|c|}
\hline \multicolumn{3}{|c|}{ CENTRAT CONSTRAINTS } & $\begin{array}{l}\text { Furnace Loads } \\
\text { Inventory } \\
\text { Availability }\end{array}$ \\
\hline \multirow[t]{3}{*}{$\begin{array}{l}\text { A1loy } x \\
\text { Furnace } 1\end{array}$} & & \multirow[b]{3}{*}{ • } & $\begin{array}{l}\text { A11oy } x \\
\text { (E.S.) } \\
\text { (R.M.A.) }\end{array}$ \\
\hline & $\begin{array}{c}\text { Alloy } y \\
\text { Furnace } 2\end{array}$ & & $\begin{array}{l}\text { A11oy y } \\
\text { (E.S.) } \\
\text { (R.M.A.) }\end{array}$ \\
\hline & & & $\dot{\bullet}:$ \\
\hline
\end{tabular}


As it can be seen, the multifurnace aluminum blending problem has a block-angular structure and therefore it can be solved by the PriceResource Directive Coordination procedure. As described in the previous chapters, this system can be decomposed by an initial allocation, by the management of the enterprise, of the available raw materials to the different furnaces. Once this is done, each furnace foreman in his own computer time-sharing terminal calculates the cost of producing his assigned alloy for the initial raw materials allocated to him, and the variation in cost for a small change in this allocation. These two figures are sent to the management of the enterprise by each furnace foreman. The management calculates the average cost variation for the whole enterprise and the difference from this average for each furnace, then, according to this difference, proportiona11y allocates more raw materials to the furnaces that are below the average and less to those that are above the average. These new allocations are sent back to the furnace foremen for evaluation. This iterative process terminates when the management is satisfied that each furnace will change the cost in the same proportion given any change in allocation.

The Price-Resource Directive Coordination procedure here, apart from solving the total enterprise optimization problem, also provides the management with a detailed picture of costing for each subsystem furnace which can reveal hidden equipment obsolescence or personnel overstaffing. 
1. Hollander, Gerhard L., "Synthesis of Major Systems: An Open Challenge," 0. R./S. A. Today, Vo1. w, No. 2 (Apri1, 1972).

2. Johnson, R. A., F. E. Kast, and I. E. Rosenzweig, The Theory of Management of Systems, McGraw-Hil1 Book Co., New York, 1963.

3. Fleyshman, B. S., "Results and Problems in Efficiency of Complex Systems," Engineering Cybernetics, No. 4 (1970).

4. Lasdon, Leon S., "Duality and Decomposition in Mathematical Programming," IEEE Transactions on Systems Science and Cybernetics, Vol. SSC-4, No. 2 (July, 1968).

5. Pearson, J. D., "Decomposition, Coordination, and Multilevel Systems," IEEE Transactions on Systems Science and Cybernetics, Vo1. SSC-2, No. 1 (August, 1966).

6. Tomasini, Luigi M., "Toward a General Economic System Theory," Metroeconomica, Vo1. 23 (Apri1, 1971).

7. Chukhov, A. I., "Certain Questions on Optimization of a Contro1 System for a Large Project with a Hierarchical Structure," Ekonomika i. Matematicheskie Metody, No. 3 (Fa11, 1968).

8. Mesarovic, M. D., "Conceptual Framework for the Study of Large Systems," Systems Research Center Report SRC 77-A-65-29, Case Western Reserve University, Cleveland, Ohio, 1965.

9. Smithies, Arthur, "PPBS, Suboptimization and Decentralization," Rand Memorandum RM-6178-PR, The Rand Corporation, Santa Monica, California (Apri1, 1970).

10. Beer, Stafford, Management Science, Doubleday \& Co., Inc., Garden City, New York, 1968.

11. Yudin, D. B., "Decision-Making in Complex Situations," Engineering Cybernetics, No. 2 (1970).

12. Linstone, Harold A., "Four American Futures: Reflections on the Role of Planning," Systems Science Report 72-1, Systems Science Institute, Portland State University, Portland, Oregon (February, 1972). 
13. Mesarovic, M. D., D. Macko and Y. Takahara, Theory of Hierarchical Multileve1 Systems, Academic Press, New York, 1970.

14. Sayles, Leonard R., and Margaret K. Chandler, Managing Large Systems, Organizations for the Future, Harper \& Row Publishers, New York, 1971.

15. Drenick, Rudolf, "Review of Theory of Hierarchical Multilevel Systems Authored by Mesarovic et. a1.," Operations Research, Vo1. 19, No. 1. (January-February, 1971).

16. Bus lenko, N. P., "The Theory of Complex Systems," Engineering Cybernetics, No. 5 (1963).

17. Yudin, D. B., "Methods of Quantitative Analys is of Complex Systems I," Engineering Cybernetics, No. 1 (1965).

18. Yudin, D. B., "Methods of Quantitative Analysis of Complex Systems II," Engineering Cybernetics, No. 1 (1966).

19. Gomory, R., "Large and Non-Convex Problems in Linear Programming," Proceedings of Symposia in Applied Mathematics, Vo1. XV, American Mathematical Society (1963).

20. Dantzig, G. B., and A. Madansky, "On the Solution of Two-Stage Linear Programs Under Uncertainty," in Proceedings of the Fourth Symposium on Mathematical Statistics and Probability, Vo1. I, University of California Press, Berkeley (1961), pp. 165-176.

21. Van Slyke and Wets, R. J. B., "L-Shaped Linear Programs with Applications to Optimal Control and Stochastic Programming," SIAM Journal of Applied Mathematics, Vol. 17, No. 4 (July, 1969), pp. 638-663.

22. Selser, Gregorio, Espionaje en America Latina: el Pentagono y las Tecnicas Sociologicas, Impreso en Mexico, 1967.

23. Rijnsdorp, J. E., "Multilevel Systems in the Petroleum Industry," Automatica, Vol. 8 (1972), pp. 105-106.

24. Klir, J., and M. Valach, Gyberne iic Modelling, Illiffe Books, London, 1967.

25. Aronovich, V. V., G. P. Gavrilov and R. F. Romm, "Hierarchical Algorithms for Optimal Control of Chemica1-Technological Complexes," Artomatika i Telemekhanika (Automation and Remote Contro1), No. 10 (October, 1970), pp. 133-145. 
26. Dantzig, George B., "Large Scale Linear Programming," in Mathematics of the Decision Sciences, Part 1 (ed.) G. B. Dantzig and A. F. Veinott, American Mathematical Society, Providence, Rhode Is land (1968), pp. 77-92.

27. Fox, Richard L., Optimization Methods for Engineering Design, Addison-Hesley Publishing Company, Reading, Massachusetts, 1971.

28. Qayum, Abdul, Methods of Economic Planning, to be published by Indiana University Press.

29. Wagner, Harvey, Principles of Operations Research, with Applications to Managerial Decisions, Prentice-Ha11, Inc., Englewood Cliffs, New Jersey, 1969.

30. Hyvarinen, Lassi, Mathematical Modeling for Industrial Processes, Springer-Ver 1ag, New York, 1970.

31. Kulikowski, R., "Optimization of Large-Scale Systems," Automatica, Vo1. 6 (1970), pp. 315-331.

32. Wismer, D. A. (ed.), Optimization Methods for Large-Scale Systems, McGraw-Hil1 Book Company, New York, 1970.

33. Ven, V. I., and A. I. Erilike, "Some Questions in the Aggregation of Linear Mode1s," Engineering Cybernetics, No. 5 (1970), pp. 823-829.

34. Lasdon, Leon S., Optimization Theory for Large Systems, The MacMillan Company, New York, 1970.

35. Weil, R. L., and P. C. Kettler, "Rearranging Matrices to BlockAngular Form for Decomposition (and Other) Algorithms," Management Science, Vol. 18, No. 1 (September, 1971), PP. 98-108.

36. Geoffrion, Arthur M., "Elements of Large-Scale Mathematical Programming" in Perspectives on Optimization: A collection of Expository Articles (ed.) Arthur M. Geoffrion, AddisonWesley Publishing Company, Reading, Massachusetts (1972), pp. 25-64.

37. Simonnard, Miche1, Linear Programming, Prentice-Hal1, Inc., Englewood Cliffs, New Jersey, 1966 (translation from origina1 French, Dunod, Paris, 1962).

38. Dantzig, George B., Linear Programming and Extensions, Princeton University Press, Princeton, New Jersey, 1963. 
39. Hillier, Frederick S., and Gerald J. Lieberman, Introduction to Operations Research, Holden-Day, Inc., San Francisco, California, 1967.

40. Hu, T. C., Integer Programming and Network Flows, Addison-Wes ley Publishing Company, Reading Massachusetts, 1969.

41. Zoutendijk, G., Methods of Feasible Directions, E1sevier Publishing Company, New York, 1960.

42. John, F., "Extremum Problems with Inequalities as Subsidiary Conditions," in K. 0. Friedrichs, 0. E. Neugebauer, and J. J. Stoker (eds.), Studies and Essays: Curant Anniversary Volume, Pp. 187-204, Interscience Publishers, New York, 1948 .

43. Kuhn, H. W., and A. W. Tucker, "Non1inear Programming," in J. Neyman (ed.), Proceedings of the Second Berkeley Symposium on Mathematical Statistics and Probability, pp. 481-492, University of California Press, Berkeley, California, 1951.

44. Bernholtz, B., "A New Derivation of the Kuhn-Tucker Conditions," Operations Research, Vo1. 12 (1964), pp. 295-299.

45. Dorn, W. S., "On Lagrange Mu1tipliers and Inequalities," Operations Research, Vol. 9 (1961), pp. 95-104.

46. Falk, James E., "Lagrange Multipliers and Nonlinear Programming," Journal of Mathematical Analys is and Applications, Vo1. 19 (1967), pp. 141-159.

47. Danskin, John M., "The Theory of Max-Min, with Applications," J. SIAM on Applied Mathematics, Vo1. 14, No. 4 (July, 1966), PP. 641-664.

48. Bram, Joseph, "The Lagrange Multiplier Theorem for Max-Min with Several Constraints," J. SIAM on Applied Mathematics, Vo1. 14, No. 4 (July, 1966), pp. 665-667.

49. Wets, R. J. Br, "Programming Under Uncertainty: The Equivalent Convex Program," J. SIAM on Applied Mathematics, Vo1. 14, No. 1 (January, 1966), pp. 89-105.

50. Silverman, Gary J., "Primal Decomposition of Mathematical Programs by Resource Allocation: I - Basic Theory and a DirectionFinding Procedure," Operations Research, Vol. 20, No. 1 (January-February, 1972), pp. 58-74. 
51. Silverman, Gary J., "Primal Decomposition of Mathematical Programs by Resource Allocation: II - Computational Algorithm with an Application to the Modular Design Problem," Operations Research, Vo1. 20, No. 1 (January-February, 1972), pp. 75-93.

52. Geoffrion, Arthur M., "Prima1 Resource-Directive Approaches for Optimizing Nonlinear Decomposable Systems," Operations Research, Vol. 18, No. 3 (May-June, 1970), pp. 375-403.

53. Bazaraa, M. S., J. J. Goode, and C. M. Shetty, "Optimality Criteria in Nonlinear Programming Without Differentiability," Operations Research, Vo1. 19, No. 1 (January-February, 1971), pp. 77-86.

54. Peterson, Elmor L., "An Economic Interpretation of Duality in Linear Programming," Journal of Mathematical Analysis and Applications, Vo1. 30 (1970), pp. 172-196.

55. Kornai, J., "Multi-Level Programming - A First Report on the Mode1 and on the Experimental Computations," European Economic Review, Vo1. 1, No. 1 (Fa11, 1969), pp. 134-191.

56. Zschau, Edwin V. W., A Primal Decomposition Algorithm for Linear Programming, Working Paper 非91, Graduate School of Business, Stanford University, January, 1967.

57. Kornai, J., and Th. Liptak, "Two-Level Planning," Econometrica, Vo1. 33, No. 1 (January, 1965), pp. 141-169.

58. Zschau, Edwin V. W., A Theory of Primal Decomposition for Linear Programing, Working Paper 非120, Graduate Schoo1 of Business, Stanford University, February, 1967.

59. Rockafellar, Tyrrel1 R,, Convex Analysis, Princeton University Press, Princeton, New Jersey, 1970.

60. Ermol'er, M., "Methods of Solution of Nonlinear Extrema1 Problems," Cybernetics, Vol. 2, No. 4 (1966), pp. 1-17.

61. Van Slyke and R. Wets, "Programming under Uncertainty and Stochastic Optima1 Contro1," SIAM Journal on Contro1, Vo1. 4, No. 1 (February, 1966), pp. 179-193.

62. Geoffrion, Arthur M., "Large-Scale Linear and Nonlinear Programing," in (32), pp. 47-74.

63. Karlin, S., Mathematical Methods and Theory in Games, Programming, and Economics, Addison-Wesley Publishing Company, Reading, Massachusetts, 1959. 
64. Mangasarian, 01i L., Non1inear Programming, McGraw-Hil1 Book Company, New York, 1969.

65. Dantzig, G. B., and P. Wolfe, "The Decomposition Algorithm for Linear Programs," Econometrica, Vo1. 29, No. 4 (October, 1961), Pp. 767-778.

66. Dantzig, G. B., and P. Wolfe, "Decomposition Principle for Linear Programs," Operations Research, Vo1. 8, No. 1 (JanuaryFebruary, 1960), pp. 101-111.

67. Dantzig, G. B., "Mathematical Programming: Decomposition," Chapter 6, in Julius S. Aronofsky (ed.), Progress in Operations Research, pp. 177-193, John Wiley and Sons, New York, 1969.

68. Harvey, R. P., "The Decomposition Principle for Linear Programs," International Journal of Computer Mathematics (1964), pp. 20-35.

69. Baumo1, W. J., and T. Fabian, "Decomposition, Pricing for Decentralization and External Economies," Management Science, Vo1. 11, No. 1 (September, 1964), pp. 1-32.

70. Dantzig, G. B., "On the Status of Multistage Linear Programming Problems," Management Science, Vo1. 6 (October-July, 19591960), pp. 53-71.

71. Dantzig, G. B., "Recent Advances in Linear Programming," Management Science, Vol. 2, No. 2 (1956), pp. 131-144. 


\section{APPENDTX A}

\section{NUMERICAL EXAMPLES}

Consider the following block-angular structured linear programming problem.

$$
\left[\begin{array}{l}
x_{1} \\
x_{2} \\
x_{3} \\
x_{4} \\
x_{5} \\
x_{6} \\
x_{7}
\end{array}\right]
$$

A. 1 subject to

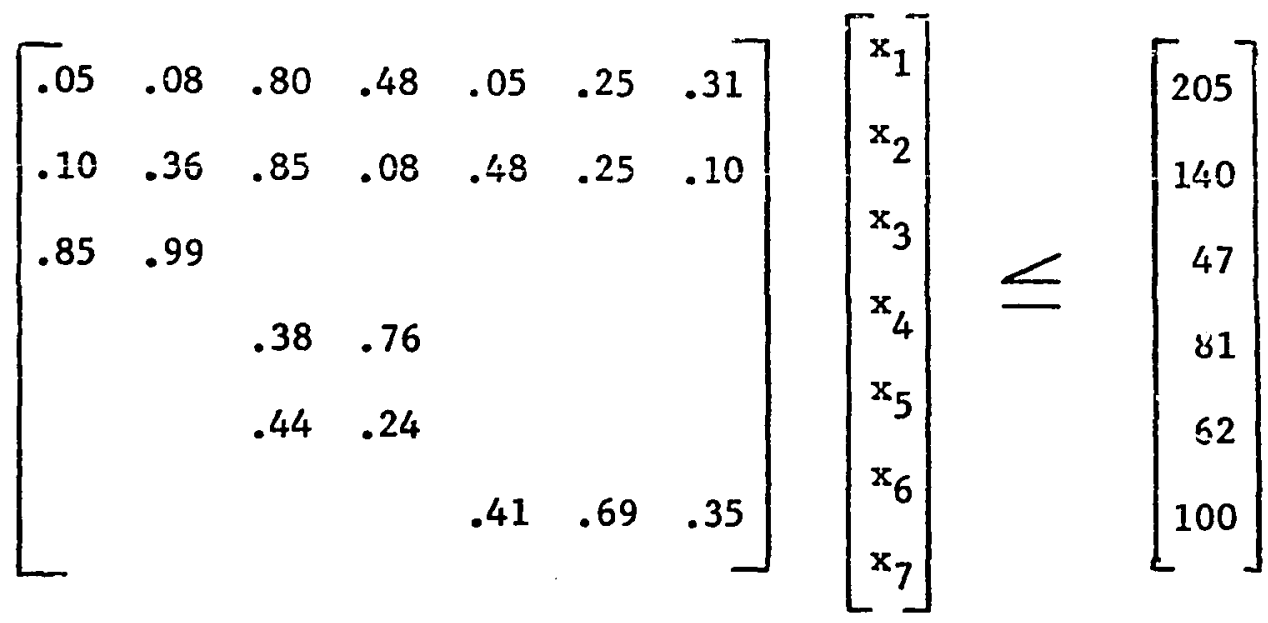

and a11 $x_{i} \geq 0, i=1, \ldots, 7$. 
The solution of A.1 utilizing a standard linear programming code without decomposition is as follows:

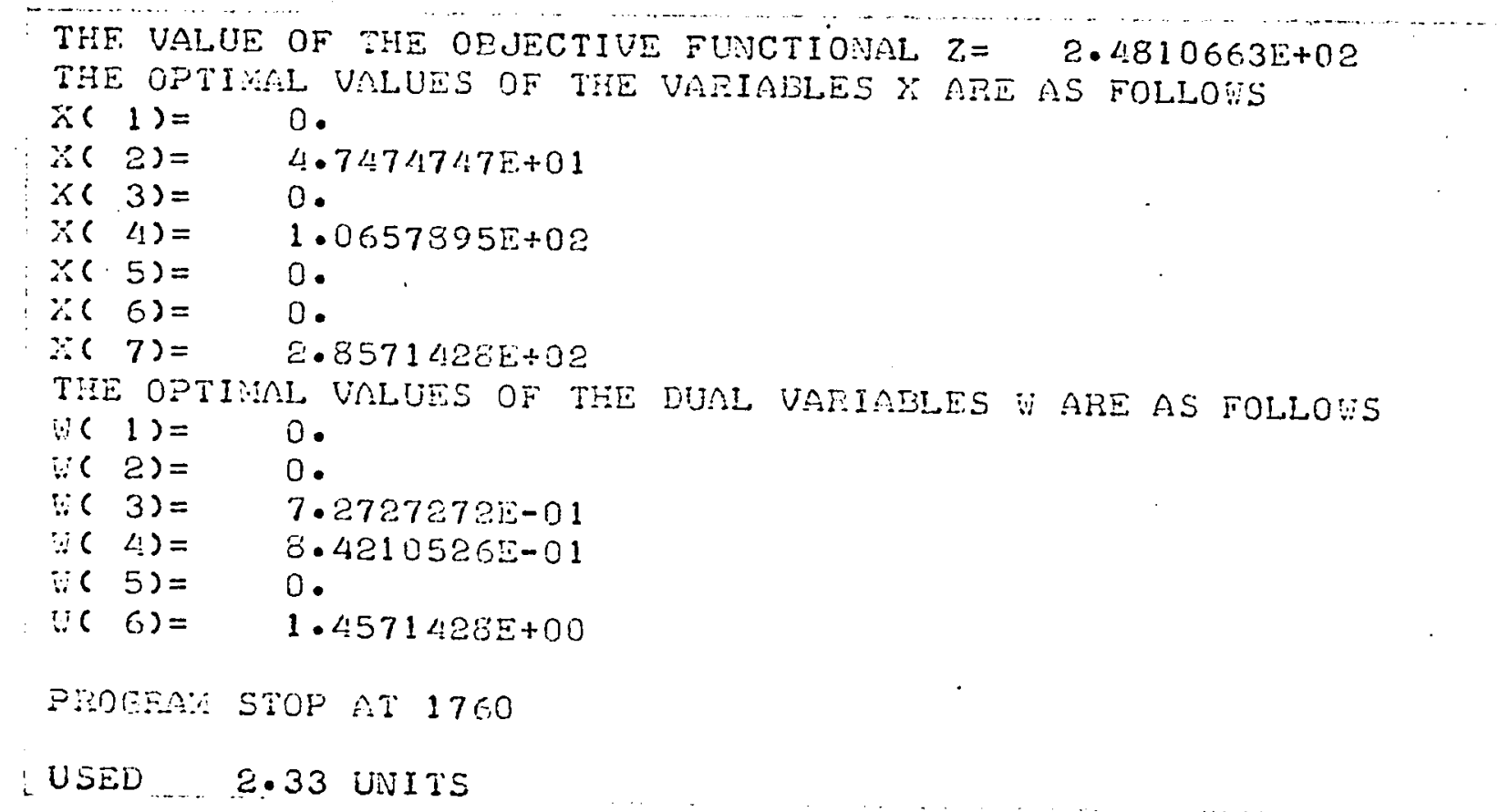

Now, we can refo:mulate A.I into a Central System and three Subsys tems.

Centraa yㅗ늠

$$
\begin{aligned}
& \text { Maximize } \Phi(\bar{v})=\phi_{1}\left(\bar{v}_{1}\right)+\phi_{2}\left(\bar{v}_{2}\right)+\phi_{3}\left(\bar{v}_{3}\right) \\
& \bar{v}_{1}, \bar{v}_{2}, \bar{v}_{3} \\
& \text { subject to } \\
& v_{11}+v_{21}+v_{31} \leq 205 \\
& v_{12}+v_{22}+v_{32} \leq 140
\end{aligned}
$$


where

Subsystem 1

$$
\phi_{1}\left(\bar{v}_{1}\right)=\max _{\bar{x}_{1}} \quad\left[\begin{array}{ll}
.40 & .72
\end{array}\right]\left[\begin{array}{l}
x_{11} \\
x_{12}
\end{array}\right]
$$

subject to

$$
\left[\begin{array}{ll}
.05 & .08 \\
.10 & .36 \\
.85 & .99
\end{array}\right]\left[\begin{array}{l}
x_{11} \\
x_{12}
\end{array}\right] \leq\left[\begin{array}{l}
v_{11} \\
v_{12} \\
47
\end{array}\right]
$$

Subsystem 2

$$
\phi_{2}\left(\bar{v}_{2}\right)=\max _{\bar{x}_{2}}\left[\begin{array}{ll}
.28 & .64
\end{array}\right]\left[\begin{array}{l}
x_{21} \\
x_{22}
\end{array}\right] .
$$

subject to

$$
\left[\begin{array}{cc}
.80 & .48 \\
.85 & .08 \\
.38 & .76 \\
.44 & .24
\end{array}\right]\left[\begin{array}{l}
x_{21} \\
x_{22}
\end{array}\right] \leq\left[\begin{array}{l}
v_{21} \\
v_{22} \\
81 \\
62
\end{array}\right]
$$

Subsystem 3

$$
\phi_{3}\left(\bar{v}_{3}\right)=\max _{\bar{x}_{3}}\left[\begin{array}{lll}
.32 & .60 & .51
\end{array}\right]\left[\begin{array}{l}
x_{31} \\
x_{32} \\
x_{33}
\end{array}\right]
$$

subject to

$$
\left[\begin{array}{lll}
.05 & .25 & .31 \\
.48 & .25 & .10 \\
.41 & .69 & .35
\end{array}\right]\left[\begin{array}{l}
x_{31} \\
x_{32} \\
x_{33}
\end{array}\right] \leq\left[\begin{array}{l}
v_{31} \\
v_{32} \\
100
\end{array}\right]
$$


We solve the decomposed system by the Price-Resource Directive Coordination procedure utilizing different initial feasible partitions of the Central System resource vector

$$
\vec{b}_{0}=\left[\begin{array}{l}
205 \\
140
\end{array}\right]
$$

Solutions

First Trial

Let the initial partition be

$$
\bar{v}_{1}=\left[\begin{array}{l}
40 \\
25
\end{array}\right] \quad \bar{v}_{2}=\left[\begin{array}{l}
110 \\
40
\end{array}\right] \quad \bar{v}_{3}=\left[\begin{array}{l}
55 \\
75
\end{array}\right]
$$

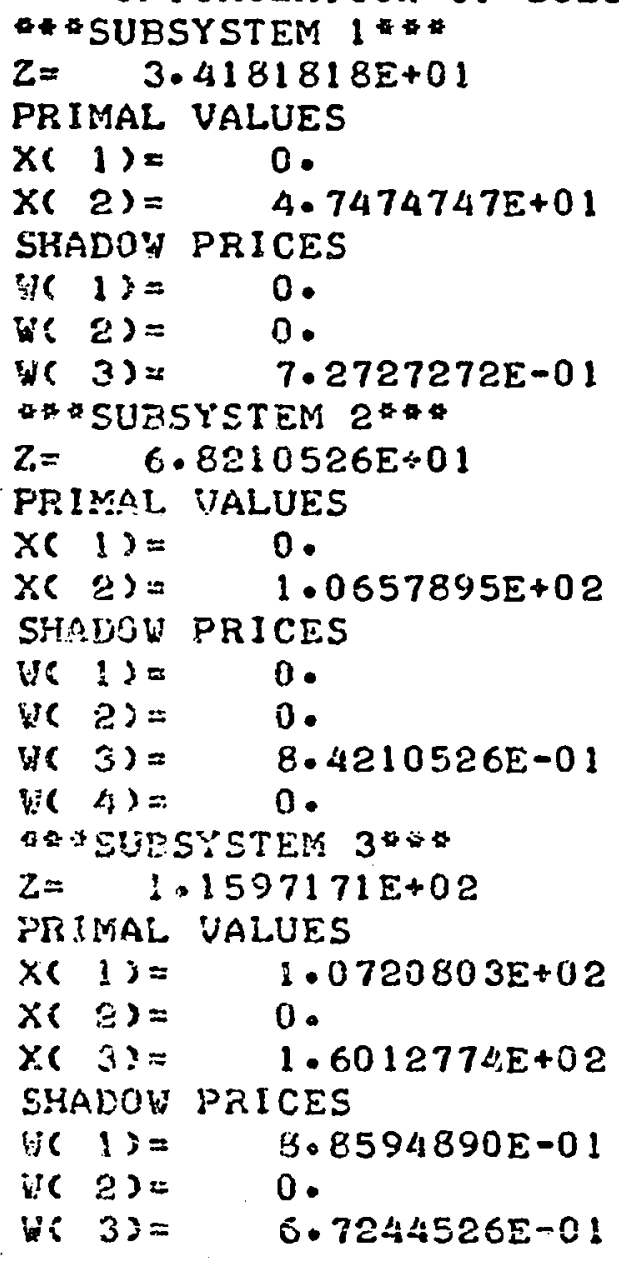




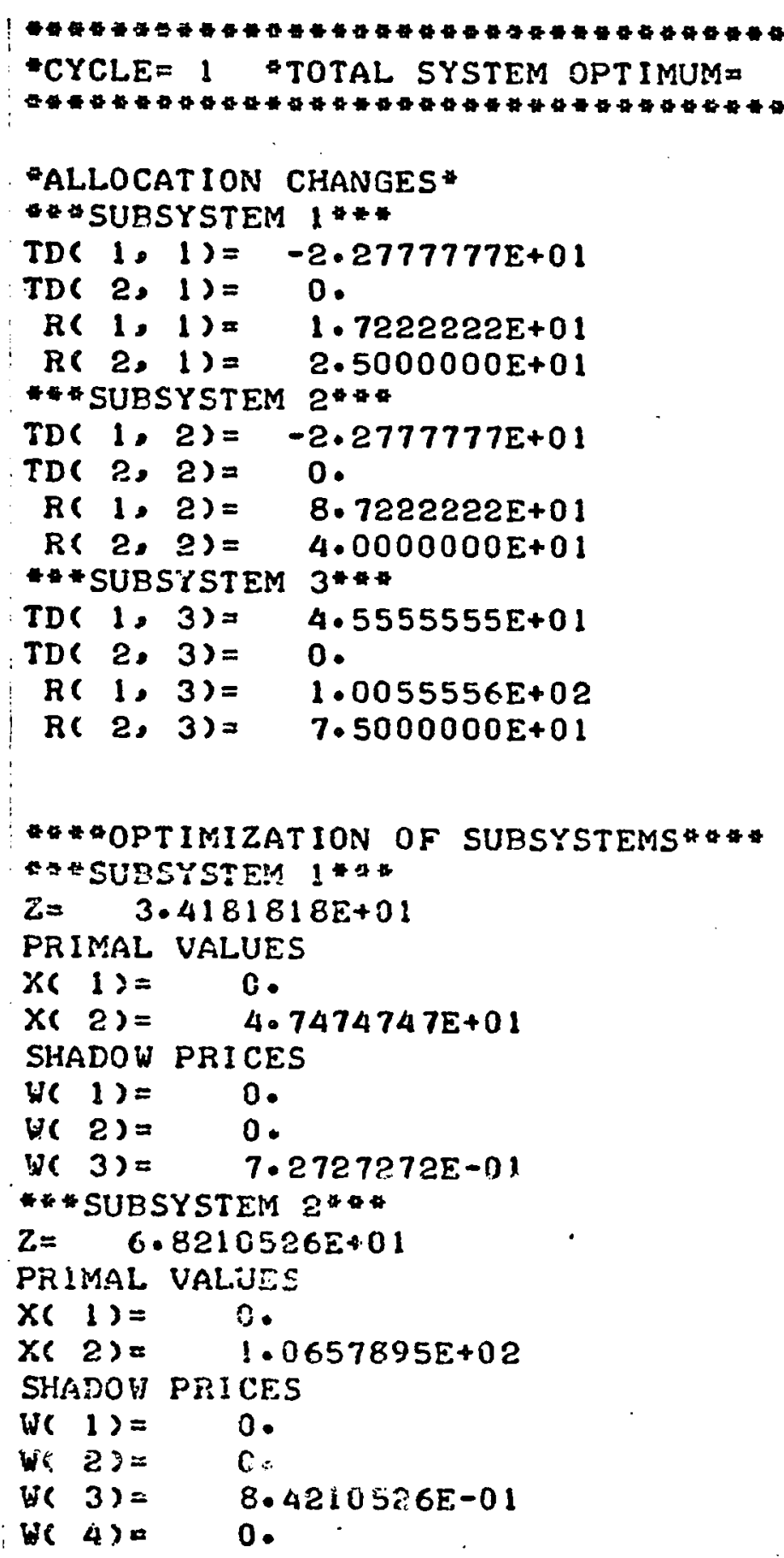




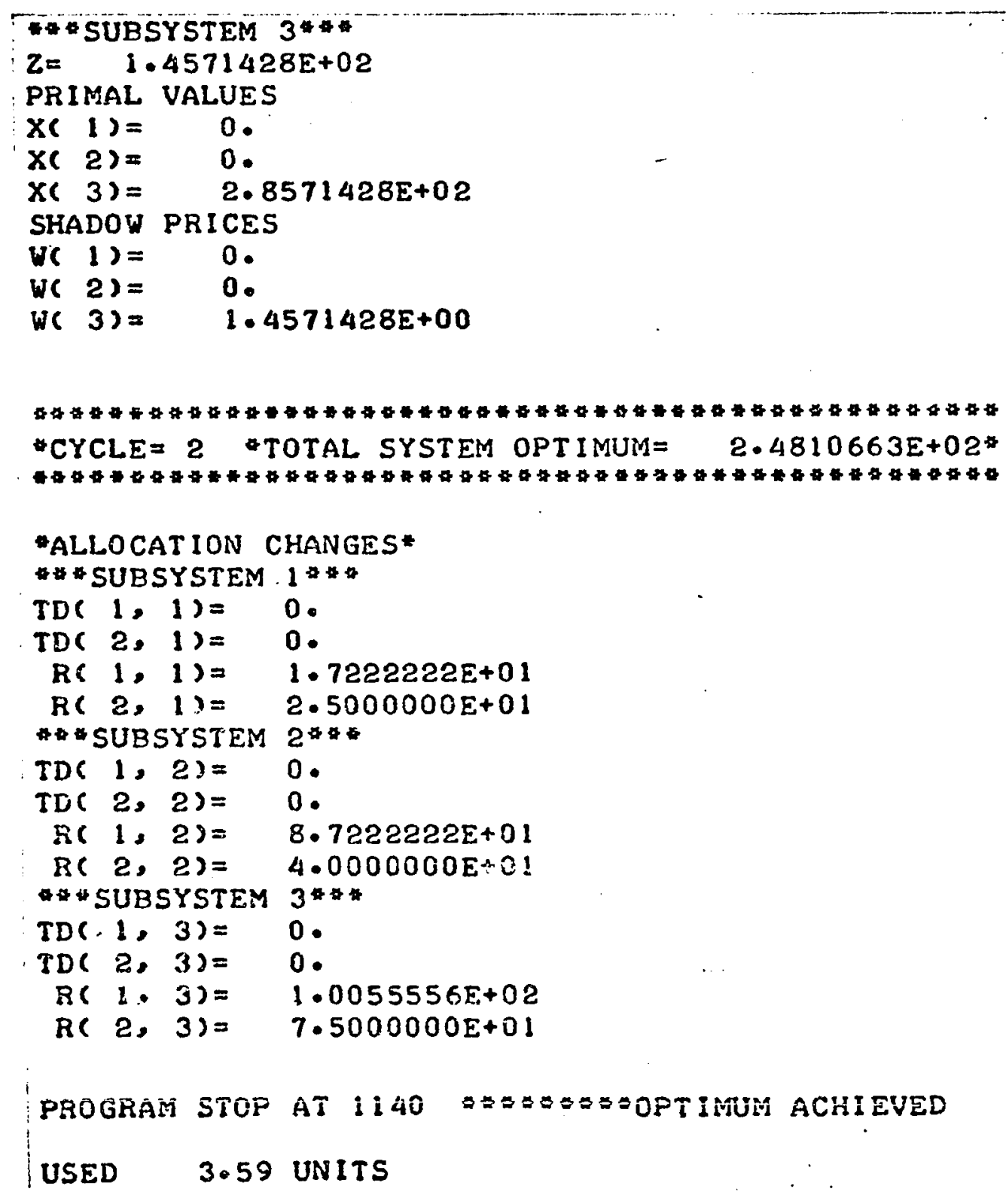


Second Trial

Let the initial partition be

$$
v_{1}=\left[\begin{array}{c}
5.0 \\
25.0
\end{array}\right] \quad v_{2}=\left[\begin{array}{l}
59.0 \\
40.0
\end{array}\right] \quad v_{3}=\left[\begin{array}{c}
141.0 \\
75.0
\end{array}\right]
$$

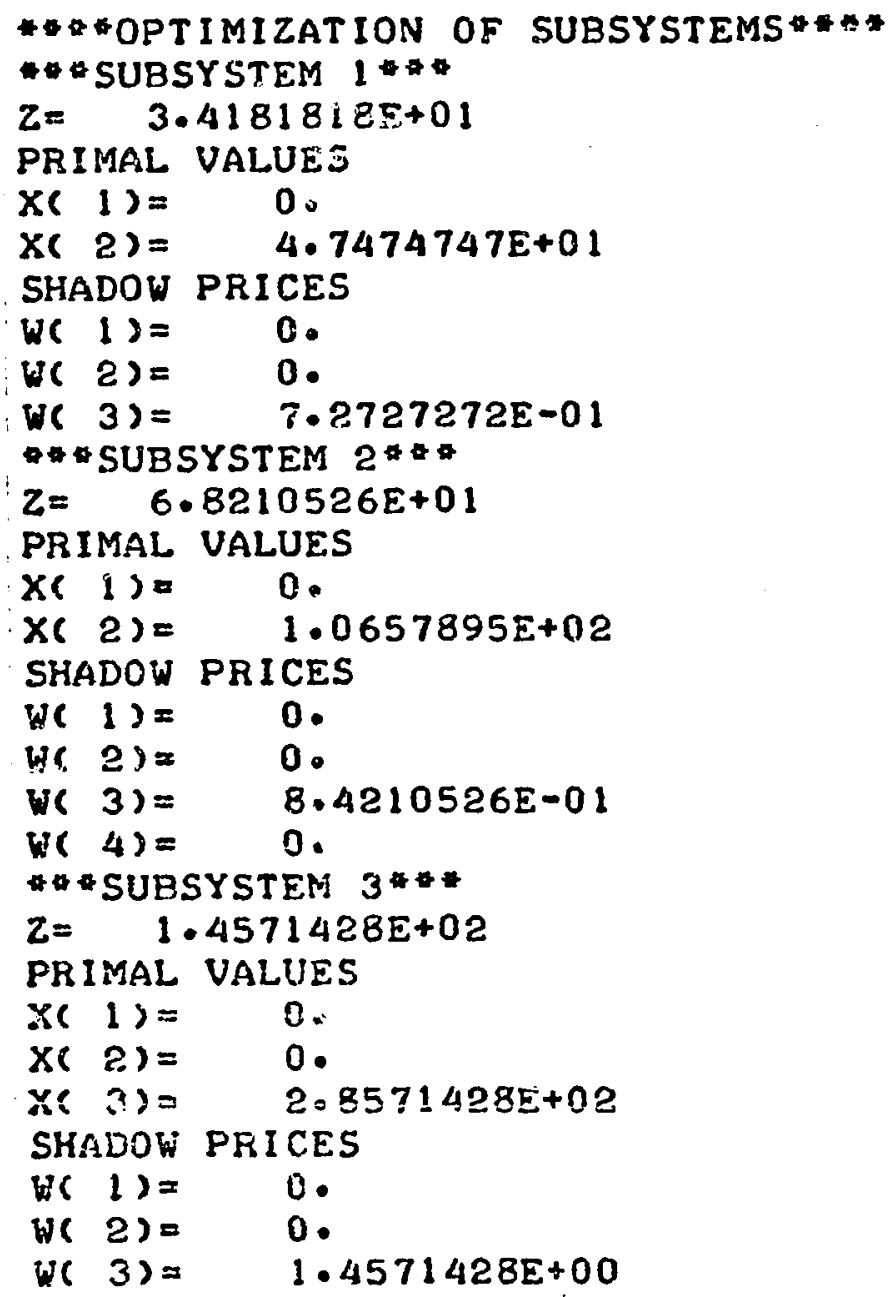




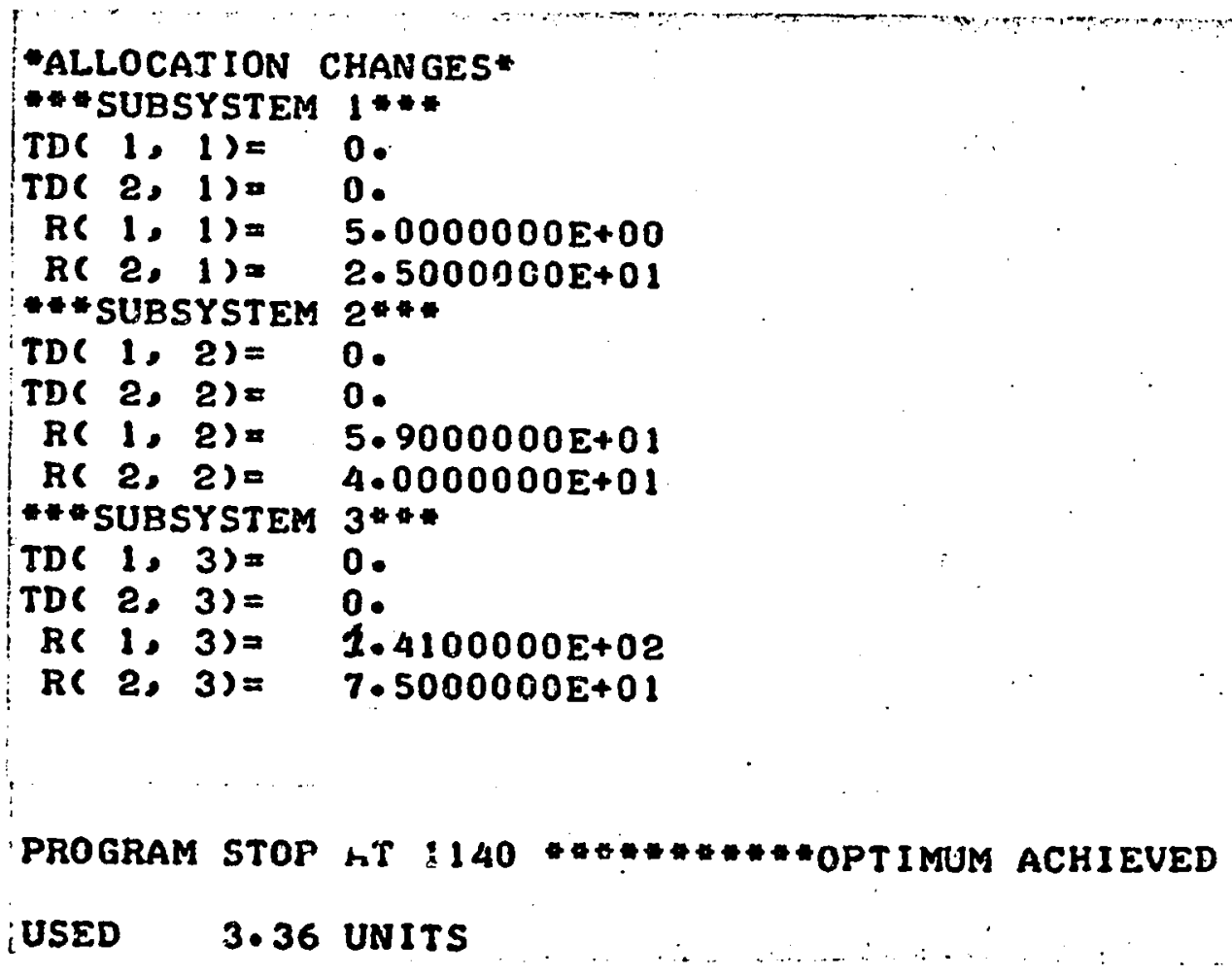

Third $\underline{\operatorname{Tr}} \underline{\underline{a}} \underline{\mathrm{1}}$

Let the initial partition be

$$
\bar{v}_{1}=\left[\begin{array}{l}
100.0 \\
42.0
\end{array}\right] \quad \bar{v}_{2}=\left[\begin{array}{l}
33.0 \\
81.0
\end{array}\right] \quad \bar{v}_{3}=\left[\begin{array}{l}
72.0 \\
17.0
\end{array}\right]
$$




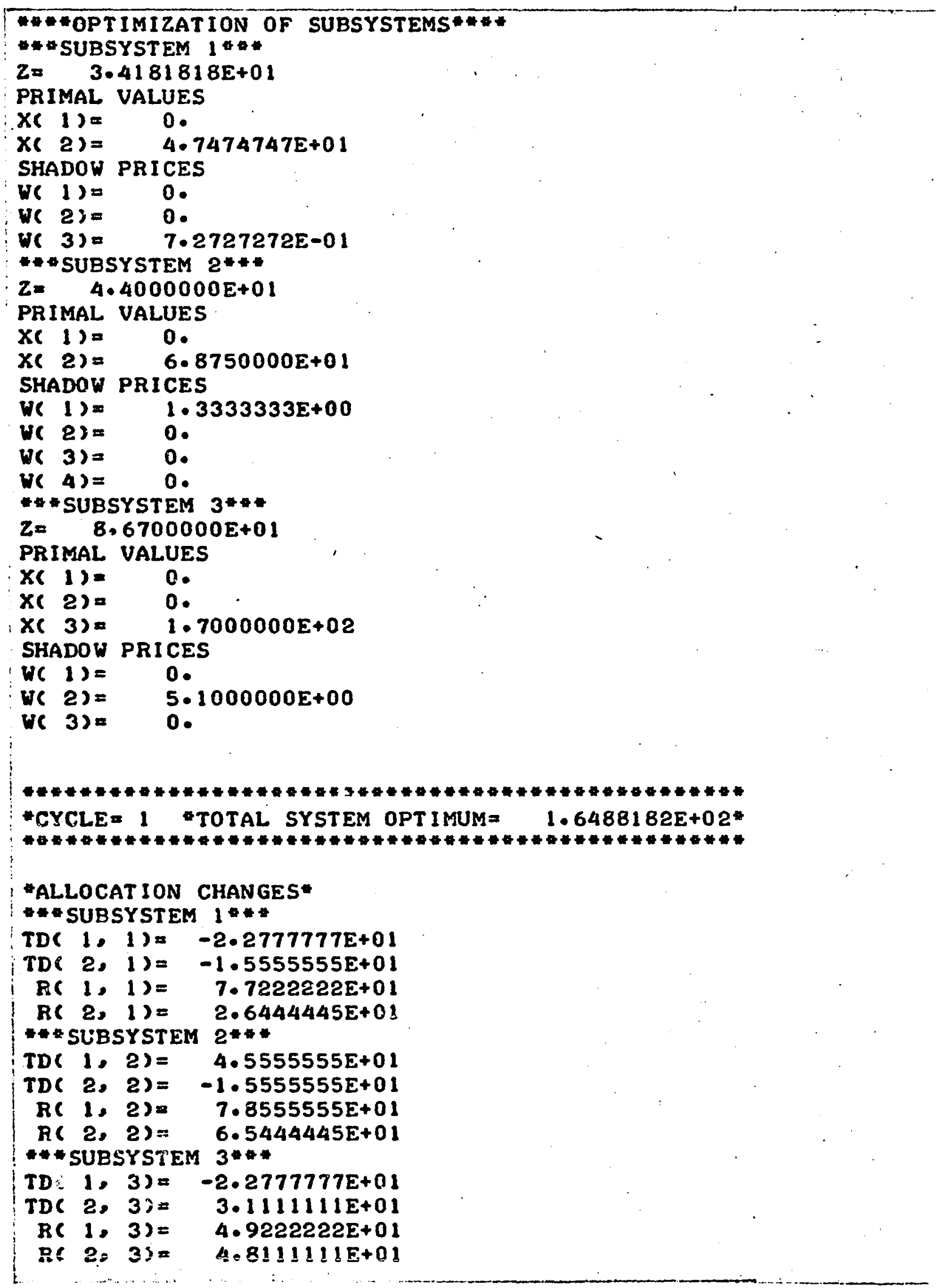




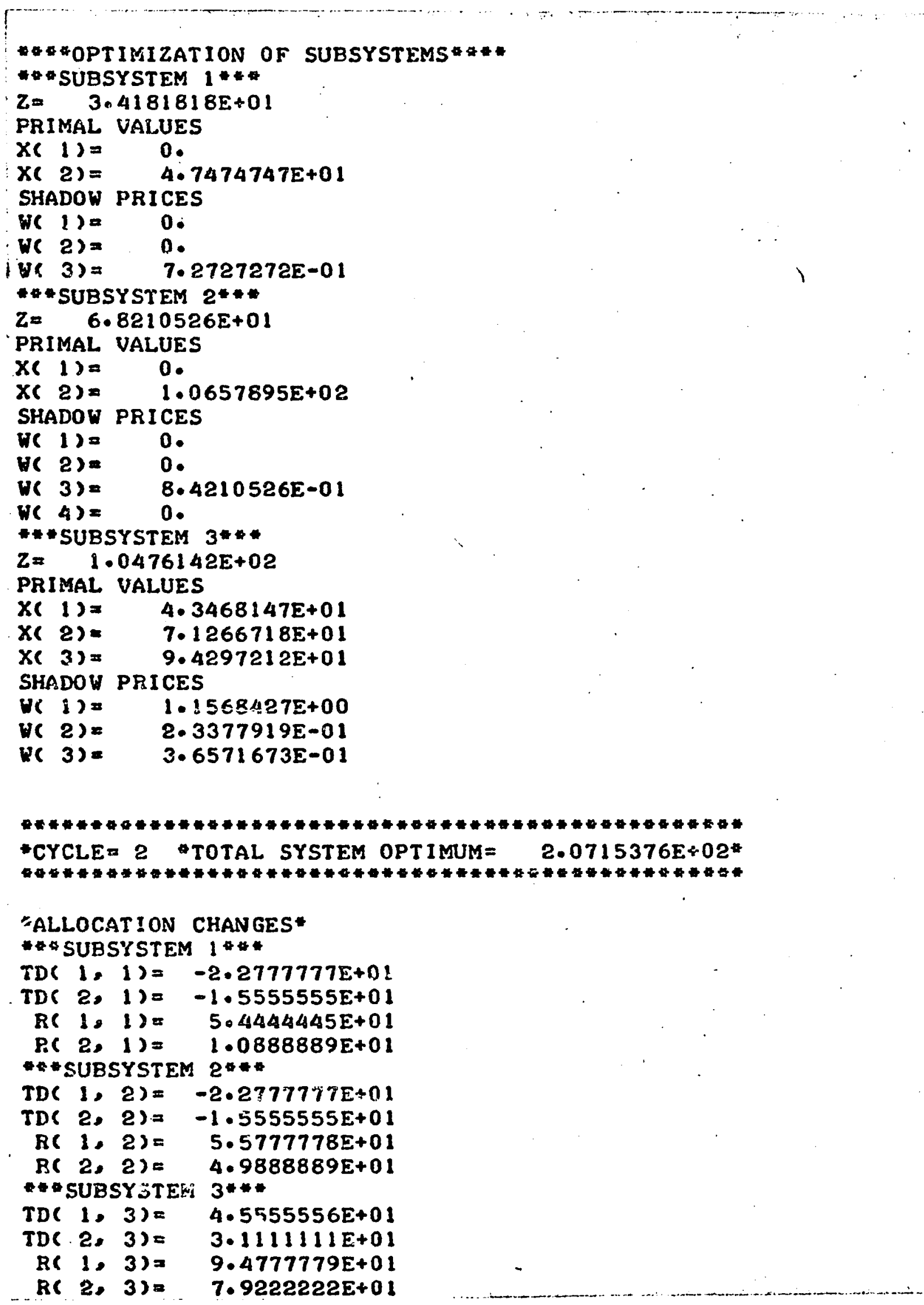




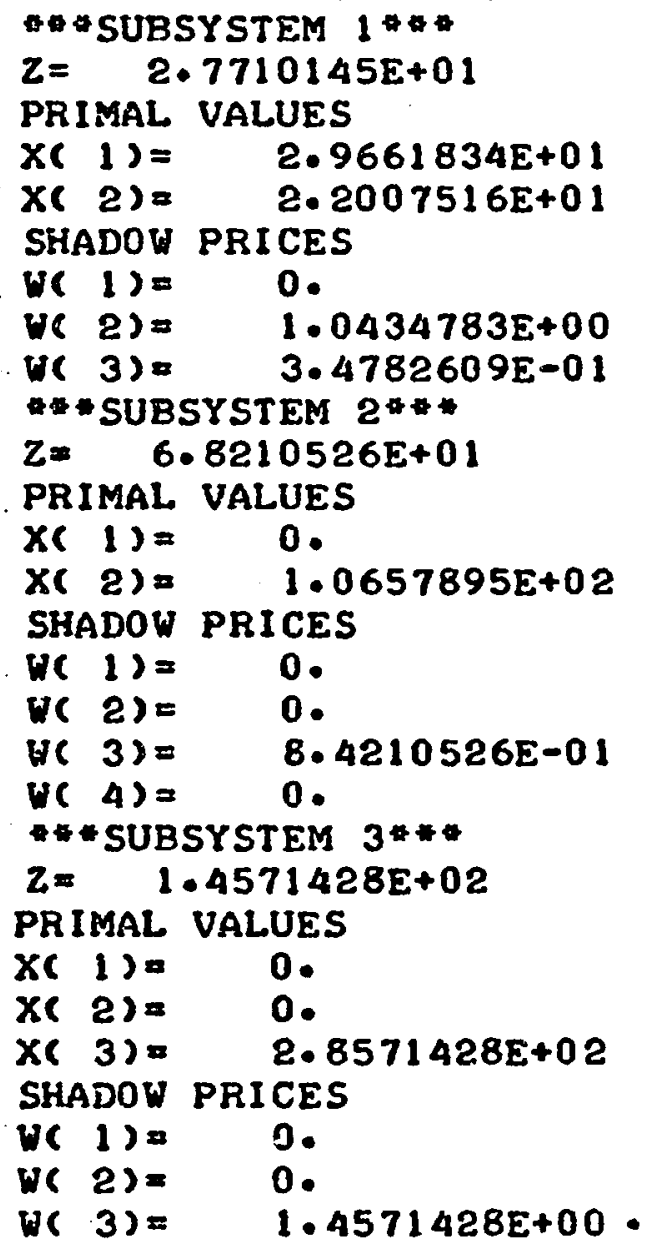




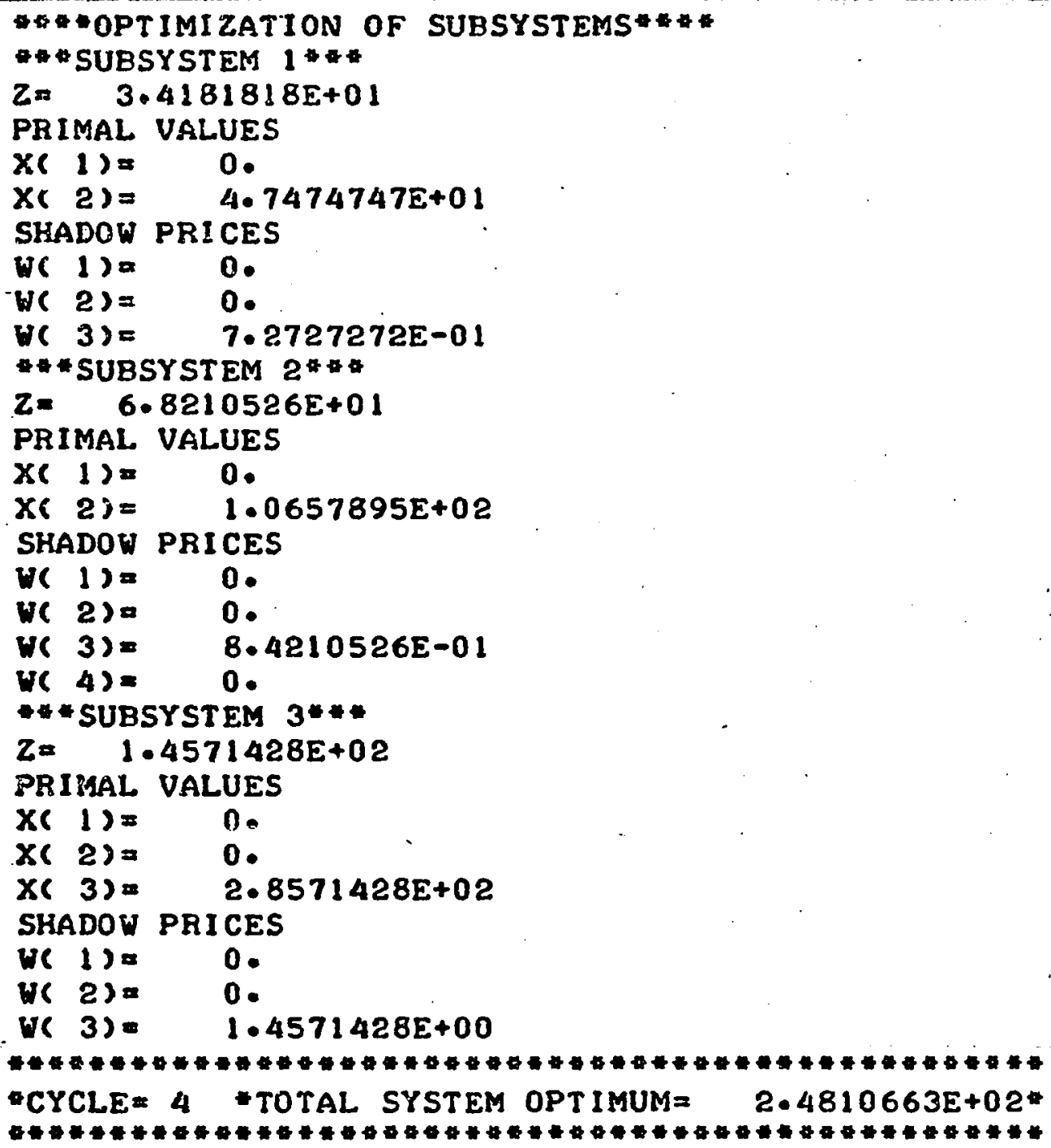


APPENDIX B

EXPERIMENTAL COMPUTER PROGRAM 


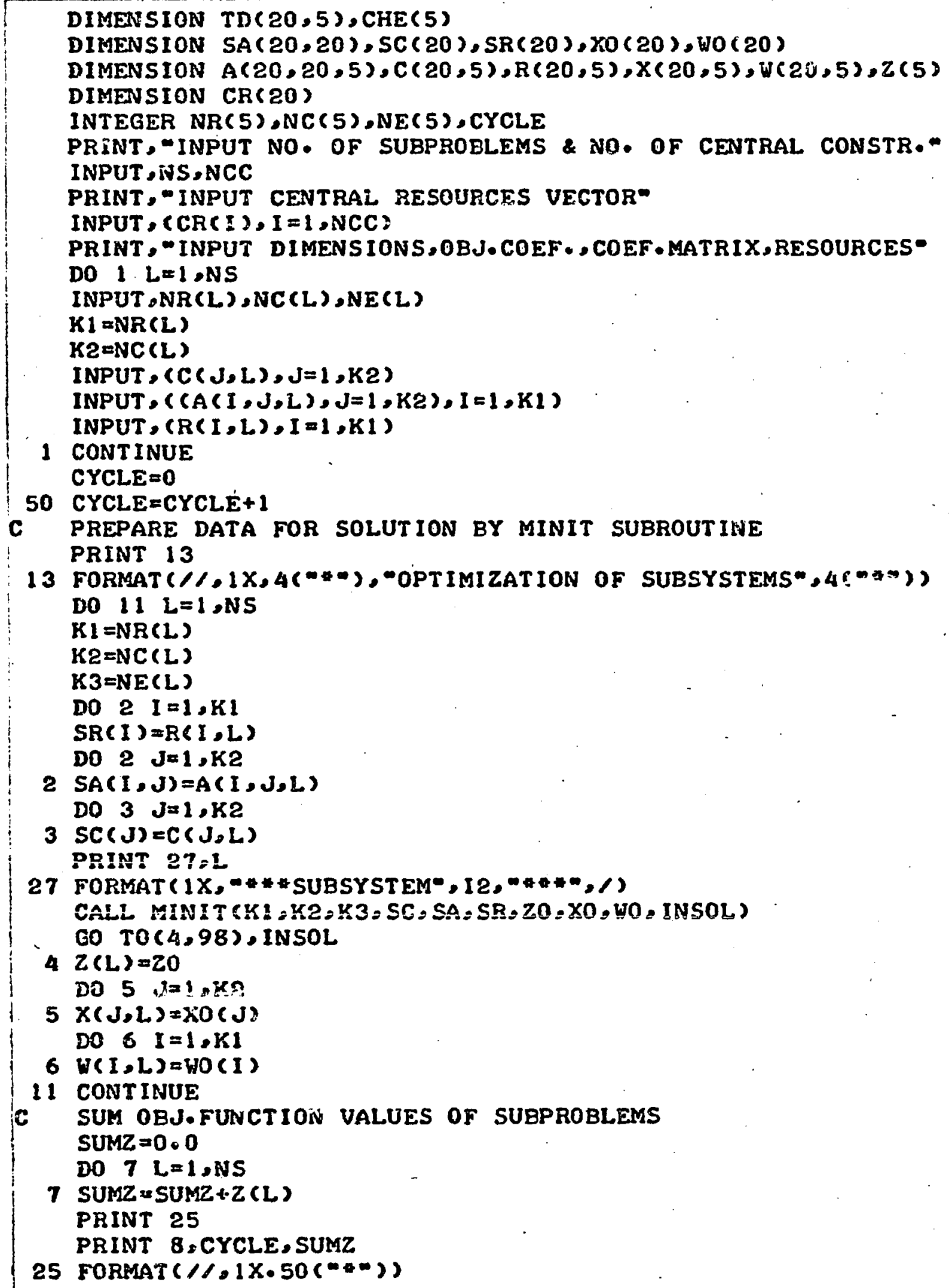




\section{FORMAT $(1 X, 50(-*-\infty), 1 / 2)$}

8 FORMAT $\left(1 X,{ }^{\circ}\right.$ CYCLE $=\star, 12,2 X, \infty$ TOTAL SYSTEM OPTIMUM=॰, 1PE16.7,**) PRINT 26

C THROUGH SUBPROBLEM SHADOW PRICES CALCULATE CHANGES IN

C ALLOCATION OF CENTRAL RESOURCES

DO $221=1$,NCC

C SUM SUBPROBLEM SHADOW PRICES OF SAME COMMODITY

i SUMW $=0.0$

DO $9 \mathrm{~L}=1$, NS

$9 S U M W=S U M W+W(I, L)$

C IF SHADOW PRICES SUM IS ZERO NO REALLOCATION IS WARRANTED IF ( SUMU) $53,55,53$

55 CONTINUE

DO $57 \mathrm{~L}=1$, NS

$57 \operatorname{TD}(I, L)=0=0$

GO TO 22

53 CONTINUE

TNS $=$ NS

DO $59 \mathrm{~L}=1, \mathrm{NS}$

$T D(I, L)=(C R(I) / S U A W) * W(I, L)-(C R(I) / T N S)$

$T D(I, L)=T D(I, L) / T N S$

$59 R(I, L)=R(I, L)+T D(I, L)$

2D CONTINUE

PRINT 78

DO $15 \mathrm{~L}=1$,NS

PRINT 27,L

DO $79 I=1$ sNCC

PRINT 66,I,L,TD(I,L)

66 FORMAT $\left(1 X,-{ }^{\circ} D(*, 12, *, \infty, 12,)^{\circ}=*, 1 P E 16,7\right)$

79 CONTINUE

18 FORMAT (1X, -ALLOCATION CHANGES**)

19 FORMAT $(2 X, \cdots R(*, 12, *,-, 12, \infty)=*, 1 P E 16.7)$

DO $17 I=1, N C C$

PRINT 19,I,L,R(I,L)

17 CONTINUE

15 CONTINUE

C CHECK OPT IMALITY

NCCl $=\mathrm{NCC}+1$

$T S U M=0.0$

DO $312=1$, NS

$K R=N C(L)$

DO 31 I $=\mathrm{NCC} 1, \mathrm{K2}$

$31 T S U M=T S U M+R(I, L) * W(I, L)$

DO $33 \mathrm{~L}=1, \mathrm{NS}$

CSUM $=0.0$

DO $35 I=1$, NCC

35 CSUM $=C S U M+C R(I) * U(I, L)$

SSS $=$ SUMZ - T SUM - CSUM

IF(SSS) $33,67,33$

33 CONT INUE 


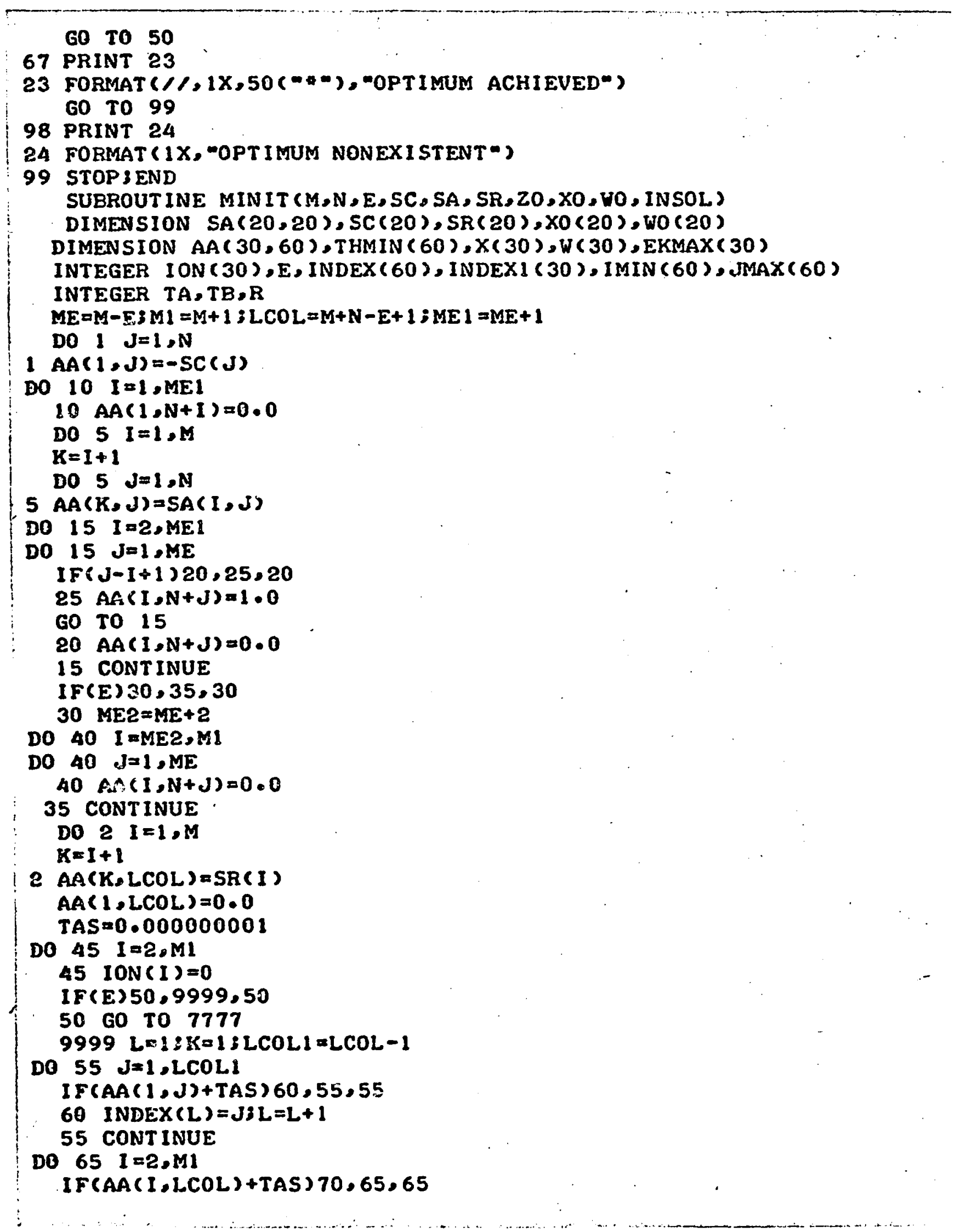


70 INDEXI $(K)=I 3 K=K+1$

65 CONT INUE

IF $(L-1) 75,80,75$

80 CONTINUE

IF $(K-1) 85,99,85$

85 CONT INUE

IF $(K-2) 9991,90,9991$

90 CONT INUE

DO $95 \mathrm{~J}=1, \mathrm{LCOL} 1$

$J J J=I N D E X I(1)$

IF (AA (JJJ,J)) $9991,95,95$

95 CONT INUE

GO TO 98

75 CONT INUE

IF $(L-2) 3,6,3$

6 CONTINUE

IF $(K-1) 12,9,12$

9 COATI INSE

BO $16 I=2, M 1$

II I INDEX ( I)

IF(AA (I, III) ) 16,16,9992

16 CONTINUE

GO TO 97

12 GO TO 9993

3 CONTINUE

IF $(K-1) 9993,9992,9993$

$9991 \pi N=1360$ TO 8888

351 TA=IMAX; TB $=$ MM GO T0 6666

9992 JAUG $=1 ;$ GO TO 5555

352 TA=IM; TB $=$ JMIN 3 GO TO 6666

9993 JAUG $=2$; 60 TO 5555

$353 \pi N=23$ GO TO 8888

354 CONTINUE

IF (RMIN-(10*6) $17,18,17$

18 TA=IMAX \$ TB=JM3 GO TO 6666

17 CONT INUE

IF(PMAX+(10*6))19.21.19

21 TA=IM3 TB=JMIN; GO TO 6666

19 CONT INUE

$1 F(A B S(P M A X)-A B S(R M I N)) 22.22 .23$

22 TA=IM; TB $=$ JMIN: GO TO 6666

23 TA=IMAX3 TB=JM: GO TO 6666

91 PRINT, "NO SOLUTION *

GO TO 1111

97 PRINT, -PRIMAL OBJECTIVE FUNCTION IS UNBOUNDED*

PRINT, "DUAL PROBLEM HAS NO FEASIBLE SOLUTIONS" GO TO 1111

98 PRINT, -PRIMAL PROBLEM HAS NO FEASIBLE SOLUTION" PRINT, "DUAL OBJECTIVE FUNCTION IS UNBOUNDED" GO TO 1111 


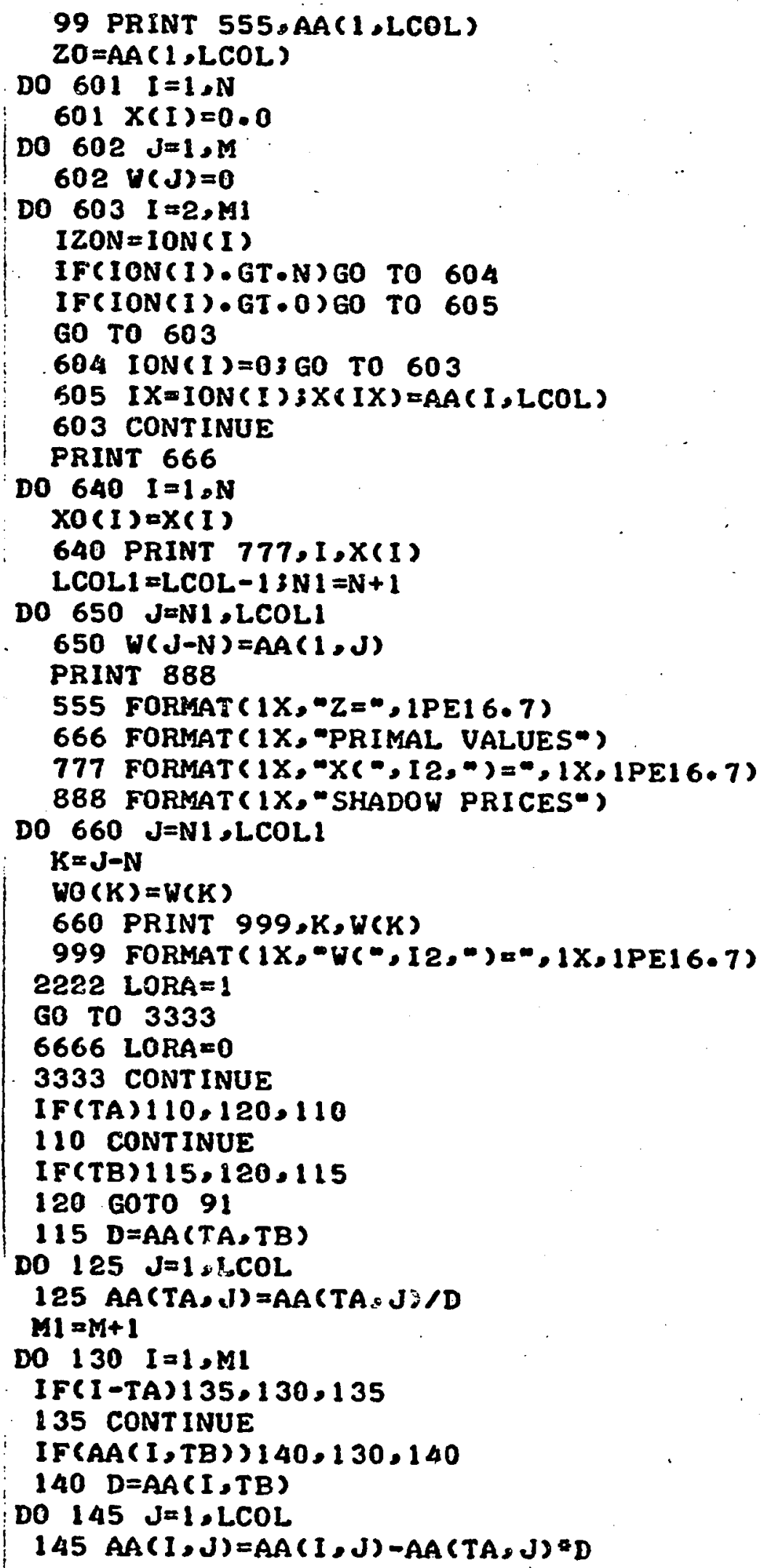




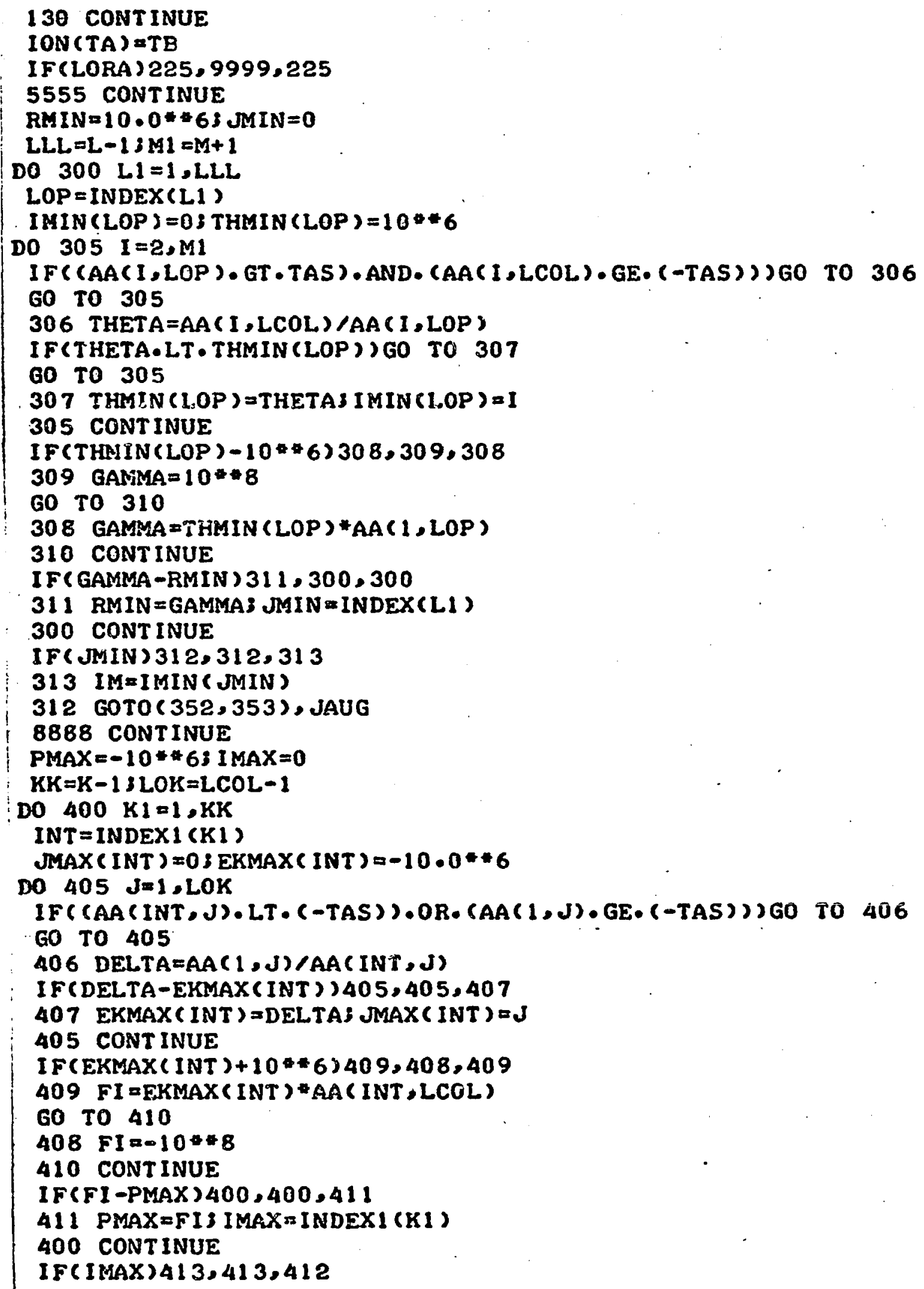




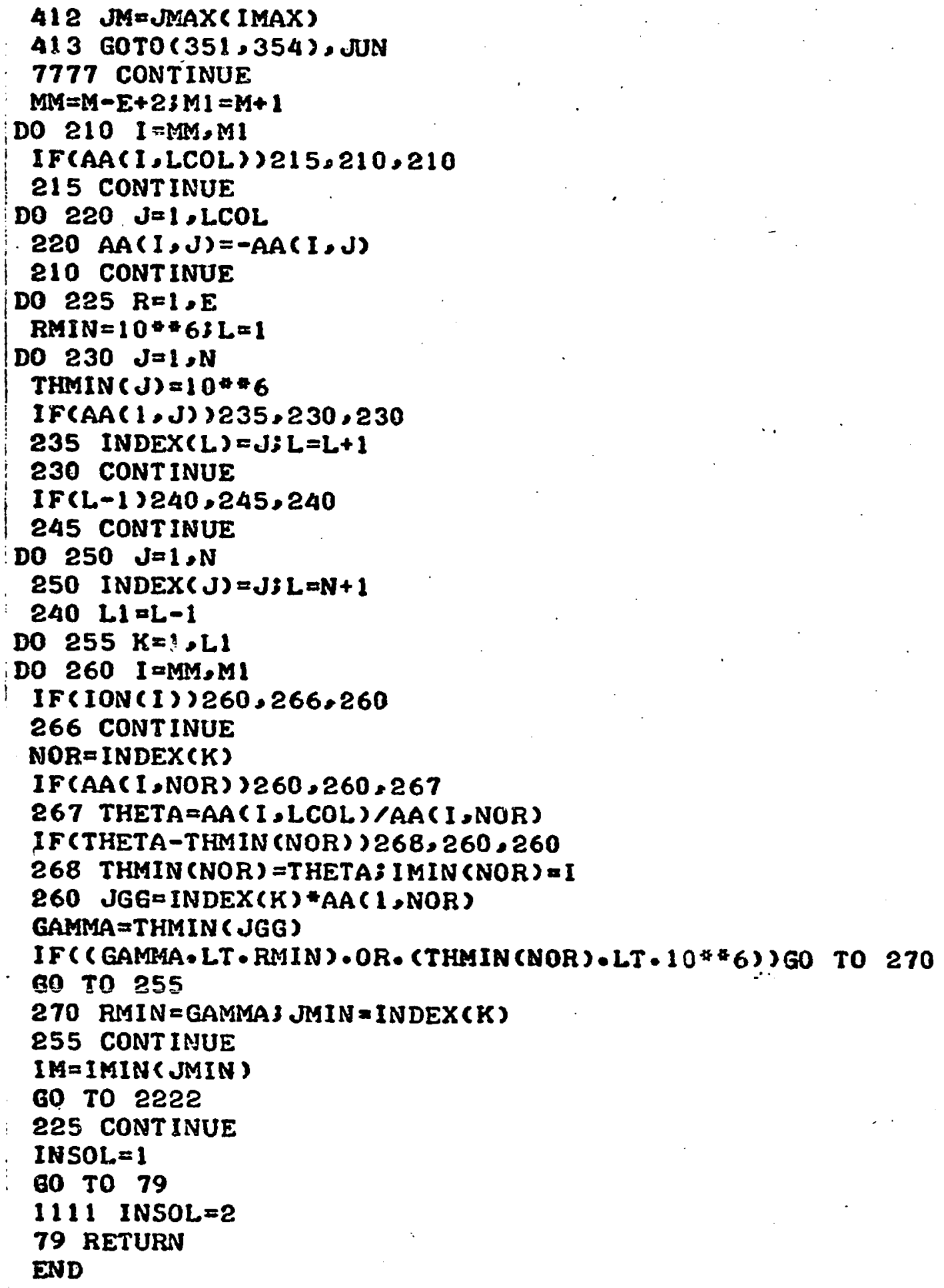

